\title{
General coorbit space theory for quasi-Banach spaces and inhomogeneous function spaces with variable smoothness and integrability
}

\author{
Henning Kempka ${ }^{1, *}$, Martin Schäfer ${ }^{2}$, Tino Ullrich $^{3}$ \\ ${ }^{1}$ Faculty of Mathematics, Technical University Chemnitz \\ Reichenhainer Straße 39, 09126 Chemnitz, Germany \\ ${ }^{2}$ Mathematical Institute, Technical University of Berlin \\ Straße des 17. Juni 136, 10623 Berlin, Germany \\ ${ }^{3}$ Hausdorff Center for Mathematics \& Institute for Numerical Simulation \\ Endenicher Allee 60, 53115 Bonn, Germany
}

October 2, 2018

\begin{abstract}
In this paper we propose a general coorbit space theory suitable to define coorbits of quasi-Banach spaces using an abstract continuous frame, indexed by a locally compact Hausdorff space, and an associated generalized voice transform. The proposed theory realizes a further step in the development of a universal abstract theory towards various function spaces and their atomic decompositions which has been initiated by Feichtinger and Gröchenig in the late 1980ies. We combine the recent approaches in Rauhut, Ullrich [50] and Rauhut 48 to identify, in particular, various inhomogeneous (quasi-Banach) spaces of Besov-Lizorkin-Triebel type. To prove the potential of our new theory we apply it to spaces with variable smoothness and integrability which have attracted significant interest in the last 10 years. From the abstract discretization machinery we obtain atomic decompositions as well as wavelet frame isomorphisms for these spaces.
\end{abstract}

Key Words: Coorbit space theory, continuous wavelet transform, Besov-Lizorkin-Triebel type spaces, variable smoothness, variable integrability, 2-microlocal spaces, Peetre maximal function, atomic decomposition, wavelet bases

AMS Subject classification: 42B25, 42B35, 46E35, 46F05.

\section{Introduction}

The birth of coorbit theory dates back to the 1980ies, starting with a series of papers by Feichtinger and Gröchenig [21, 31, 32. The main intention was to characterize function spaces

\footnotetext{
*Corresponding author, Email: henning.kempka@mathematik.tu-chemnitz.de
} 
via an abstract transform, the so-called voice transform. In the original setup, this transform is determined by an integrable irreducible representation of a locally compact group on a Hilbert space $\mathcal{H}$ unifying e.g. the continuous wavelet transform, the short-time Fourier transform, and the recent shearlet transform, to mention just a few. More recently, representations which are not necessarily irreducible nor integrable have been considered [12]. They allow to treat, for instance, Paley-Wiener spaces and spaces related to Shannon wavelets and Schrödingerlets.

Classical examples of coorbit spaces associated to the continuous wavelet transform on the $a x+b$-group are the homogeneous Besov-Lizorkin-Triebel spaces [55, 56, 57, identified rigorously as coorbits in Ullrich [59. What concerns further extensions of these spaces and interpretations as coorbits we refer to Liang et al. [42, 43]. More general wavelet coorbit spaces associated to a semidirect product $G=\mathbb{R}^{d} \rtimes H$, with a suitable subgroup $H$ of $G L\left(\mathbb{R}^{d}\right)$ as dilation group, have been studied in [27, 28, 29] and could recently be identified with certain decomposition spaces on the Fourier domain [30]. A specific example of this general setup is the shearlet transform, where $G$ is the shearlet group. The associated shearlet spaces have first been studied in [9]. Other coorbit spaces, based on a voice transform different from the wavelet transform, are e.g. modulation spaces [33, 19] and Bergman spaces [21].

Coorbit theory thus covers a great variety of different function spaces. The underlying group structure however turns out to be a severe restriction for the theory since the identification of, e.g., inhomogeneous spaces of the above type was long time not possible, however desirable. For that reason the theory has evolved and several subsequent contributions have weakened among others the assumption that the voice transform is supported on a locally compact group. For instance, Dahlke, Steidl, and Teschke replaced it by a homogeneous space, i.e., a quotient of a group with a subgroup, with the aim to treat functions on manifolds [10, 11, 8].

The starting point for the general coorbit space theory presented in this paper is the approach used by Fornasier and Rauhut [24, which was later revised and extended in [25] and further expanded in [50]. There, the group structure is abandoned completely and the voice transform is determined solely by an abstract continuous frame $\mathcal{F}=\left\{\varphi_{x}\right\}_{x \in X}$ in $\mathcal{H}$ indexed by a locally compact Hausdorff space $X$ (not necessarily a group), i.e., $X$ is equipped with a Radon measure $\mu$ such that the map $x \mapsto \varphi_{x}$ is weakly measurable and that with constants $0<C_{1}, C_{2}<\infty$

$$
C_{1}\left\|f\left|\mathcal{H}\left\|^{2} \leqslant \int_{X}\left|\left\langle f, \varphi_{x}\right\rangle\right|^{2} d \mu(x) \leqslant C_{2}\right\| f\right| \mathcal{H}\right\|^{2} \quad \text { for all } f \in \mathcal{H} .
$$

(Note that weak measurability of $x \mapsto \varphi_{x}$ in $\mathcal{H}$ implies that the integral in (1.1) is well-defined.)

We combine the approach in [50] with ideas from [48] to define even coorbits

$$
\operatorname{Co}(\mathcal{F}, Y):=\left\{f:\left\langle f, \varphi_{x}\right\rangle \in Y\right\}
$$

of quasi-Banach spaces $Y$ using the general voice transform associated to $\mathcal{F}$. We thereby also recall the relevant details of the existing theory, especially from [24, 50] and fix some earlier inaccuracies. The developed theory yields noteworthy generalizations even for the Banach case, e.g. some assumptions made in [24, 50] can be weakened, such as the uniform boundedness of the analyzing frame $\mathcal{F}$ or some technical restrictions on the weights and the admissible coverings. Most notably however, we can generalize the main results of the discretization theory, which is possible since we take a different - more direct - route to establish them. It turns out that the three essential Lemmas 2.36, 2.40, and 2.47 below constitute the technical foundation for the proof of the general abstract discretization results in Theorems 2.48 and 2.50, 
Putting these lemmas at the center of the exposition simplifies many arguments and allows for a systematic approach towards new abstract discretization results. In fact, we obtain discrete characterizations of coorbit spaces by "sampling" the function using a sub-sampled discrete frame $\mathcal{F}_{d}=\left\{\varphi_{x_{i}}\right\}_{i \in I}$ on a suitable index set $I$. Of course, as usual in coorbit space theory, there are several technical assumptions to check. However, a great advantage of the presented discretization machinery is the fact that it provides a straight path towards discretization, where matters essentially reduce to checking properties (associated to $Y$ ) of the analyzing frame $\mathcal{F}$. This is in contrast to the usual approach where atomic decompositions and wavelet characterizations, useful to study embeddings, $s$-numbers, interpolation properties etc., are often developed from scratch for different related function spaces.

To prove the potential of the theory presented here, we apply it to identify spaces with variable smoothness and integrability, so-called variable exponent spaces, as coorbits. TriebelLizorkin spaces of this kind are defined via the quasi-norm

$$
\left\|f\left|F_{p(\cdot), q(\cdot)}^{w}\left(\mathbb{R}^{d}\right)\|=\|\left(\sum_{j=0}^{\infty}\left|w_{j}(\cdot)\left(\Phi_{j} * f\right)(\cdot)\right|^{q(\cdot)}\right)^{1 / q(\cdot)}\right| L_{p(\cdot)}\left(\mathbb{R}^{d}\right)\right\|,
$$

where the functions $w_{j}$ are weights and $\Phi_{j}$ are frequency filters corresponding to a dyadic decomposition of the frequency plane. For the precise formulation see Definition 3.8 below. The functions $p(\cdot), q(\cdot)$ represent certain integrability parameters, which may vary in the spatial variable $x$ of the space. The 2 -microlocal weight sequence $w_{j}(\cdot)$ determines the variable smoothness, see [39] for details. Function spaces with variable exponents are a fast developing field thanks to its many applications in stochastics, fluid dynamics and image processing, see [17] and [16] and references therein. The Lebesgue spaces $L_{p(\cdot)}\left(\mathbb{R}^{d}\right)$ with variable integrability, see Definition 3.1 below, were already used by Orlicz [46. Recent contributions by Diening [14] on the boundedness of the Hardy-Littlewood maximal operator on $L_{p(\cdot)}\left(\mathbb{R}^{d}\right)$ make them accessible for harmonic analysis issues.

Surprisingly, the spaces (1.2) can be handled within the generalized coorbit space theory presented in this paper. In fact, due to unbounded left and right translation operators (within the $a x+b$-group) a coorbit characterization of homogeneous spaces of the above type already seems to be rather impossible at first glance. However, we are able to identify them as coorbits $\mathrm{Co}(\mathcal{F}, Y)$ of, what we call, Peetre-Wiener type spaces $Y$ by using a suitable continuous frame $\mathcal{F}=\left\{\varphi_{x}\right\}_{x \in X}$ with the index set $X=\mathbb{R}^{d} \times[(0,1) \cup\{\infty\}]$. These spaces $Y$ are solid quasiBanach function spaces (QBF) defined on $X$, see Section 4.1 below. Peetre-Wiener type spaces can be seen as a mixture of the Peetre type spaces introduced in [59], and certain Wiener amalgam spaces, see [22, [49]. They appear naturally when dealing with continuous local mean characterizations, a strategy developed in [59] and [42]. In fact, we show in Subsection 3.3 below that with large enough $a>0$ the quantity

$$
\begin{aligned}
\left\|f \mid F_{p(\cdot), q(\cdot)}^{w}\left(\mathbb{R}^{d}\right)\right\|_{3} & =\left\|w(\cdot, \infty)\left\langle\Phi_{0}^{*} f\right\rangle_{a}(\cdot) \mid L_{p(\cdot)}\left(\mathbb{R}^{d}\right)\right\| \\
& +\left\|\left(\int_{0}^{1}\left|w(\cdot, t)\left\langle\Phi_{t}^{*} f\right\rangle_{a}(\cdot)\right|^{q(\cdot)} \frac{d t}{t}\right)^{1 / q(\cdot)} \mid L_{p(\cdot)}\left(\mathbb{R}^{d}\right)\right\|
\end{aligned}
$$

represents an equivalent characterization for $F_{p(\cdot), q(\cdot)}^{w}\left(\mathbb{R}^{d}\right)$. Here

$$
\left\langle\Phi_{t}^{*} f\right\rangle_{a}(x):=\sup _{\substack{z \in \mathbb{R}^{d} \\ t / 2 \leqslant \tau \leqslant 2 t, \tau<1}} \frac{\left|\left(\Phi_{\tau} * f\right)(x+z)\right|}{(1+|z| / \tau)^{a}}
$$


denotes the corresponding maximal function, which is essentially a modification of the widely used Peetre maximal function, see (3.7) below, and is used in the definition of the Peetre-Wiener type spaces, see Definition 4.2. Now the representation (1.3) is actually the identification of

$F_{p(\cdot), q(\cdot)}^{w}\left(\mathbb{R}^{d}\right)$ as a coorbit space of a Peetre-Wiener type space. Applying the abstract theory, in particular Theorem 2.50, we obtain biorthogonal wavelet expansions [6] of the respective coorbit spaces. We describe the application of the machinery for the rather simple (orthogonal) Meyer wavelets, see Appendix A.2. Due to its generality, a straightforward modification of Theorem 2.50 leads to general (biorthogonal) wavelet expansions and other tight discrete wavelet frames.

Let us mention that the continuous local mean characterizations (1.3) of spaces with variable exponents, see also Theorem 3.11, are new and interesting for their own sake. In fact, one has to deal with additional difficulties since a version of the classical Fefferman-Stein maximal inequality, a crucial tool in this respect, is in general not true in $L_{p(\cdot)}\left(\ell_{q(\cdot)}\right)$ if $q(\cdot)$ is nonconstant.

Finally, the provided discretizations of such spaces are not entirely new. In [37] the author used a different technique in order to obtain discretizations with Meyer and Daubechies wavelets. However, let us mention that the abstract Theorems 2.48, 2.50 below neither restrict to orthonormal wavelets nor compactly supported atoms.

\subsection{Outline}

The paper is structured as follows. The abstract theory is established in Section 2, It generalizes earlier contributions, especially [24, 50], and in particular now includes the quasi-Banach case. In Section 3 we give a short introduction to variable exponent spaces, which will serve as our demonstration object for a concrete application of the theory. We will utilize a new continuous local means characterization in Section 4 to identify them as coorbits of a new scale of PeetreWiener type spaces. The abstract theory then yields atomic decompositions as well as discrete characterizations via wavelet frames. Some useful facts concerning the continuous and discrete (orthogonal) wavelet transform are collected in the Appendix.

\subsection{Notation}

The symbols $\mathbb{N}, \mathbb{N}_{0}, \mathbb{Z}, \mathbb{R}, \mathbb{R}_{+}$, and $\mathbb{C}$ denote the natural numbers, the natural numbers including 0 , the integers, the real numbers, the non-negative real numbers, and the complex numbers. For a real number $t \in \mathbb{R}$ we put $(t)_{+}=\max \{t, 0\}$ and $(t)_{-}=\min \{t, 0\}$. The conjugation of $z \in \mathbb{C}$ is denoted by $\bar{z}$. Let us emphasize that $\mathbb{R}^{d}$ has the usual meaning and $d \in \mathbb{N}_{0}$ is reserved for its dimension. The symbol $|\cdot|$ denotes the Euclidean norm on $\mathbb{R}^{d}$ and $|\cdot|_{1}$ the $\ell_{1}$-norm.

The space of all sequences with entries in some set $M$ over some countable index set $I$ is denoted by $M^{I}$ and we write $\Lambda(i)$ for the $i$-th sequence element of a sequence $\Lambda \in M^{I}$.

For topological vector spaces $Y$ and $Z$ the class of linear continuous mappings from $Y$ to $Z$ is denoted by $\mathcal{L}(Y, Z)$. The notation $\Phi: Y \hookrightarrow Z$ indicates that $Y$ is continuously embedded into $Z$, i.e., $\Phi$ is an injective continuous linear map from $Y$ into $Z$. If the embedding is canonical we simply write $Y \hookrightarrow Z$. If $Y$ is equipped with a quasi-norm we use $\|f \mid Y\|$ for the quasi-norm of $f \in Y$. The operator quasi-norm of $A \in \mathcal{L}(Y, Z)$ is denoted by $\|A \mid Y \rightarrow Z\|$.

We use the notation $a \lesssim b$ if there exists a constant $c>0$ (independent of the context dependent relevant parameters) such that $a \leqslant c b$. If $a \lesssim b$ and $b \lesssim a$ we write $a \asymp b$. Furthermore, we write $Y \asymp Z$ for two quasi-normed spaces $Y, Z$ which coincide as sets and whose quasi-norms are equivalent. 


\section{General coorbit space theory}

Let $\mathcal{H}$ be a separable Hilbert space and $X$ a locally compact Hausdorff space endowed with a positive Radon measure $\mu$ with $\operatorname{supp} \mu=X$. A family $\mathcal{F}=\left\{\varphi_{x}\right\}_{x \in X}$ of vectors in $\mathcal{H}$ is called a continuous frame (see [1]) if the assignment $x \mapsto \varphi_{x}$ is weakly measurable and if there exist constants $0<C_{1}, C_{2}<\infty$ such that (1.1) is satisfied. Let us record an important property.

Lemma 2.1. Let $\mathcal{F}=\left\{\varphi_{x}\right\}_{x \in X}$ be a continuous frame in $\mathcal{H}$ and $N \subset X$ a set of measure zero. Then $\left\{\varphi_{x}\right\}_{x \in X \backslash N}$ is total in $\mathcal{H}$.

Proof. Let us put $X^{*}:=X \backslash N$. We have to show that $V:=\operatorname{span}\left\{\varphi_{x}: x \in X^{*}\right\}$ is dense in $\mathcal{H}$. Indeed, using the frame property of $\mathcal{F}$, we can deduce for every $f \perp V$

$$
\left\|\left.f\left|\mathcal{H} \|^{2}=\int_{X}\right|\left\langle f, \varphi_{x}\right\rangle\right|^{2} d \mu(x)=\int_{X^{*}}\left|\left\langle f, \varphi_{x}\right\rangle\right|^{2} d \mu(x)=0 .\right.
$$

To avoid technicalities, we assume throughout this paper that $X$ is $\sigma$-compact. We further assume that the continuous frame is Parseval, i.e. $C_{1}=C_{2}=1$, and note that - apart from minor changes - the theory presented here is valid also for general tight frames where $C_{1}=C_{2}$. It is also possible to develop the theory in the setting of non-tight frames, where the associated coorbit theory has been worked out in [24 - at least to a significant extent.

For $0<p<\infty$ we define the Lebesgue space $L_{p}(X):=L_{p}(X, \mu)$ as usual by

$$
\left\|F \mid L_{p}(X, \mu)\right\|:=\left(\int_{X}|F(x)|^{p} d \mu(x)\right)^{1 / p}<\infty .
$$

A function $F$ belongs to $L_{\infty}(X):=L_{\infty}(X, \mu)$ if and only if $F$ is essentially bounded. The corresponding sequence spaces $\ell_{p}(I)$ are obtained by choosing $X$ as a countable index set $I$, equipped with the discrete topology and counting measure $\mu$.

Associated to a continuous frame $\mathcal{F}$ is the voice transform $V_{\mathcal{F}}: \mathcal{H} \rightarrow L_{2}(X, \mu)$ defined by

$$
V_{\mathcal{F}} f(x)=\left\langle f, \varphi_{x}\right\rangle, \quad f \in \mathcal{H}, x \in X,
$$

and its adjoint $V_{\mathcal{F}}^{*}: L_{2}(X, \mu) \rightarrow \mathcal{H}$ given in a weak sense by the integral

$$
V_{\mathcal{F}}^{*} F=\int_{X} F(y) \varphi_{y} d \mu(y)
$$

Since we assume the frame $\mathcal{F}$ to be Parseval $V_{\mathcal{F}}$ is an isometry and in particular injective. The adjoint $V_{\mathcal{F}}^{*}$ is surjective with $\left\|V_{\mathcal{F}}^{*} \mid L_{2} \rightarrow \mathcal{H}\right\|=1$ and the associated frame operator $S_{\mathcal{F}}:=$ $V_{\mathcal{F}}^{*} V_{\mathcal{F}}$ is the identity. Hence we have

$$
f=\int_{X} V_{\mathcal{F}} f(y) \varphi_{y} d \mu(y) \quad \text { and } \quad V_{\mathcal{F}} f(x)=\int_{X} V_{\mathcal{F}} f(y)\left\langle\varphi_{y}, \varphi_{x}\right\rangle d \mu(y) .
$$

The second identity is the crucial reproducing formula $R_{\mathcal{F}}\left(V_{\mathcal{F}} f\right)=V_{\mathcal{F}} f$ for $f \in \mathcal{H}$, where

$$
R_{\mathcal{F}}(x, y)=\left\langle\varphi_{y}, \varphi_{x}\right\rangle, \quad x, y \in X,
$$

is an integral kernel (operator), referred to as the frame kernel associated to $\mathcal{F}$. It acts as a selfadjoint bounded operator $R_{\mathcal{F}}=V_{\mathcal{F}} V_{\mathcal{F}}^{*}: L_{2}(X) \rightarrow L_{2}(X)$, which is an orthogonal projection 
with $R_{\mathcal{F}}\left(L_{2}(X)\right)=V_{\mathcal{F}}(\mathcal{H})$. The converse of the reproducing formula is also true, i.e., if $F \in L_{2}(X)$ satisfies $R_{\mathcal{F}}(F)=F$ then there exists a unique element $f \in \mathcal{H}$ such that $V_{\mathcal{F}} f=F$.

We remark that we use the same notation for the function $R_{\mathcal{F}}: X \times X \rightarrow \mathbb{C}$ given in (2.2) and the associated operator $R_{\mathcal{F}}: L_{2}(X) \rightarrow L_{2}(X)$. It is important to note that the function $R_{\mathcal{F}}$ is measurable. Indeed, utilizing an orthonormal basis $\left(f_{n}\right)_{n \in \mathbb{N}}$ of $\mathcal{H}$ we can expand $R_{\mathcal{F}}(x, y)=\sum_{n \in \mathbb{N}}\left\langle\varphi_{y}, f_{n}\right\rangle\left\langle f_{n}, \varphi_{x}\right\rangle$ as a point-wise limit of measurable functions.

The idea of coorbit theory is to measure "smoothness" of $f$ via properties of the transform $V_{\mathcal{F}} f$. Loosely speaking, the coorbit of a function space on $X$ is its retract with respect to (a suitably extended version of) the voice transform. The classical theory and its generalizations have been developed for the case of certain Banach function spaces on $X$. In the classical setup, where $X$ is equipped with a group structure, the extension [48, deals with the quasi-Banach case, and our aim is to extend the generalized theory from [24, 50] analogously.

\subsection{Function spaces on $X$}

We consider (quasi)-Banach function spaces, or shortly (Q)BF-spaces, which are linear spaces of measurable functions on $X$, equipped with a quasi-norm under which they are complete. Hereby, functions are identified when equal almost everywhere. Hence, when speaking of a function one often actually refers to an equivalence class. In general, this inaccuracy of language does not pose a problem. Only when it comes to point evaluations the precise meaning must be made clear in the context.

Recall that a quasi-norm on a linear space $Y$ generalizes the concept of a norm by replacing the triangle inequality with the more general quasi-triangle inequality

$$
\|f+g\| \leqslant C_{Y}(\|f\|+\|g\|), \quad f, g \in Y
$$

with associated quasi-norm constant $C_{Y} \geqslant 1$. Many aspects of the theory of normed spaces carry over to the quasi-norm setting, e.g. boundedness and continuity coincide, all $d$-dimensional quasi-norms are equivalent, etc.. An important exception is the Hahn-Banach theory concerned with the dual spaces. Note that the (topological) dual $Y^{\prime}$ of a quasi-normed space $Y$, equipped with the usual operator norm, is always a Banach space. Due to the possible non-convexity of the quasi-norm however, it may not be sufficiently large for the Hahn-Banach theorems to hold. In fact, $Y^{\prime}$ may even be trivial as the example of the $L_{p}$-spaces in the range $0<p<1$ shows. This fact poses a serious problem for the theory.

An important tool for dealing with quasi-norms is the Aoki-Rolewicz theorem [3, 51, which states that in every quasi-normed space $Y$ there exists an equivalent $r$-norm - in the sense of an equal topology - where an $r$-norm, $0<r \leqslant 1$, satisfies the $r$-triangle inequality

$$
\|f+g\|^{r} \leqslant\|f\|^{r}+\|g\|^{r}, \quad f, g \in Y,
$$

and in particular is a quasi-norm with constant $C_{Y}=2^{1 / r-1}$. The exponent $r=1 /\left(\log _{2} C_{Y}+1\right)$ of the equivalent $r$-norm is called the exponent of $Y$.

For a viable theory we need to further restrict the class of function spaces. A quasi-normed function space $Y$ on $X$ is called solid, if the following condition is valid,

$$
f \mu \text {-measurable, } g \in Y,|f(x)| \leqslant|g(x)| \text { a.e. } \quad \Rightarrow \quad f \in Y \text { and }\|f|Y\|\leqslant\| g| Y\| \text {. }
$$


In a solid space $Y$ we have the equality $\||f||Y\|=\| f| Y\|$ for every $f \in Y$. Moreover, there is a useful criterion for a function $f$ to belong to $Y$,

$$
f \in Y \quad \Leftrightarrow \quad|f| \in Y \text { and } f \mu \text {-measurable. }
$$

A function space shall be called rich, if it contains the characteristic functions $\chi_{K}$ for all compact subsets $K \subset X$. A rich solid quasi-normed function space on $X$ then contains the characteristic functions $\chi_{U}$ for all relatively compact, measurable subsets $U \subset X$.

We will subsequently develop coorbit theory mainly for rich solid QBF-spaces $Y$, that are continuously embedded into $L_{1}^{\text {loc }}(X)$. As usual, the spaces $L_{p}^{\text {loc }}(X):=L_{p}^{\text {loc }}(X, \mu), 0<p \leqslant \infty$, consist of all functions $F$ where $\left\|F \chi_{K} \mid L_{p}(X)\right\|<\infty$ for every compact subset $K \subset X$. The case, where $Y \nrightarrow L_{1}^{\text {loc }}(X)$, is shortly commented on at the end of Subsection 2.4

It is important to understand the relation between the quasi-norm convergence and the pointwise convergence of a sequence of functions in $Y$. We have the following result.

Lemma 2.2. Let $Y$ be a solid quasi-normed function space on $X$, and assume $f_{n} \rightarrow f$ in $Y$. Then for arbitrary but fixed representing functions $\tilde{f}_{n}, \tilde{f}$ the following holds true. For a.e. $x \in X$ there is a subsequence $\left(f_{n_{k}}\right)_{k \in \mathbb{N}}$, whose choice may depend on the particular $x \in X$, such that $\tilde{f}_{n_{k}}(x) \rightarrow \tilde{f}(x)$ as $k \rightarrow \infty$.

Proof. Assume first that $f_{n} \rightarrow 0$ in the quasi-norm of $Y$, which implies $\left\|f_{n} \mid Y\right\| \rightarrow 0$. As $\inf _{m \geqslant n}\left|f_{m}\right|$ is a measurable function with $\inf _{m \geqslant n}\left|f_{m}\right| \leqslant\left|f_{k}\right|$ for all $k \geqslant n$ we have $\inf _{m \geqslant n}\left|f_{m}\right| \in$ $Y$ with $\left\|\inf _{m \geqslant n}\left|f_{m}\right|\left|Y\|\leqslant\| f_{k}\right| Y\right\|$ for all $k \geqslant n$ by solidity. It follows $0 \leqslant\left\|\inf _{m \geqslant n}\left|f_{m}\right| \mid Y\right\| \leqslant$ $\inf _{m \geqslant n}\left\|f_{m} \mid Y\right\|=0$, and hence $\inf _{m \geqslant n}\left|\tilde{f}_{m}\right|(x)=0$ for a.e. $x \in X$. This implies that for these $x \in X$ there is a subsequence $\left(f_{n_{k}}\right)_{k \in \mathbb{N}}$ such that $\tilde{f}_{n_{k}}(x) \rightarrow 0$. Now let $f_{n} \rightarrow f$. Then $\left(f_{n}-f\right) \rightarrow 0$ and by the previous argumentation for a.e. $x \in X$ there is a subsequence $\left(f_{n_{k}}\right)_{k \in \mathbb{N}}$ such that $\tilde{f}_{n_{k}}(x)-\tilde{f}(x) \rightarrow 0$, whence $\tilde{f}_{n_{k}}(x) \rightarrow \tilde{f}(x)$.

Remark 2.3. A more thorough investigation of pointwise convergence in solid quasi-normed function spaces is carried out in [54]. It turns out that Lemma 2.2 can be strengthened using 154. Cor. 2.2.9] and the fact that $X$ is $\sigma$-finite (see Step 1 in the proof of Lemma 2.14). In fact, there is a subsequence $\left(f_{n_{k}}\right)_{k \in \mathbb{N}}$, independent of $x \in X$, with $\tilde{f}_{n_{k}}(x) \rightarrow \widetilde{f}(x)$ for a.e. $x \in X$.

\section{$2.2 \quad$ Associated sequence spaces}

Let us take a look at sequence spaces associated with a function space $Y$ on $X$. For this we recall the notion of an admissible covering introduced in [24, 50. We say that a covering $\mathcal{U}=\left\{U_{i}\right\}_{i \in I}$ of $X$ is locally finite if every $x \in X$ possesses a neighborhood which intersects only a finite number of the covering sets $U_{i}$.

Definition 2.4. A covering $\mathcal{U}=\left\{U_{i}\right\}_{i \in I}$ of $X$ is called admissible, if it is locally finite and if it satisfies the following conditions:

(i) Each $U_{i}$ is measurable, relatively compact and has non-void interior.

(ii) The intersection number $\sigma(\mathcal{U}):=\sup _{i \in I} \sharp\left\{j: U_{i} \cap U_{j} \neq \varnothing\right\}$ is finite.

A covering of a locally compact Hausdorff space is locally finite if and only if every compact subset intersects only a finite number of the covering sets. Hence, every locally finite covering of the $\sigma$-compact space $X$ is countable. In particular, the following lemma holds true. 
Lemma 2.5. Every admissible covering of the $\sigma$-compact space $X$ has a countable index set.

Following [24, 50] we now define two types of sequence spaces associated to $Y$.

Definition 2.6. For a rich solid $Q B F$-space $Y$ on $X$ and an admissible covering $\mathcal{U}=\left\{U_{i}\right\}_{i \in I}$ of $X$ the sequence spaces $Y^{b}$ and $Y^{\natural}$ associated to $Y$ and $\mathcal{U}$ are defined by

$$
\begin{aligned}
& Y^{\natural}=Y^{\natural}(\mathcal{U}):=\left\{\left\{\lambda_{i}\right\}_{i \in I}:\left\|\left\{\lambda_{i}\right\}_{i \in I}\left|Y^{b}\|:=\| \sum_{i \in I}\right| \lambda_{i}\left|\chi_{U_{i}}\right| Y\right\|<\infty\right\}, \\
& Y^{\natural}=Y^{\natural}(\mathcal{U}):=\left\{\left\{\lambda_{i}\right\}_{i \in I}:\left\|\left\{\lambda_{i}\right\}_{i \in I}\left|Y^{\natural}\|:=\| \sum_{i \in I}\right| \lambda_{i}\left|\mu\left(U_{i}\right)^{-1} \chi_{U_{i}}\right| Y\right\|<\infty\right\} .
\end{aligned}
$$

Note that due to Lemma 2.5 the index set $I$ of these sequence spaces is necessarily countable. Also observe that due to condition (i) of Definition 2.4 and $\operatorname{supp} \mu=X$ we have $\mu\left(U_{i}\right)>0$ for every $i \in I$, and in turn $\left\|\chi_{U_{i}} \mid Y\right\|>0$.

Viewing a sequence as a function on the index set $I$, equipped with the counting measure, we subsequently use the terminology introduced above for function spaces. For better distinction, we will speak of a quasi-Banach sequence space and use the abbreviation QBS-space.

Proposition 2.7. The sequence spaces $Y^{b}(\mathcal{U})$ and $Y^{\natural}(\mathcal{U})$ are rich solid $Q B S$-spaces with the same quasi-norm constant $C_{Y}$ as $Y$.

Before we give the proof of this proposition let us establish some useful embedding results. First observe that the mapping

$$
I_{b}^{\natural}: Y^{b} \rightarrow Y^{\natural}, \lambda_{i} \mapsto \mu\left(U_{i}\right) \lambda_{i}
$$

is an isometric isomorphism between $Y^{b}$ and $Y^{\natural}$, which allows to transfer statements from one space to the other. Moreover, if $\inf _{i \in I} \mu\left(U_{i}\right)>0$ we have the embedding $Y^{b} \hookrightarrow Y^{\natural}$. Analogously, $\sup _{i \in I} \mu\left(U_{i}\right)<\infty$ implies $Y^{\natural} \hookrightarrow Y^{b}$. Consequently, $Y^{b}=Y^{\natural}$ if both conditions are fulfilled.

Let $\nu: I \rightarrow[0, \infty)$ be a discrete weight and define $\left\|\Lambda\left|\ell_{p}^{\nu}\|:=\| \Lambda \nu\right| \ell_{p}\right\|$ for $0<p \leqslant \infty$ and $\Lambda \in \mathbb{C}^{I}$. The space $\ell_{p}^{\nu}(I):=\left\{\Lambda \in \mathbb{C}^{I}:\left\|\Lambda \mid \ell_{p}^{\nu}\right\|<\infty\right\}$ is a QBS-space with quasi-norm $\left\|\cdot \mid \ell_{p}^{\nu}\right\|$.

Lemma 2.8. Let $0<p \leqslant 1$ be the exponent of $Y$. We then have the continuous embeddings

$$
\ell_{p}^{\omega^{b}}(I) \hookrightarrow Y^{b}(\mathcal{U}) \hookrightarrow \ell_{\infty}^{\omega^{b}}(I) \quad \text { and } \quad \ell_{p}^{\omega^{\natural}}(I) \hookrightarrow Y^{\natural}(\mathcal{U}) \hookrightarrow \ell_{\infty}^{\omega^{\natural}}(I)
$$

with weights defined by $\omega^{b}(i):=\left\|\chi_{U_{i}} \mid Y\right\|$ and $\omega^{\natural}(i):=\mu\left(U_{i}\right)^{-1}\left\|\chi_{U_{i}} \mid Y\right\|$ for $i \in I$.

Proof. We have $\left\|\left\{\lambda_{i}\right\}_{i \in I}\left|Y^{b}\left\|^{p}=\right\| \sum_{i \in I}\right| \lambda_{i}\left|\chi_{U_{i}}\right| Y\right\|^{p} \lesssim \sum_{i \in I}\left|\lambda_{i}\right|^{p}\left\|\chi_{U_{i}}\left|Y\left\|^{p}=\right\|\left\{\lambda_{i}\right\}_{i \in I}\right| \ell_{p}^{\omega^{b}}\right\|^{p}$ for $\left\{\lambda_{i}\right\}_{i \in I} \in \ell_{p}^{\omega^{b}}$. If $\left\{\lambda_{i}\right\}_{i \in I} \in Y^{b}$ we can estimate for every $j \in I$

$$
\left|\lambda_{j}\right| \omega^{b}(j)=\left|\lambda_{j}\right|\left\|\chi_{U_{j}}|Y\|=\|| \lambda_{j}\left|\chi_{U_{j}}\right| Y\right\| \leqslant\left\|\sum_{i \in I}\left|\lambda_{i}\right| \chi_{U_{i}}\left|Y\|=\|\left\{\lambda_{i}\right\}_{i \in I}\right| Y^{b}\right\| .
$$

The embeddings for $Y^{\natural}$ follow with the isometry (2.3).

The weights $\omega^{b}$ and $\omega^{\natural}$ also occur in the following result.

Corollary 2.9. For every $j \in I$ the evaluation $E_{j}:\left\{\lambda_{i}\right\}_{i \in I} \mapsto \lambda_{j}$ is a bounded functional on $Y^{b}$ and $Y^{\natural}$ with $\left\|E_{j} \mid Y^{b} \rightarrow \mathbb{C}\right\| \leqslant\left(\omega^{b}(j)\right)^{-1}$ and $\left\|E_{j} \mid Y^{\natural} \rightarrow \mathbb{C}\right\| \leqslant\left(\omega^{\natural}(j)\right)^{-1}$. 
Proof. For $Y^{b}$ this follows directly from (2.4). The argument for $Y^{\natural}$ is similar.

Now we are ready to give the proof of Proposition 2.7

Proof. [Proof of Proposition 2.7] We prove the completeness of $Y^{b}$. The result for $Y^{\natural}$ follows then with the isometry (2.3). A Cauchy sequence $\left(\Lambda_{n}\right)_{n \in \mathbb{N}}$ in $Y^{b}$ is also a Cauchy sequence in $\ell_{\infty}^{\omega^{b}}$ by Lemma 2.8. Let $\Lambda$ be the limit in $\ell_{\infty}^{\omega^{b}}$. We show that $\Lambda \in Y^{b}$ and $\Lambda=\lim _{n \rightarrow \infty} \Lambda_{n}$ in the quasi-norm of $Y^{b}$. For this task let us introduce the auxiliary operator $A(\Lambda):=\sum_{i \in I}|\Lambda(i)| \chi_{U_{i}}$, which maps $\Lambda \in \mathbb{C}^{I}$ to a nonnegative measurable function on $X$.

For $\alpha \in \mathbb{C}$ and $\Lambda, \Lambda_{1}, \Lambda_{2} \in \mathbb{C}^{I}$ we have $A(\alpha \Lambda)=|\alpha| A(\Lambda)$ and $A\left(\Lambda_{1}+\Lambda_{2}\right) \leqslant A\left(\Lambda_{1}\right)+A\left(\Lambda_{2}\right)$. We also have

$$
\left|A\left(\Lambda_{1}\right)-A\left(\Lambda_{2}\right)\right| \leqslant \sum_{i \in I}|| \Lambda_{1}(i)|-| \Lambda_{2}(i)|| \chi_{U_{i}} \leqslant \sum_{i \in I}\left|\Lambda_{1}(i)-\Lambda_{2}(i)\right| \chi_{U_{i}}=A\left(\Lambda_{1}-\Lambda_{2}\right) .
$$

A sequence $\Lambda \in \mathbb{C}^{I}$ belongs to $Y^{b}$ if and only if $A(\Lambda) \in Y$, and we have the identity

$$
\left\|\Lambda\left|Y^{\mathrm{b}}\|=\| A(\Lambda)\right| Y\right\|
$$

Since $\Lambda$ is the limit of $\left(\Lambda_{n}\right)_{n \in \mathbb{N}}$ in $\ell_{\infty}^{\omega^{b}}$ it holds $\lim _{n \rightarrow \infty}\left|\Lambda(i)-\Lambda_{n}(i)\right|=0$ for all $i \in I$. Considering the local finiteness of the sum in the definition of $A$ it follows that

$$
\lim _{n \rightarrow \infty} A\left(\Lambda-\Lambda_{n}\right)(x)=0 \quad \text { for all } x \in X .
$$

The rest of the proof relies solely on Properties (2.5)-(2.7) of the operator $A$ and the solidity and completeness of $Y$. First we show $A(\Lambda) \in Y$ which is equivalent to $\Lambda \in Y^{b}$ according to (2.6). The sequence $\left(A\left(\Lambda_{n}\right)\right)_{n \in \mathbb{N}}$ is a Cauchy sequence in $Y$ because with (2.5) we can estimate $\left\|A\left(\Lambda_{n}\right)-A\left(\Lambda_{m}\right)\left|Y\|\leqslant\| A\left(\Lambda_{n}-\Lambda_{m}\right)\right| Y\right\|=\left\|\Lambda_{n}-\Lambda_{m} \mid Y^{b}\right\|$. Furthermore, from (2.7) and (2.5) it follows $\lim _{n \rightarrow \infty} A\left(\Lambda_{n}\right)(x)=A(\Lambda)(x)$ for all $x \in X$. Since $Y$ is complete we can conclude with Lemma 2.2 that $A\left(\Lambda_{n}\right) \rightarrow A(\Lambda)$ in $Y$ and $A(\Lambda) \in Y$. Finally we show $\Lambda=\lim _{n \rightarrow \infty} \Lambda_{n}$ in $Y^{b}$. The sequence $\left(A\left(\Lambda_{n}-\Lambda\right)\right)_{n \in \mathbb{N}}$ is a Cauchy sequence in $Y$, because with (2.5) we get

$$
\left\|A\left(\Lambda_{n}-\Lambda\right)-A\left(\Lambda_{m}-\Lambda\right)\left|Y\|\leqslant\| A\left(\Lambda_{n}-\Lambda_{m}\right)\right| Y\right\|=\left\|\Lambda_{n}-\Lambda_{m} \mid Y^{b}\right\| .
$$

Using (2.7) and Lemma 2.2 we deduce $A\left(\Lambda_{n}-\Lambda\right) \rightarrow 0$ in $Y$. In view of (2.6) this finishes the proof.

We finally study sequence spaces where the finite sequences are a dense subset. Since $Y^{b}$ and $Y^{\natural}$ are isometrically isomorphic via the isometry $I_{b}^{\natural}$ from (2.3), and since $I_{b}^{\natural}$ is a bijection on the sequences with finite support, these are dense in $Y^{b}$ if and only if they are dense in $Y^{\natural}$. The next result occurs in [24, Thm. 5.2] in the context of Banach spaces. However, the boundedness of the functions required there is not necessary.

Lemma 2.10. If the functions with compact support are dense in $Y$ the finite sequences are dense in $Y^{b}(\mathcal{U})$ and $Y^{\natural}(\mathcal{U})$.

Proof. Let $\Lambda=\left\{\lambda_{i}\right\}_{i \in I} \in Y^{b}$ and fix $\varepsilon>0$. Then $F:=\sum_{i \in I}\left|\lambda_{i}\right| \chi_{U_{i}} \in Y$ and there exists a function $G \in Y$ with compact support $K$ such that $\|F-G \mid Y\|<\varepsilon$. As the covering $\mathcal{U}=\left\{U_{i}\right\}_{i \in I}$ is locally finite, the index set $J:=\left\{i \in I: U_{i} \cap K \neq \varnothing\right\}$ is finite. Let $\tilde{\Lambda}$ be the sequence which coincides with $\Lambda$ on $J$ and vanishes elsewhere. Then $\tilde{F}:=\sum_{i \in J}\left|\lambda_{i}\right| \chi_{U_{i}} \in Y$ and $|F-\tilde{F}| \leqslant|F-G|$. Using the solidity of $Y$ we conclude $\left\|\Lambda-\tilde{\Lambda}\left|Y^{b}\|=\| F-\tilde{F}\right| Y\right\| \leqslant\|F-G \mid Y\|<\varepsilon$.

For a countably infinite sequence $\Lambda=\left\{\lambda_{i}\right\}_{i \in I}$, a bijection $\sigma: \mathbb{N} \rightarrow I$ and $n \in \mathbb{N}$ we define $\Lambda_{n}^{\sigma}$ as the sequence which coincides with $\Lambda$ on $\sigma(\{1, \ldots, n\})$ and is zero elsewhere. 
Lemma 2.11. Let $\mathcal{U}=\left\{U_{i}\right\}_{i \in I}$ be an admissible covering and assume that there is a bijection $\sigma: \mathbb{N} \rightarrow I$. The finite sequences are dense in $Y^{b}(\mathcal{U})$ if and only if for all $\Lambda \in Y^{b}(\mathcal{U})$ it holds $\Lambda_{n}^{\sigma} \rightarrow \Lambda$ in the quasi-norm of $Y^{b}(\mathcal{U})$ for $n \rightarrow \infty$.

Proof. Assume that the finite sequences are dense. For $n \in \mathbb{N}$ we can then choose a finite sequence $\Gamma_{n} \in Y^{b}$ with $\left\|\Gamma_{n}-\Lambda \mid Y^{b}\right\|<2^{-n}$. By solidity of $Y$ we get for $N \geqslant 1+\max \{k \in$ $\left.\mathbb{N} \mid \Gamma_{n}(\sigma(k)) \neq 0\right\}$, with the convention $\max \varnothing=0$, the estimate $\left\|\Lambda_{N}^{\sigma}-\Lambda\left|Y^{b}\|\leqslant\| \Gamma_{n}-\Lambda\right| Y^{b}\right\|<$ $2^{-n}$. The other direction is trivial.

We end this paragraph with an illustration and examine the sequence spaces associated to the weighted Lebesgue space $L_{p}^{\nu}(X)$, defined by $\left\|F\left|L_{p}^{\nu}(X)\|:=\| F \nu\right| L_{p}(X)\right\|<\infty$, where $\nu$ is a weight and $0<p \leqslant \infty$. In this special case we have a stronger statement than Lemma 2.8

Proposition 2.12. Let $\mathcal{U}=\left\{U_{i}\right\}_{i \in I}$ be an admissible covering of $X, \nu$ be a weight and $0<$ $p \leqslant \infty$. Then for $Y=L_{p}^{\nu}(X)$ we have $Y^{b}(\mathcal{U})=\ell_{p}^{\nu_{p}^{b}}(I)$ and $Y^{\natural}(\mathcal{U})=\ell_{p}^{\nu_{p}^{\natural}}(I)$ with weights given by $\nu_{p}^{b}(i):=\left\|\chi_{U_{i}} \mid L_{p}^{\nu}(X)\right\|$ and $\nu_{p}^{\natural}(i):=\mu\left(U_{i}\right)^{-1} \nu_{p}^{b}(i)$ for $i \in I$.

Proof. We give the proof for $0<p<\infty$ and $Y=L_{p}^{\nu}(X)$. For $\left\{\lambda_{i}\right\}_{i \in I} \in \mathbb{C}^{I}$ we can estimate

$$
\begin{gathered}
\left\|\left\{\lambda_{i}\right\}_{i \in I}\left|Y^{b}\left\|^{p}=\right\| \sum_{i \in I}\right| \lambda_{i}\left|\chi_{U_{i}}\right| Y\right\|^{p}=\int_{X}\left|\sum_{i \in I}\right| \lambda_{i}\left|\chi_{U_{i}}(y) \nu(y)\right|^{p} d \mu(y) \\
=\int_{X} \sum_{i \in I}\left|\lambda_{i}\right|^{p} \chi_{U_{i}}(y)^{p} \nu(y)^{p} d \mu(y)=\sum_{i \in I}\left|\lambda_{i}\right|^{p} \int_{X} \chi_{U_{i}}(y)^{p} \nu(y)^{p} d \mu(y)=\sum_{i \in I}\left|\lambda_{i}\right|^{p} \nu_{p}^{b}(i)^{p},
\end{gathered}
$$

where we used that the intersection number $\sigma(\mathcal{U})$ is finite and the equivalence of the $p$-norm and the 1-norm on $\mathbb{C}^{\sigma(\mathcal{U})}$. Applying the isometry (2.3) yields the result for $Y^{\natural}$.

\subsection{Voice transform extension}

For the definition of the coorbit spaces, we need a sufficiently large reservoir for the voice transform. Hence we extend it in this paragraph following [24]. For a weight $\nu: X \rightarrow[1, \infty)$ we introduce the space $\mathcal{H}_{1}^{\nu}:=\left\{f \in \mathcal{H}: V_{\mathcal{F}} f \in L_{1}^{\nu}(X, \mu)\right\}$. Since $\mathcal{F}$ is total in $\mathcal{H}$ by Lemma 2.1 it is easy to verify that $\left\|f\left|\mathcal{H}_{1}^{\nu}\|:=\| V_{\mathcal{F}} f\right| L_{1}^{\nu}\right\|$ constitutes a norm on $\mathcal{H}_{1}^{\nu}$. Further, we define the kernel algebra

$$
\mathcal{A}_{1}:=\left\{K: X \times X \rightarrow \mathbb{C}: K \text { is measurable and }\left\|K \mid \mathcal{A}_{1}\right\|<\infty\right\},
$$

where $\quad\left\|K \mid \mathcal{A}_{1}\right\|:=\max \left\{\underset{x \in X}{\operatorname{ess} \sup _{X}} \int_{X}|K(x, y)| d \mu(y), \underset{y \in X}{\operatorname{ess} \sup } \int_{X}|K(x, y)| d \mu(x)\right\}$.

Associated to $\nu$ is a weight $m_{\nu}$ on $X \times X$ given by

$$
m_{\nu}(x, y)=\max \left\{\frac{\nu(x)}{\nu(y)}, \frac{\nu(y)}{\nu(x)}\right\}, \quad x, y \in X .
$$

The corresponding sub-algebra $\mathcal{A}_{m_{\nu}} \subset \mathcal{A}_{1}$ is defined as

$$
\mathcal{A}_{m_{\nu}}:=\left\{K: X \times X \rightarrow \mathbb{C}: K m_{\nu} \in \mathcal{A}_{1}\right\}
$$

and endowed with the norm $\left\|K\left|\mathcal{A}_{m_{\nu}}\|:=\| K m_{\nu}\right| \mathcal{A}_{1}\right\|$. Note that a kernel $K \in \mathcal{A}_{m_{\nu}}$ operates continuously on $L_{1}^{\nu}(X)$ and $L_{\infty}^{1 / \nu}(X)$ with $\left\|K\left|L_{1}^{\nu}(X) \rightarrow L_{1}^{\nu}(X)\|\| K,\right| L_{\infty}^{1 / \nu}(X) \rightarrow L_{\infty}^{1 / \nu}(X)\right\| \leqslant$ $\left\|K \mid \mathcal{A}_{m_{\nu}}\right\|$. Technically, the theory rests upon (mapping) properties of certain kernel functions. A first example of a typical result is given by the following lemma. 
Lemma 2.13. Assume that for a family $\mathcal{G}=\left\{\psi_{x}\right\}_{x \in X} \subset \mathcal{H}$ the Gramian kernel

$$
G[\mathcal{G}, \mathcal{F}](x, y):=\left\langle\varphi_{y}, \psi_{x}\right\rangle \quad x, y \in X,
$$

is contained in $\mathcal{A}_{m_{\nu}}$. Then $\psi_{x} \in \mathcal{H}_{1}^{\nu}$ with $\left\|\psi_{x}\left|\mathcal{H}_{1}^{\nu}\|\leqslant\| G[\mathcal{G}, \mathcal{F}]\right| \mathcal{A}_{m_{\nu}}\right\| \nu(x)$ for a.e. $x \in X$.

Proof. We have $\left\|G[\mathcal{G}, \mathcal{F}]\left|\mathcal{A}_{m_{\nu}} \| \geqslant \int_{X}\right| V_{\mathcal{F}} \psi_{x}(y) \mid \frac{\nu(y)}{\nu(x)} d \mu(y)=\frac{\left\|\psi_{x} \mid \mathcal{H}_{1}^{\nu}\right\|}{\nu(x)}\right.$ for a.e. $x \in X$.

The theory in [24, 50] is developed under the global assumption that $\mathcal{F}$ is uniformly bounded, i.e. $\left\|\varphi_{x}\right\| \leqslant C_{B}$ for all $x \in X$ and some $C_{B}>0$. This assumption can be weakened.

Lemma 2.14. Let $\nu \geqslant 1$ be a weight such that the analyzing frame $\mathcal{F}$ satisfies

(i) $\left\|\varphi_{x} \mid \mathcal{H}\right\| \leqslant C_{B} \nu(x)$ for some constant $C_{B}>0$ and all $x \in X$,

(ii) $R_{\mathcal{F}} \in \mathcal{A}_{m_{\nu}}$.

Then $\mathcal{H}_{1}^{\nu}$ is a Banach space and the canonical embedding $\mathcal{H}_{1}^{\nu} \hookrightarrow \mathcal{H}$ is continuous and dense. Moreover, there is a subset $X^{*} \subset X$ such that $\varphi_{x} \in \mathcal{H}_{1}^{\nu}$ for every $x \in X^{*}$ and $\mu\left(X \backslash X^{*}\right)=0$. The corresponding map $\Psi: X^{*} \rightarrow \mathcal{H}_{1}^{\nu}, x \mapsto \varphi_{x}$ is Bochner-measurable in $\mathcal{H}_{1}^{\nu}$.

Proof. A Cauchy sequence $\left(f_{n}\right)_{n \in \mathbb{N}} \subset \mathcal{H}_{1}^{\nu}$ determines a Cauchy sequence $\left(F_{n}:=V f_{n}\right)_{n \in \mathbb{N}}$ in $L_{1}^{\nu}$, which converges to some $F \in L_{1}^{\nu}$. Since the kernel $R \in \mathcal{A}_{m_{\nu}}$ operates continuously on $L_{1}^{\nu}$, the equality $F_{n}=R\left(F_{n}\right)$ for $n \in \mathbb{N}$ implies $F=R(F)$. Furthermore, because of $\left\|\varphi_{x} \mid \mathcal{H}\right\| \leqslant C_{B} \nu(x)$ it holds $|R(x, y)| \leqslant C_{B}^{2} \nu(x) \nu(y)$ for all $x, y \in X$ and we can deduce

$$
|F(x)|=\left|\int_{X} R(x, y) F(y) d \mu(y)\right| \leqslant C_{B}^{2} \nu(x) \int_{X}|F(y)| \nu(y) d \mu(y)=C_{B}^{2} \nu(x)\left\|F \mid L_{1}^{\nu}\right\| .
$$

This shows $F \in L_{\infty}^{1 / \nu}$, and as $L_{\infty}^{1 / \nu} \cap L_{1}^{\nu} \subset L_{2}$ even $F \in L_{2}$. The reproducing formula on $\mathcal{H}$ yields $f \in \mathcal{H}$ with $V f=F \in L_{1}^{\nu}$, which implies $f \in \mathcal{H}_{1}^{\nu}$. Since $\left\|f_{n}-f\left|\mathcal{H}_{1}^{\nu}\|=\| F_{n}-F\right| L_{1}^{\nu}\right\|$ we obtain $f_{n} \rightarrow f$ in $\mathcal{H}_{1}^{\nu}$. This proves the completeness. To prove the continuity of the embedding we observe $\left\|h\left|\mathcal{H}\left\|^{2}=\right\| V h\right| L_{2}\right\|^{2} \leqslant\left\|V h\left|L_{\infty}^{1 / \nu}\|\| h\right| \mathcal{H}_{1}^{\nu}\right\|$ for $h \in \mathcal{H}_{1}^{\nu}$. Together with $\left\|V h \mid L_{\infty}^{1 / \nu}\right\| \leqslant$ $\sup _{x \in X}\left\{\frac{\left\|\varphi_{x} \mid \mathcal{H}\right\|}{\nu(x)}\|h \mid \mathcal{H}\|\right\} \leqslant C_{B}\|h \mid \mathcal{H}\|$, where $\left\|\varphi_{x} \mid \mathcal{H}\right\| \leqslant C_{B} \nu(x)$ was used, the continuity follows. Due to Lemma 2.13, applied with $\mathcal{G}=\mathcal{F}$, there is a null-set $N \subset X$ such that $\varphi_{x} \in \mathcal{H}_{1}^{\nu}$ for every $x \in X^{*}:=X \backslash N$. The density of $\mathcal{H}_{1}^{\nu} \hookrightarrow \mathcal{H}$ is thus a consequence of the totality of $\left\{\varphi_{x}\right\}_{x \in X^{*}}$ in $\mathcal{H}$, as stated by Lemma 2.1. It remains to prove the Bochner-measurability of $\Psi$. Since $V_{\mathcal{F}}: \mathcal{H}_{1}^{\nu} \rightarrow V_{\mathcal{F}}\left(\mathcal{H}_{1}^{\nu}\right)$ is an isometric isomorphism, it suffices to confirm that

$$
\widetilde{\Psi}:=V_{\mathcal{F}} \circ \Psi: X^{*} \rightarrow L_{1}^{\nu}(X), x \mapsto V_{\mathcal{F}} \varphi_{x}
$$

is Bochner-measurable in $L_{1}^{\nu}(X)$. The proof of this is divided into three steps.

Step 1: Let us first construct an adequate partition of $X$. Since $\mu$ is a Radon measure, by definition locally finite, all compact subsets of $X$ have finite measure. As $X$ is assumed to be $\sigma$-compact, the measure $\mu$ is thus $\sigma$-finite. Hence $X=\bigcup_{n \in \mathbb{N}} L_{n}$ for certain subsets $L_{n} \subset X$ of finite measure. By subdividing each of these sets further into $L_{n, m}:=\left\{x \in L_{n}: \nu(x) \leqslant m\right\}$, disjointifying these subdivided sets, and finally by renumbering the resulting countable family of sets, we obtain a sequence $\left(K_{n}\right)_{n \in \mathbb{N}}$ of pairwise disjoint sets of finite measure with $X=\bigcup_{n \in \mathbb{N}} K_{n}$ and such that $\nu(x) \leqslant C_{n}$ holds for all $x \in K_{n}$ and suitable constants $C_{n}>0$.

Step 2: We now show that for every $n \in \mathbb{N}$ the function

$$
\widetilde{\Psi}_{n}: X^{*} \rightarrow L_{1}^{\nu}(X), x \mapsto V_{\mathcal{F}} \varphi_{x} \cdot \chi_{K_{n}}
$$


is Bochner-measurable in $L_{1}^{\nu}(X)$. To this end, let $\left(f_{\ell}\right)_{\ell \in \mathbb{N}}$ be an orthonormal basis of $\mathcal{H}$ with $f_{\ell} \in \mathcal{H}_{1}^{\nu}$ for all $\ell \in \mathbb{N}$. Such a basis exists since $\mathcal{H}$ is separable and $\mathcal{H}_{1}^{\nu}$ is a dense subspace of $\mathcal{H}$. Then we define the functions

$$
\Phi_{\ell}:=\overline{V_{\mathcal{F}} f_{\ell}} \in L_{1}^{\nu}(X) \quad \text { and } \quad G_{n, \ell}:=V_{\mathcal{F}} f_{\ell} \cdot \chi_{K_{n}} \in L_{1}^{\nu}(X) .
$$

Note that $\Phi_{\ell}(x)=\left\langle\varphi_{x}, f_{\ell}\right\rangle$ is the $\ell$-th expansion coefficient of $\varphi_{x}$ with respect to $\left(f_{\ell}\right)_{\ell \in \mathbb{N}}$. Due to the measurability of $\Phi_{\ell} \in L_{1}^{\nu}(X)$ the function $x \mapsto \Phi_{\ell}(x) G_{n, \ell}$ is clearly Bochner-measurable. Since the pointwise limit of Bochner-measurable functions is again Bochner-measurable, Step 2 is finished if we can show that for every fixed $x \in X^{*}$

$$
\widetilde{\Psi}_{n}(x)=\lim _{N \rightarrow \infty} \sum_{\ell=1}^{N} \Phi_{\ell}(x) G_{n, \ell} \quad \text { in } L_{1}^{\nu}(X) .
$$

This follows with Lebesgue's dominated convergence theorem: For every $y \in X$ we have

$$
\lim _{N \rightarrow \infty} \sum_{\ell=1}^{N} \Phi_{\ell}(x) G_{n, \ell}(y)=\lim _{N \rightarrow \infty} V_{\mathcal{F}}\left(\sum_{\ell=1}^{N} \Phi_{\ell}(x) f_{\ell}\right)(y) \cdot \chi_{K_{n}}(y)=V_{\mathcal{F}} \varphi_{x}(y) \cdot \chi_{K_{n}}(y)=\widetilde{\Psi}_{n}(y) .
$$

Note here that $\varphi_{x}=\sum_{\ell=1}^{\infty} \Phi_{\ell}(x) f_{\ell}$ with convergence in $\mathcal{H}$, and in general $V_{\mathcal{F}} g_{N}(x) \rightarrow V_{\mathcal{F}} g(x)$ for fixed $x \in X$ if $g_{N} \rightarrow g$ in $\mathcal{H}$. Finally, we estimate using $\left\|\varphi_{x} \mid \mathcal{H}\right\| \leqslant C_{B} \nu(x)$

$$
\begin{aligned}
\left|\sum_{\ell=1}^{N} \Phi_{\ell}(x) G_{n, \ell}(y)\right| & \leqslant\left(\sum_{\ell=1}^{N}\left|\Phi_{\ell}(x)\right|^{2}\right)^{\frac{1}{2}}\left(\sum_{\ell=1}^{N}\left|G_{n, \ell}(y)\right|^{2}\right)^{\frac{1}{2}} \\
& \leqslant\left\|\varphi_{x}\left|\mathcal{H}\|\| \varphi_{y}\right| \mathcal{H}\right\| \chi_{K_{n}}(y) \leqslant C_{B} \nu(y)\left\|\varphi_{x}\left|\mathcal{H}\left\|\chi_{K_{n}}(y) \leqslant C_{B} C_{n}\right\| \varphi_{x}\right| \mathcal{H}\right\| \chi_{K_{n}}(y) .
\end{aligned}
$$

Since $K_{n}$ is of finite measure this provides an integrable majorant (with respect to $y$ ).

Step 3: Similar to Step 2 the Bochner-measurability of $\widetilde{\Psi}$ is proved by showing for $x \in X^{*}$

$$
\widetilde{\Psi}(x)=\lim _{N \rightarrow \infty} \sum_{n=1}^{N} \widetilde{\Psi}_{n}(x) \text { in } L_{1}^{\nu}(X) .
$$

The pointwise limit is obvious: For every $y \in X$ we clearly have

$$
[\tilde{\Psi}(x)](y)=V_{\mathcal{F}} \varphi_{x}(y)=\lim _{N \rightarrow \infty} \sum_{n=1}^{N} \chi_{K_{n}}(y) \cdot V_{\mathcal{F}} \varphi_{x}(y)=\lim _{N \rightarrow \infty} \sum_{n=1}^{N}\left[\tilde{\Psi}_{n}(x)\right](y) .
$$

Using Lebesgue's dominated convergence theorem with majorant $|\widetilde{\Psi}(x)|$ proves the claim.

Under the assumptions (2.10) we therefore have the chain of continuous embeddings

$$
\left.\mathcal{H}_{1}^{\nu} \stackrel{i}{\hookrightarrow} \mathcal{H} \stackrel{i *}{\hookrightarrow}\left(\mathcal{H}_{1}^{\nu}\right)\right\urcorner
$$

where $\left.\left(\mathcal{H}_{1}^{\nu}\right)\right\urcorner$ denotes the normed anti-dual of $\mathcal{H}_{1}^{\nu}$, which plays the role of the tempered distributions in this abstract context. Moreover, there is a subset $X^{*} \subset X$ with $\mu\left(X \backslash X^{*}\right)=0$ such that $\varphi_{x} \in \mathcal{H}_{1}^{\nu}$ for $x \in X^{*}$. Hence we may extend the transform $V_{\mathcal{F}}: \mathcal{H} \rightarrow L_{2}(X)$ to $\left(\mathcal{H}_{1}^{\nu}\right)^{\urcorner}$by

$$
\left.V_{\mathcal{F}} f(x)=\left\langle f, \varphi_{x}\right\rangle, \quad x \in X^{*}, f \in\left(\mathcal{H}_{1}^{\nu}\right)\right\urcorner,
$$

where $\langle\cdot, \cdot\rangle$ denotes the duality product on $\left(\mathcal{H}_{1}^{\nu}\right)^{\urcorner} \times \mathcal{H}_{1}^{\nu}$. The anti-dual is used so that this product extends the scalar product of $\mathcal{H}$. 
Lemma 2.15. Under the assumptions (2.10) the extension (2.11) is a well-defined continuous mapping $\left.V_{\mathcal{F}}:\left(\mathcal{H}_{1}^{\nu}\right)\right\urcorner \rightarrow L_{\infty}^{1 / \nu}(X)$.

Proof. Let $\left.f \in\left(\mathcal{H}_{1}^{\nu}\right)\right\urcorner$. The function $V_{\mathcal{F}} f(x)=\left\langle f, \varphi_{x}\right\rangle$ is well-defined for every $x \in X^{*}$. It determines a measurable function on $X$, in the sense of equivalence classes, due to the Bochner measurability of $x \mapsto \varphi_{x}$ in $\mathcal{H}_{1}^{\nu}$ proved in Lemma 2.14. Using Lemma 2.13 we can estimate

$$
\left|V_{\mathcal{F}} f(x)\right|=\left|\left\langle f, \varphi_{x}\right\rangle\right| \leqslant\left\|f\left|\left(\mathcal{H}_{1}^{\nu}\right)^{\urcorner}\|\| \varphi_{x}\right| \mathcal{H}_{1}^{\nu}\right\| \leqslant\left\|f\left|\left(\mathcal{H}_{1}^{\nu}\right)^{\urcorner}\|\| \mathcal{R}_{\mathcal{F}}\right| \mathcal{A}_{m_{\nu}}\right\| \nu(x) .
$$

This shows $V_{\mathcal{F}} f \in L_{\infty}^{1 / \nu}(X)$ with $\left\|V_{\mathcal{F}} f\left|L_{\infty}^{1 / \nu}\|\leqslant\| f\right|\left(\mathcal{H}_{1}^{\nu}\right)^{\urcorner}\right\|\left\|R_{\mathcal{F}} \mid \mathcal{A}_{m_{\nu}}\right\|$.

Remark 2.16. The membership $R_{\mathcal{F}} \in \mathcal{A}_{m_{\nu}}$ does not ensure $\mathcal{F} \subset \mathcal{H}_{1}^{\nu}$, wherefore the extended voice transform (2.11) might not be defined at every point $x \in X$. This detail has not been accounted for in preceding papers, and fortunately it is negligible since functions on $X$ are only determined up to $\mu$-equivalence classes. Therefore we - as in [24, 50] - will henceforth assume $\mathcal{F} \subset \mathcal{H}_{1}^{\nu}$ to simplify the exposition.

We proceed to establish the injectivity of the extended voice transform. To this end, the following characterization of the duality bracket $\langle\cdot, \cdot\rangle_{\left.\left(\mathcal{H}_{1}^{\nu}\right)\right\urcorner \times \mathcal{H}_{1}^{\nu}}$ will be useful.

Lemma 2.17. If $\mathcal{F}$ has properties (2.10), then for all $f \in\left(\mathcal{H}_{1}^{\nu}\right)$ and $g \in \mathcal{H}_{1}^{\nu}$ it holds

$$
\langle f, g\rangle_{\left.\left(\mathcal{H}_{1}^{\nu}\right)\right\urcorner \times \mathcal{H}_{1}^{\nu}}=\int_{X} V_{\mathcal{F}} f(y) \overline{V_{\mathcal{F}} g(y)} d \mu(y)=:\left\langle V_{\mathcal{F}} f, V_{\mathcal{F}} g\right\rangle_{L_{\infty}^{1 / \nu} \times L_{1}^{\nu}} .
$$

Proof. Let $f \in\left(\mathcal{H}_{1}^{\nu}\right)^{\urcorner}$and $g \in \mathcal{H}_{1}^{\nu}$. Then $V_{\mathcal{F}} g \in L_{2} \cap L_{1}^{\nu}$ and we get

$$
\begin{aligned}
\langle f, g\rangle & =\left\langle f, V_{\mathcal{F}}^{*} V_{\mathcal{F}} g\right\rangle=\left\langle f, \int_{X} V_{\mathcal{F}} g(y) \varphi_{y} d \mu(y)\right\rangle \\
& =\int_{X} \overline{V_{\mathcal{F}} g(y)}\left\langle f, \varphi_{y}\right\rangle d \mu(y)=\left\langle V_{\mathcal{F}} f, V_{\mathcal{F}} g\right\rangle_{L_{\infty}^{1 / \nu} \times L_{1}^{\nu}} .
\end{aligned}
$$

For this equality, it is important that the duality product commutes with the integral. To verify this, note that since $G:=V_{\mathcal{F}} g \in L_{1}^{\nu}$ the integral $\int_{X} G(y) \varphi_{y} d \mu(y)$ also exists in the Bochner sense in $\mathcal{H}_{1}^{\nu}$. Indeed, in view of Lemma 2.14 the integrand is Bochner-measurable in $\mathcal{H}_{1}^{\nu}$. Bochner-integrability follows then from the estimate

$$
\int_{X}|G(y)| \cdot\left\|\varphi_{y}\left|\mathcal{H}_{1}^{\nu}\|d \mu(y) \leqslant\| R_{\mathcal{F}}\right| \mathcal{A}_{m_{\nu}}\right\| \int_{X}|G(y)| \nu(y) d \mu(y)=\left\|R_{\mathcal{F}}\left|\mathcal{A}_{m_{\nu}}\|\| G\right| L_{1}^{\nu}\right\|,
$$

where Lemma 2.13 was used. Moreover, the value of the Bochner integral $h:=\int_{X} G(y) \varphi_{y} d \mu(y)$ equals $g$ since for every $\zeta \in \mathcal{H}$

$$
\langle g, \zeta\rangle=\int_{X} V_{\mathcal{F}} g(y) \cdot \overline{V_{\mathcal{F}} \zeta(y)} d \mu(y)=\langle h, \zeta\rangle .
$$

Using Lemma 2.17 we can simplify the proof of [24, Lem. 3.2].

Lemma 2.18. Assume that the analyzing frame $\mathcal{F}$ has properties (2.10). Then the expression $\left\|V_{\mathcal{F}} f \mid L_{\infty}^{1 / \nu}\right\|$ is an equivalent norm on $\left.\left(\mathcal{H}_{1}^{\nu}\right)\right\urcorner$. 
Proof. We already know from Lemma 2.15 that $\left\|V_{\mathcal{F}} f\left|L_{\infty}^{1 / \nu}\|\lesssim\| f\right|\left(\mathcal{H}_{1}^{\nu}\right)^{\urcorner}\right\|$. For the estimate from below we argue with the help of Lemma 2.17

$$
\begin{aligned}
\left\|f \mid\left(\mathcal{H}_{1}^{\nu}\right)^{\urcorner}\right\| & =\sup _{\left\|h \mid \mathcal{H}_{1}^{\nu}\right\|=1}\left|\langle f, h\rangle_{\left.\left(\mathcal{H}_{1}^{\nu}\right)\right\urcorner \times \mathcal{H}_{1}^{\nu}}\right|=\sup _{\left\|h \mid \mathcal{H}_{1}^{\nu}\right\|=1}\left|\left\langle V_{\mathcal{F}} f, V_{\mathcal{F}} h\right\rangle_{L_{\infty}^{1 / \nu} \times L_{1}^{\nu}}\right| \\
& \leqslant \sup _{H \in L_{1}^{\nu},\left\|H \mid L_{1}^{\nu}\right\| \leqslant 1}\left|\left\langle V_{\mathcal{F}} f, H\right\rangle_{L_{\infty}^{1 / \nu} \times L_{1}^{\nu}}\right|=\left\|V_{\mathcal{F}} f \mid L_{\infty}^{1 / \nu}\right\| .
\end{aligned}
$$

A direct consequence of this lemma is the injectivity of $V_{\mathcal{F}}$.

Corollary 2.19. The voice transform $V_{\mathcal{F}}:\left(\mathcal{H}_{1}^{\nu}\right)^{\urcorner} \rightarrow L_{\infty}^{1 / \nu}(X)$ is continuous and injective.

The injectivity of $V_{\mathcal{F}}$ on $\left(\mathcal{H}_{1}^{\nu}\right)^{\urcorner}$implies that $\mathcal{F}$ is total in $\mathcal{H}_{1}^{\nu}$.

Corollary 2.20. Let $N \subset X$ be a set of measure zero. Then $\left\{\varphi_{x}\right\}_{x \in X \backslash N}$ is total in $\mathcal{H}_{1}^{\nu}$.

Proof. If this is not the case, the closure $\mathcal{C}$ of $\operatorname{span}\left\{\varphi_{x}: x \in X \backslash N\right\}$ in $\mathcal{H}_{1}^{\nu}$ is a true subspace, and the Hahn-Banach extension theorem yields $\left.f \in\left(\mathcal{H}_{1}^{\nu}\right)\right\urcorner, f \neq 0$, with $\langle f, \zeta\rangle=0$ for all $\zeta \in \mathcal{C}$. Hence, $V_{\mathcal{F}} f(x)=0$ for a.e. $x \in X$ and therefore $f=0$ by injectivity of $V_{\mathcal{F}}$, which is true even with respect to $\mu$-equivalence classes in the image space. This is a contradiction.

The adjoint $\left.V_{\mathcal{F}}^{*}: L_{\infty}^{1 / \nu}(X) \rightarrow\left(\mathcal{H}_{1}^{\nu}\right)\right\urcorner$ of the restriction $V_{\mathcal{F}}: \mathcal{H}_{1}^{\nu} \rightarrow L_{1}^{\nu}(X)$ naturally extends the adjoint of $V_{\mathcal{F}}: \mathcal{H} \rightarrow L_{2}(X)$ due to the equality $\left\langle F, V_{\mathcal{F}} \zeta\right\rangle_{L_{\infty}^{1 / \nu} \times L_{1}^{\nu}}=\left\langle F, V_{\mathcal{F}} \zeta\right\rangle_{L_{2} \times L_{2}}$ in case $\zeta \in \mathcal{H}_{1}^{\nu}$ and $F \in L_{\infty}^{1 / \nu} \cap L_{2}$, and it can also be represented by a weak integral of the form (2.1). The relations

$$
V_{\mathcal{F}}^{*} V_{\mathcal{F}} f=f \quad \text { and } \quad V_{\mathcal{F}} V_{\mathcal{F}}^{*}(F)=R(F)
$$

remain valid for the extension, i.e., they hold for $\left.f \in\left(\mathcal{H}_{1}^{\nu}\right)\right\urcorner$ and $F \in L_{\infty}^{1 / \nu}$. Indeed, Lemma 2.17 yields $\left\langle V_{\mathcal{F}}^{*} V_{\mathcal{F}} f, \zeta\right\rangle=\left\langle V_{\mathcal{F}} f, V_{\mathcal{F}} \zeta\right\rangle_{L_{\infty}^{1 / \nu} \times L_{1}^{\nu}}=\langle f, \zeta\rangle$ for all $\zeta \in \mathcal{H}_{1}^{\nu}$. Further, we have $V_{\mathcal{F}} V_{\mathcal{F}}^{*} F(x)=$ $\left\langle V_{\mathcal{F}}^{*} F, \varphi_{x}\right\rangle=\int_{X} F(y)\left\langle\varphi_{y}, \varphi_{x}\right\rangle d \mu(y)=R(F)(x)$ for all $x \in X$.

An easy consequence of the relations (2.12) is the important fact that the reproducing formula extends to $\left(\mathcal{H}_{1}^{\nu}\right)^{\urcorner}$, a result obtained differently in [24, Lemma 3.6].

Lemma 2.21. Let $\nu \geqslant 1$ be a weight on $X$ and assume that the analyzing frame $\mathcal{F}$ satisfies (2.10). Then $V_{\mathcal{F}} f(x)=R\left(V_{\mathcal{F}} f\right)(x)$ for every $\left.f \in\left(\mathcal{H}_{1}^{\nu}\right)\right\urcorner$ and $x \in X$. Conversely, if $F \in L_{\infty}^{1 / \nu}(X)$ satisfies $F=R(F)$ then there is a unique $\left.f \in\left(\mathcal{H}_{1}^{\nu}\right)\right\urcorner$ such that $F=V_{\mathcal{F}} f$.

Proof. According to (2.12) we have $R(V f)=V V^{*} V f=V f$ for $\left.f \in\left(\mathcal{H}_{1}^{\nu}\right)\right\urcorner$. For the opposite direction assume that $F \in L_{\infty}^{1 / \nu}$ satisfies $F=R(F)$. Then by (2.12) the element $\left.V^{*} F \in\left(\mathcal{H}_{1}^{\nu}\right)\right\urcorner$ has the property $V V^{*} F=R(F)=F$. It is unique since $V$ is injective on $\left(\mathcal{H}_{1}^{\nu}\right)^{\urcorner}$.

Finally we state the correspondence between the weak*-convergence of a net $\left(f_{i}\right)_{i \in I}$ in $\left(\mathcal{H}_{1}^{\nu}\right)$ and the pointwise convergence of $\left(V_{\mathcal{F}} f_{i}\right)_{i \in I}$ (compare [24, Lem. 3.6]).

Lemma 2.22. Let $\left(f_{i}\right)_{i \in I}$ be a net in $\left(\mathcal{H}_{1}^{\nu}\right)^{\urcorner}$.

(i) If $\left(f_{i}\right)_{i \in I}$ converges to some $f \in\left(\mathcal{H}_{1}^{\nu}\right)^{\urcorner}$in the weak ${ }^{*}$-topology of $\left(\mathcal{H}_{1}^{\nu}\right)^{\urcorner}$, then $\left(V_{\mathcal{F}} f_{i}\right)_{i \in I}$ converges pointwise to $V_{\mathcal{F}} f$ everywhere. 
(ii) If $\left(V_{\mathcal{F}} f_{i}\right)_{i \in I}$ converges pointwise a.e. to a function $F: X \rightarrow \mathbb{C}$ and if $\left(f_{i}\right)_{i \in I}$ is uniformly bounded in $\left(\mathcal{H}_{1}^{\nu}\right)^{\urcorner}$, then $\left(f_{i}\right)_{i \in I}$ converges to some $f \in\left(\mathcal{H}_{1}^{\nu}\right)^{\urcorner}$in the weak ${ }^{*}$-topology with $V_{\mathcal{F}} f=F$ a.e.

Proof. We give a proof for sequences $\left(f_{n}\right)_{n \in \mathbb{N}}$ which extends straightforwardly to nets. Part (i): The weak*-convergence implies $\left\langle f_{n}, \varphi_{x}\right\rangle \rightarrow\left\langle f, \varphi_{x}\right\rangle$ for $n \rightarrow \infty$ and all $x \in X$.

Part (ii): Let $X^{*} \subset X$ denote the subset where the sequence $\left(V_{\mathcal{F}} f_{n}\right)_{n \in \mathbb{N}}$ converges pointwise. The space $M=\operatorname{span}\left\{\varphi_{x}: x \in X^{*}\right\}$ lies dense in $\mathcal{H}_{1}^{\nu}$ by Corollary 2.20. We define a conjugatelinear functional $\tilde{f}$ on $M$ by $\tilde{f}(h):=\lim _{n \rightarrow \infty}\left\langle f_{n}, h\right\rangle$ for $h \in M$. By assumption, there is $C>0$ so that $\left\|f_{n} \mid\left(\mathcal{H}_{1}^{\nu}\right)^{\urcorner}\right\| \leqslant C$, which leads to $\left|\left\langle f_{n}, h\right\rangle\right| \leqslant\left\|f_{n}\left|\left(\mathcal{H}_{1}^{\nu}\right)^{\urcorner}\|\| h\right| \mathcal{H}_{1}^{\nu}\right\| \leqslant C\left\|h \mid \mathcal{H}_{1}^{\nu}\right\|$ for all $n \in \mathbb{N}$ and shows that $\tilde{f}$ is bounded on $M$ with respect to $\left\|\cdot \mid \mathcal{H}_{1}^{\nu}\right\|$. Hence it can be uniquely extended to some $f \in\left(\mathcal{H}_{1}^{\nu}\right)^{\urcorner}$. For $\varepsilon>0$ and $\zeta \in \mathcal{H}_{1}^{\nu}$ we choose $h \in M$ such that $\left\|h-\zeta \mid \mathcal{H}_{1}^{\nu}\right\|<\varepsilon$. We get

$$
\left|\left\langle f_{n}-f, \zeta\right\rangle\right| \leqslant\left\|\zeta-h\left|\mathcal{H}_{1}^{\nu}\|\cdot\| f_{n}-f\right|\left(\mathcal{H}_{1}^{\nu}\right)^{\urcorner}\right\|+\left|\left\langle f_{n}-f, h\right\rangle\right| \leqslant \varepsilon\left(C+\left\|f \mid\left(\mathcal{H}_{1}^{\nu}\right)^{\urcorner}\right\|\right)+\left|\left\langle f_{n}-f, h\right\rangle\right| .
$$

Letting $n \rightarrow \infty$ it follows $\lim \sup _{n \rightarrow \infty}\left|\left\langle f_{n}-f, \zeta\right\rangle\right| \leqslant \varepsilon\left(C+\left\|f \mid\left(\mathcal{H}_{1}^{\nu}\right)^{\urcorner}\right\|\right)$. This holds for all $\varepsilon>0$, hence, $\lim _{n \rightarrow \infty}\left|\left\langle f_{n}-f, \zeta\right\rangle\right|=0$. This shows that $f_{n} \rightarrow f$ in the weak*-topology of $\left(\mathcal{H}_{1}^{\nu}\right)^{\urcorner}$. As a consequence $V_{\mathcal{F}} f(x)=\left\langle f, \varphi_{x}\right\rangle=\lim _{n \rightarrow \infty}\left\langle f_{n}, \varphi_{x}\right\rangle=\lim _{n \rightarrow \infty} V_{\mathcal{F}} f_{n}(x)=F(x)$ for all $x \in X^{*}$.

A direct implication is the correspondence principle with respect to sums formulated below.

Corollary 2.23. If $\sum_{i \in I} f_{i}$ converges unconditionally in the weak*-topology of $\left(\mathcal{H}_{1}^{\nu}\right)^{\urcorner}$then the series $\sum_{i \in I} V_{\mathcal{F}} f_{i}(x)$ converges absolutely for all $x \in X$. Conversely, if $\sum_{i \in I} V_{\mathcal{F}} f_{i}(x)$ converges absolutely for a.e. $x \in X$ and if the finite partial sums of $\sum_{i \in I} f_{i}$ are uniformly bounded in $\left(\mathcal{H}_{1}^{\nu}\right)^{\urcorner}$ then $\sum_{i \in I} f_{i}$ converges unconditionally in the weak*-topology.

\subsection{Coorbit spaces}

In this central part we introduce the notion of coorbit spaces, building upon the correspondence between elements of $\left.\left(\mathcal{H}_{1}^{\nu}\right)\right\urcorner$ and functions on $X$ as established by the transform $V_{\mathcal{F}}$. The idea is to characterize $\left.f \in\left(\mathcal{H}_{1}^{\nu}\right)\right\urcorner$ by properties of the corresponding function $V_{\mathcal{F}} f$. For a viable theory the analyzing frame $\mathcal{F}$ must fulfill certain suitability conditions with respect to $Y$.

Definition 2.24. Let $\nu \geqslant 1$ be a weight on $X$. We say that $\mathcal{F}$ has property $F(\nu, Y)$ if it satisfies condition (2.10) and if the following holds true,

(i) $R_{\mathcal{F}}: Y \rightarrow Y$ acts continuously on $Y$,

(ii) $R_{\mathcal{F}}(Y) \hookrightarrow L_{\infty}^{1 / \nu}(X)$.

Condition (2.10) ensures that the voice transform extends to $\left.\left(\mathcal{H}_{1}^{\nu}\right)\right\urcorner$. Further, conditions (i) and (ii) imply that $R_{\mathcal{F}} F(x)=\int_{X} R_{\mathcal{F}}(x, y) F(y) d \mu(y)$ is well-defined for a.e. $x \in X$ if $F \in Y$. In addition, also due to (i) and (ii), the operator $R_{\mathcal{F}}: Y \rightarrow L_{\infty}^{1 / \nu}(X)$ is continuous: For $F \in Y$ we have $R(F) \in L_{\infty}^{1 / \nu}(X)$ and

$$
\left\|R(F)\left|L_{\infty}^{1 / \nu}\|\lesssim\| R(F)\right| Y\right\| \leqslant\|R|Y \rightarrow Y\|\cdot\| F| Y\| .
$$

In view of Definition 2.24 it makes sense to introduce the following subalgebra of $\mathcal{A}_{m_{\nu}}$ from (2.8)

$$
\mathcal{B}_{Y, m_{\nu}}=\left\{K: X \times X \rightarrow \mathbb{C}: K \in \mathcal{A}_{m_{\nu}} \text { and } K \text { is bounded from } Y \rightarrow Y\right\},
$$


equipped with the quasi-norm $\left\|K \mid \mathcal{B}_{Y, m_{\nu}}\right\|:=\max \left\{\left\|K\left|\mathcal{A}_{m_{\nu}}\|\| K,\right| Y \rightarrow Y\right\|\right\}$.

Now we are able to give the definition of the coorbit of a rich solid QBF-space $Y$.

Definition 2.25. Let $Y$ be a rich solid QBF-space on $X$ and assume that the analyzing frame $\mathcal{F}=\left\{\varphi_{x}\right\}_{x \in X}$ has property $F(\nu, Y)$ for some weight $\nu: X \rightarrow[1, \infty)$. The coorbit of $Y$ with respect to $\mathcal{F}$ is defined by

$$
\left.\operatorname{Co}(\nu, \mathcal{F}, Y):=\left\{f \in\left(\mathcal{H}_{1}^{\nu}\right)\right\urcorner: V_{\mathcal{F}} f \in Y\right\} \quad \text { with quasi-norm } \quad\left\|f\left|\operatorname{Co}(\nu, \mathcal{F}, Y)\|:=\| V_{\mathcal{F}} f\right| Y\right\| .
$$

Since the coorbit is independent of the weight $\nu$ in the definition, as proved by the lemma below, it is omitted in the notation and we simply write $\operatorname{Co}(\mathcal{F}, Y):=\operatorname{Co}(\nu, \mathcal{F}, Y)$. Moreover, if the analyzing frame $\mathcal{F}$ is fixed we may just write $\operatorname{Co}(Y)$.

Lemma 2.26. The coorbit $\mathrm{Co}(\nu, \mathcal{F}, Y)$ does not depend on the particular weight $\nu$ chosen in the definition in the following sense. If $\tilde{\nu} \geqslant 1$ is another weight such that $\mathcal{F}$ has property $F(\tilde{\nu}, Y)$ then we have $\operatorname{Co}(\tilde{\nu}, \mathcal{F}, Y)=\operatorname{Co}(\nu, \mathcal{F}, Y)$.

Proof. If $\mathcal{F}$ has properties $F(\nu, Y)$ and $F(\tilde{\nu}, Y)$ it also has property $F(\omega, Y)$ for $\omega=\nu+\tilde{\nu}$. We show $\operatorname{Co}(\omega, Y)=\operatorname{Co}(\nu, Y)$. Since $\omega \geqslant \nu$ we have the continuous dense embedding $\mathcal{H}_{1}^{\omega} \hookrightarrow \mathcal{H}_{1}^{\nu}$ which implies $\left.\left.\left(\mathcal{H}_{1}^{\nu}\right)\right\urcorner \hookrightarrow\left(\mathcal{H}_{1}^{\omega}\right)\right\urcorner$ and hence $\operatorname{Co}(\nu, Y) \subset \operatorname{Co}(\omega, Y)$. For the opposite inclusion let $f \in \operatorname{Co}(\omega, Y)$. Then $f \in\left(\mathcal{H}_{1}^{\omega}\right)^{\urcorner}$and $F:=V f \in Y$ with $R(F) \in L_{\infty}^{1 / \nu}$ by property $F(\nu, Y)$. Since $F=R(F)$ according to the reproducing formula on $\left(\mathcal{H}_{1}^{\omega}\right)^{\urcorner}$we thus have $F \in L_{\infty}^{1 / \nu}$. The inverse reproducing formula on $\left(\mathcal{H}_{1}^{\nu}\right)^{\urcorner}$then yields $\left.\tilde{f} \in\left(\mathcal{H}_{1}^{\nu}\right)^{\urcorner} \subset\left(\mathcal{H}_{1}^{\omega}\right)\right\urcorner$ with $V \tilde{f}=F$, which due to the injectivity of $V$ is equal to $f$. This shows $\left.f \in\left(\mathcal{H}_{1}^{\nu}\right)\right\urcorner$, and as $V f \in Y$ even $f \in \operatorname{Co}(\nu, Y)$. Finally note that the quasi-norms on $\operatorname{Co}(\omega, Y)$ and $\operatorname{Co}(\nu, Y)$ are equal. Analogously it follows $\operatorname{Co}(\omega, Y)=\operatorname{Co}(\tilde{\nu}, Y)$.

Remark 2.27. The claim of Lemma[2.26 has to be understood in the sense

$$
\left\{\left.f\right|_{\left\langle\varphi_{x}: x \in X\right\rangle}: f \in \operatorname{Co}(\tilde{\nu}, \mathcal{F}, Y)\right\}=\left\{\left.f\right|_{\left\langle\varphi_{x}: x \in X\right\rangle}: f \in \operatorname{Co}(\nu, \mathcal{F}, Y)\right\}
$$

since the two spaces are not strictly speaking equal. Further, the span $\left\langle\varphi_{x}: x \in X\right\rangle$ is dense in $\mathcal{H}_{1}^{\nu}$ and $\mathcal{H}_{1}^{\tilde{\nu}}$, thus the notation $\operatorname{Co}(\tilde{\nu}, \mathcal{F}, Y)=\operatorname{Co}(\nu, \mathcal{F}, Y)$ is justified.

Regarding the applicability of the theory, it is important to decide whether a given analyzing frame $\mathcal{F}=\left\{\varphi_{x}\right\}_{x \in X}$ has property $F(\nu, Y)$. In the classical theory, where $X$ is a group, the frame is of the special form $\varphi_{x}=\pi(x) g$, where $\pi$ is a group representation and $g \in \mathcal{H}$ a suitable vector. In this case properties of $\mathcal{F}$ break down to properties of the analyzing vector $g$, and it suffices to check admissibility of $g$, see [21, 22, 32, For the continuous wavelet transform concrete conditions can be formulated in terms of smoothness, decay and vanishing moments, generalized in [29] to wavelets over general dilation groups. In our general setup the algebras $\mathcal{A}_{m_{\nu}}$ and $\mathcal{B}_{Y, m_{\nu}}$ embody the concept of admissibility and for the (inhomogeneous) wavelet transform utilized in Section 4 also concrete conditions can be deduced, see e.g. [50].

Concerning the independence of $\operatorname{Co}(\mathcal{F}, Y)$ on the reservoir $\left(\mathcal{H}_{1}^{\nu}\right)^{\urcorner}$we state [50, Lem. 3.7], whose proof carries over directly.

Lemma 2.28. Assume that the analyzing frame $\mathcal{F}$ satisfies $F(\nu, Y)$ and let $S$ be a topological vector space such that $\mathcal{F} \subset S \hookrightarrow \mathcal{H}_{1}^{\nu}$. In case $\mathcal{F}$ is total in $S$ and the reproducing formula $V_{\mathcal{F}} f=R_{\mathcal{F}}\left(V_{\mathcal{F}} f\right)$ extends to all $f \in S^{\urcorner}$(the topological anti-dual of $S$ ) then

$$
\left.\operatorname{Co}(\mathcal{F}, Y)=\{f \in S\urcorner: V_{\mathcal{F}} f \in Y\right\} .
$$


We have the following result concerning the coincidence of the two spaces $\operatorname{Co}(\mathcal{F}, Y)$ and $\operatorname{Co}(\mathcal{G}, Y)$, where $\mathcal{F}$ and $\mathcal{G}$ are two different continuous frames.

Lemma 2.29. Assume that the frames $\mathcal{G}=\left\{g_{x}\right\}_{x \in X}$ and $\mathcal{F}=\left\{f_{x}\right\}_{x \in X}$ satisfy $F(\nu, Y)$. If the Gramian kernels $G[\mathcal{F}, \mathcal{G}]$ and $G[\mathcal{G}, \mathcal{F}]$ defined in (2.9) are both contained in $\mathcal{B}_{Y, m_{\nu}}$, we have $\mathrm{Co}(\mathcal{F}, Y)=\operatorname{Co}(\mathcal{G}, Y)$ in the sense of equivalent quasi-norms.

Proof. This is a consequence of the relations $V_{\mathcal{F}}=G[\mathcal{F}, \mathcal{G}] V_{\mathcal{G}}$ and $V_{\mathcal{G}}=G[\mathcal{G}, \mathcal{F}] V_{\mathcal{F}}$. In view of Lemma 2.14 and Lemma 2.32 we have $V_{\mathcal{G}} f_{x} \in L_{1}^{\nu}(X)$ for a.e. $x \in X$. Further, $V_{\mathcal{G}} f \in L_{\infty}^{1 / \nu}(X)$ for $f \in\left(\mathcal{H}_{1}^{\nu}\right)^{\urcorner}$and hence with Lemma 2.17

$$
V_{\mathcal{F}} f(x)=\left\langle f, f_{x}\right\rangle_{\left.\left(\mathcal{H}_{1}^{\nu}\right)\right\urcorner \times \mathcal{H}_{1}^{\nu}}=\left\langle V_{\mathcal{G}} f, V_{\mathcal{G}} f_{x}\right\rangle_{L_{\infty}^{1 / \nu} \times L_{1}^{\nu}}=\int_{X}\left\langle f, g_{y}\right\rangle \overline{\left\langle g_{y}, f_{x}\right\rangle} d \mu(y)=G[\mathcal{F}, \mathcal{G}] V_{\mathcal{G}} f(x) .
$$

This proves $V_{\mathcal{F}}=G[\mathcal{F}, \mathcal{G}] V_{\mathcal{G}}$, and by symmetry also $V_{\mathcal{G}}=G[\mathcal{G}, \mathcal{F}] V_{\mathcal{F}}$.

It is essential for the theory that the reproducing formula carries over to $\mathrm{Co}(Y)$, which is an immediate consequence of Lemma 2.21

Lemma 2.30. A function $F \in Y$ is of the form $V$ f for some $f \in \operatorname{Co}(Y)$ if and only if $F=R(F)$.

The reproducing formula is the key to prove the main theorem of this section, which corresponds to [24, Prop. 3.7]. We explicitly state the continuitiy of the embedding $\operatorname{Co}(Y) \hookrightarrow\left(\mathcal{H}_{1}^{\nu}\right)^{\urcorner}$.

Theorem 2.31. (i) The space $(\mathrm{Co}(Y),\|\cdot \mid \mathrm{Co}(Y)\|)$ is a quasi-Banach space with quasi-norm constant $C_{Y}$, which is continuously embedded into $\left(\mathcal{H}_{1}^{\nu}\right)^{\urcorner}$.

(ii) The map $V: \operatorname{Co}(Y) \rightarrow Y$ establishes an isometric isomorphism between $\operatorname{Co}(Y)$ and the closed subspace $R(Y)$ of $Y$.

(iii) The map $R: Y \rightarrow Y$ is a projection of $Y$ onto $R(Y)=V(\operatorname{Co}(Y))$.

Proof. In general, we refer to the proof of [24, Prop. 3.7]. However, the continuity of the embedding $\mathrm{Co}(Y) \hookrightarrow\left(\mathcal{H}_{1}^{\nu}\right)^{\urcorner}$is not proved there. It is a consequence of the following estimate for $f \in \operatorname{Co}(Y)$, where Lemma 2.18 is used,

$$
\left\|f\left|\left(\mathcal{H}_{1}^{\nu}\right)^{\urcorner}\|=\| V f\right| L_{\infty}^{1 / \nu}\right\| \leqslant\left\|R\left|Y \rightarrow L_{\infty}^{1 / \nu}\|\| V f\right| Y\right\|=\left\|R\left|Y \rightarrow L_{\infty}^{1 / \nu}\|\| f\right| \operatorname{Co}(Y)\right\| .
$$

Further, the proof of [24, Prop. 3.7] implicitly relies on the validity of $R \circ R=R$ on $Y$, which a-priori is only clear for $L_{2}(X)$. Therefore, we include a proof of this relation here. Let $F \in Y$ and choose compact subsets $\left(K_{n}\right)_{n \in \mathbb{N}}$ with $X=\bigcup_{n \in \mathbb{N}} K_{n}$ and $K_{n} \subset K_{m}$ for $n \leqslant m$, which is possible since $X$ is $\sigma$-compact. Then we define the sets $U_{n}:=\left\{x \in K_{n}:|F(x)| \leqslant n\right\}$, which are relatively compact and thus of finite measure. As a consequence, $F_{n}:=\chi_{U_{n}} F \in L_{2}(X)$. Moreover, $F_{n} \in Y$ since $\left|F_{n}(x)\right| \leqslant|F(x)|$ for every $x \in X$. Since by assumption $R: Y \rightarrow Y$ is well-defined the assignment $y \mapsto|R(x, y) F(y)|$ is integrable for a.e. $x \in X$. As $F_{n}(y) \rightarrow F(y)$ pointwise, Lebesgue's dominated convergence theorem thus yields for these $x \in X$

$$
R F_{n}(x)=\int_{X} R(x, y) F_{n}(y) d \mu(y) \rightarrow \int_{X} R(x, y) F(y) d \mu(y)=R F(x) .
$$

Next, observe that the function $|R(x, \cdot)| m_{\nu}(x, \cdot)$ is integrable for a.e. $x \in X$ since $R \in \mathcal{A}_{m_{\nu}}$. Further, due to $R(Y) \hookrightarrow L_{\infty}^{1 / \nu}(X)$ the following estimate holds true for a.e. $x, y \in X$

$$
\left|R(x, y) R F_{n}(y)\right| \leqslant C|R(x, y)| \nu(y)\left\|F_{n}\left|Y\left\|\leqslant C|R(x, y)| m_{\nu}(x, y) \nu(x)\right\| F\right| Y\right\| .
$$


Another application of Lebesgue's dominated convergence therefore yields for a.e. $x \in X$

$$
R\left(R F_{n}\right)(x)=\int_{X} R(x, y) R F_{n}(y) d \mu(y) \rightarrow \int_{X} R(x, y) R F(y) d \mu(y)=R(R F)(x) .
$$

Since $F_{n} \in L_{2}(X)$ we have $R F_{n}=R\left(R F_{n}\right)$ for every $n \in \mathbb{N}$. Altogether, we obtain

$$
R(R F)(x) \leftarrow R\left(R F_{n}\right)(x)=R F_{n}(x) \rightarrow R F(x) .
$$

Let us finally provide some trivial examples, also given in [24, Cor. 3.8].

Lemma 2.32. If the analyzing frame $\mathcal{F}$ satisfies condition (2.10) for a weight $\nu \geqslant 1$, it has properties $F\left(\nu, L_{2}\right), F\left(\nu, L_{\infty}^{1 / \nu}\right), F\left(\nu, L_{1}^{\nu}\right)$, and it holds

$$
\left.\left(\mathcal{H}_{1}^{\nu}\right)\right\urcorner=\operatorname{Co}\left(\mathcal{F}, L_{\infty}^{1 / \nu}\right), \quad \mathcal{H}_{1}^{\nu}=\operatorname{Co}\left(\mathcal{F}, L_{1}^{\nu}\right), \quad \mathcal{H}=\operatorname{Co}\left(\mathcal{F}, L_{2}\right) .
$$

Typically, the theory cannot be applied if the QBF-space $Y$ is not embedded in $L_{1}^{\text {loc }}(X)$, since then the kernel conditions concerning operations on $Y$ can usually not be fulfilled. Let us close this paragraph with a short discussion of how to proceed in case $Y \nrightarrow L_{1}^{\text {loc }}(X)$.

\section{The case $Y \nrightarrow L_{1}^{\text {loc }}(X)$}

The main idea is to replace $Y$ with a suitable subspace $Z$, which is embedded into $L_{1}^{\text {loc }}(X)$ and fits into the existing theory. The basic observation behind this is that not all the information of $Y$ is used in the definition of the coorbit. In fact, the information about $\mathrm{Co}(Y)$ is fully contained in the subspace $R(Y)$, i.e., we have $\operatorname{Co}(\mathcal{F}, Y)=\operatorname{Co}(\mathcal{F}, R(Y))$. Thus, we can painlessly pass over to a solid subspace $Z$ of $Y$ and regain the same coorbit if

$$
R(Y) \hookrightarrow Z \hookrightarrow Y
$$

This observation motivates the idea to substitute $Y$ - in case $Y$ is not embedded into $L_{1}^{\text {loc }}(X)$ itself - by a suitable subspace $Z$ of $Y$ consisting of locally integrable functions, and then to consider the coorbit of $Z$ instead. In the classical group setting [48] Wiener amalgams [18, 49] were used as suitable substitutes. Since Wiener amalgams rely on the underlying group structure, they cannot be used in our general setup however. Instead, it is possible to resort to the closely related decomposition spaces due to Feichtinger and Gröbner [20], which can be viewed as discrete analoga of Wiener amalgams. This approach has been worked out in [53, where the decomposition space $\mathcal{D}(Y, \mathcal{U})$ with local component $L_{\infty}$ and global component $Y$ is used. It is defined as follows.

Definition 2.33 ([53]). The decomposition space $\mathcal{D}(Y, \mathcal{U})$ associated to a rich solid QBF-space $Y$ on $X$ and an admissible covering $\mathcal{U}=\left\{U_{i}\right\}_{i \in I}$ of $X$ is defined by

$$
\mathcal{D}(Y, \mathcal{U}):=\left\{f \in L_{\infty}^{\mathrm{loc}}(X):\left\|f \left|\mathcal { D } ( Y , \mathcal { U } ) \| : = \| \sum _ { i \in I } \left\|f\left|L_{\infty}\left(U_{i}\right)\left\|\chi_{U_{i}} \mid Y\right\|<\infty\right\} .\right.\right.\right.\right.
$$

Note that the sum $\sum_{i \in I}\|f\|_{L_{\infty}\left(U_{i}\right)} \chi_{U_{i}}$ is locally finite and defines pointwise a function on $X$. 
The space $\mathcal{D}(Y, \mathcal{U})$ is a subspace of $Y$, continuously embedded, and a rich solid QBF-space with the same quasi-norm constant $C_{Y}$ as $Y$. Moreover, it is contained in $L_{1}^{\text {loc }}(X)$ even if $Y$ itself is not. In fact, we have the embedding $\mathcal{D}(Y, \mathcal{U}) \hookrightarrow L_{\infty}^{1 / \omega}(X)$, where $\omega: X \rightarrow(0, \infty)$ defined by $\omega(x):=\max _{i: x \in U_{i}}\left\{\left\|\chi_{U_{i}} \mid Y\right\|^{-1}\right\}$ is a locally bounded weight. For a short proof, let $K \subset X$ be compact and $\left\{U_{i}\right\}_{i \in J}$ the finite subfamily of sets in $\mathcal{U}$ intersecting $K$. Then $\omega(x) \leqslant \max _{i \in J}\left\{\left\|\chi_{U_{i}} \mid Y\right\|^{-1}\right\}$ for all $x \in K$.

In the spirit of [48, Def. 4.1], we may therefore pass over to $\operatorname{Co}(\mathcal{F}, \mathcal{D}(Y, \mathcal{U}))$, the coorbit of $\mathcal{D}(Y, \mathcal{U})$. In general, one can only expect $\operatorname{Co}(\mathcal{F}, \mathcal{D}(Y, \mathcal{U})) \subset\left\{f \in\left(\mathcal{H}_{1}^{\nu}\right)^{\urcorner}: V_{\mathcal{F}} f \in Y\right\}$ and not equality. In many applications however the equality can be proved by methods not available in the abstract setting. Moreover, the choice $\mathcal{D}(Y, \mathcal{U})$ is consistent with the theory due to the result below, which is analogous to a result obtained for Wiener amalgams [48, Thm. 6.1].

Theorem 2.34 (553, Thm. 8.1]). Assume that $Y$ is a rich solid QBF-space and that the analyzing frame $\mathcal{F}$ has property $F(\nu, Y)$. If $\mathcal{U}$ is an admissible covering of $X$ such that the kernel $M_{\mathcal{U}}^{*}=K_{\mathcal{U}}^{*}[\mathcal{F}, \mathcal{F}]$ (defined in (2.13) below) operates continuously on $Y$, then the frame $\mathcal{F}$ has property $F(\nu, \mathcal{D}(Y, \mathcal{U}))$ and it holds

$$
\operatorname{Co}(\mathcal{F}, \mathcal{D}(Y, \mathcal{U}))=\operatorname{Co}(\mathcal{F}, Y)
$$

in the sense of equivalent quasi-norms.

Remark 2.35. The condition that the kernel $M_{\mathcal{U}}^{*}$ operates continuously on $Y$ is fulfilled for instance in the important case when $\mathcal{F}$ has property $D(\delta, \nu, Y)$ (see Definition 2.43 below).

In [53. Thm. 8.1] this theorem was formulated under the additional assumption that $Y$ is continuously embedded into $L_{1}^{\text {loc }}(X)$. However, essential for the proof is only that the frame $\mathcal{F}$ has property $F(\nu, Y)$, wherefore we chose to omit this assumption here.

\subsection{Discretizations}

A main feature of coorbit space theory is its general abstract discretization machinery. With a coorbit characterization of a given function space at hand, the abstract framework (Theorems 2.48 and 2.50 below) provides atomic decompositions of this space, i.e., a representation of functions using "only" a countable number of atoms as building blocks.

Moreover, the function space can be characterized via an equivalent quasi-norm on an associated sequence space.

The transition to sequence spaces bears many advantages, since those usually have a simpler, more accessible structure than the original spaces. For example, the investigation of embedding relations becomes much simpler by performing them on the associated sequence spaces. In addition, atomic decompositions naturally lend themselves to real world representations of the considered functions: By truncation one obtains approximate expansions consisting only of a finite number of atoms.

Our discretization results, Theorem 2.48 and Theorem 2.50, transfer the results from [50, namely Theorem 3.11 and Theorem 3.14, to the general quasi-Banach setting. Applying a different strategy for their proofs, however, we are able to strengthen these results significantly even in the Banach space setting. 


\section{Preliminaries}

Let us introduce the kernel functions $K_{\mathcal{U}}[\mathcal{G}, \mathcal{F}]$ and $K_{\mathcal{U}}^{*}[\mathcal{G}, \mathcal{F}]$, which are related by involution and play a prominent role in the discretization theory. For a family $\mathcal{G}=\left\{\psi_{x}\right\}_{x \in X}$ and an admissible covering $\mathcal{U}=\left\{U_{i}\right\}_{i \in I}$ they are defined by

$$
K_{\mathcal{U}}[\mathcal{G}, \mathcal{F}](x, y):=\sup _{z \in Q_{y}}\left|\left\langle\varphi_{x}, \psi_{z}\right\rangle\right| \quad \text { and } \quad K_{\mathcal{U}}^{*}[\mathcal{G}, \mathcal{F}](x, y):=K_{\mathcal{U}}[\mathcal{G}, \mathcal{F}](y, x)
$$

where $x, y \in X$ and $Q_{y}:=\bigcup_{i: y \in U_{i}} U_{i}$ for $y \in X$. Their mapping properties are essential for two central results, namely Lemmas 2.36 and 2.40, which together with Lemma 2.47 provide the technical foundation for the proofs of Theorem 2.48 and Theorem 2.50 ,

We will subsequently use the symbol $\||\cdot|\|$ for the operator quasi-norm $\|\cdot \mid Y \rightarrow Y\|$ on $Y$.

Lemma 2.36. Let $Y$ be a rich solid $Q B F$-space on $X$ and let the analyzing frame $\mathcal{F}=\left\{\varphi_{x}\right\}_{x \in X}$ possess property $F(\nu, Y)$. Further, let $\mathcal{G}=\left\{\psi_{x}\right\}_{x \in X} \subset \mathcal{H}_{1}^{\nu}$ be a family and $\mathcal{U}=\left\{U_{i}\right\}_{i \in I}$ an admissible covering such that $K_{\mathcal{U}}^{*}:=K_{\mathcal{U}}^{*}[\mathcal{G}, \mathcal{F}]$ defines a bounded operator on $Y$. Then for $f \in \operatorname{Co}(\mathcal{F}, Y)$ the function $\sum_{i \in I} \sup _{z \in U_{i}}\left|V_{\mathcal{G}} f(z)\right| \chi_{U_{i}}$ belongs to $Y$ with the estimate

$$
\left\|\sum_{i \in I} \sup _{z \in U_{i}}\left|V_{\mathcal{G}} f(z)\right| \chi_{U_{i}}|Y\|\leqslant \sigma(\mathcal{U})\|| K_{\mathcal{U}}^{*}\right\|\|\| f \mid \operatorname{Co}(\mathcal{F}, Y) \| .
$$

Note that the $\operatorname{sum} \sum_{i \in I} \sup _{z \in U_{i}}\left|V_{\mathcal{G}} f(z)\right| \chi_{U_{i}}$ is locally finite and defined pointwise.

Proof. Using $V_{\mathcal{G}} f=G[\mathcal{G}, \mathcal{F}] V_{\mathcal{F}} f$ we can estimate for $f \in \operatorname{Co}(\mathcal{F}, Y)$ and all $x \in X$

$$
\begin{aligned}
\sup _{z \in Q_{x}}\left|V_{\mathcal{G}} f(z)\right| & =\sup _{z \in Q_{x}}\left|G[\mathcal{G}, \mathcal{F}] V_{\mathcal{F}} f(z)\right| \leqslant \sup _{z \in Q_{x}} \int|G[\mathcal{G}, \mathcal{F}](z, y)|\left|V_{\mathcal{F}} f(y)\right| d \mu(y) \\
& \leqslant \int \sup _{z \in Q_{x}}|G[\mathcal{G}, \mathcal{F}](z, y)|\left|V_{\mathcal{F}} f(y)\right| d \mu(y) \\
& =\int K_{\mathcal{U}}^{*}[\mathcal{G}, \mathcal{F}](x, y)\left|V_{\mathcal{F}} f(y)\right| d \mu(y)=K_{\mathcal{U}}^{*}[\mathcal{G}, \mathcal{F}]\left(\left|V_{\mathcal{F}} f\right|\right)(x) .
\end{aligned}
$$

For functions $F: X \rightarrow \mathbb{C}$ we further have the estimate

$$
\sup _{z \in Q_{x}}|F(z)| \leqslant \sum_{i \in I} \sup _{z \in U_{i}}|F(z)| \chi_{U_{i}}(x) \leqslant \sigma(\mathcal{U}) \sup _{z \in Q_{x}}|F(z)|,
$$

where $\sigma(\mathcal{U})$ is the intersection number of $\mathcal{U}$. Choosing $F=V_{\mathcal{G}} f$ in (2.14), we can conclude

$$
\left\|\sum_{i \in I} \sup _{z \in U_{i}}\left|V_{\mathcal{G}} f(z)\right| \chi_{U_{i}}|Y\|\leqslant \sigma(\mathcal{U})\|| K_{\mathcal{U}}^{*}\right\|\left|\left\|V _ { \mathcal { F } } f \left|Y\left\|=\sigma(\mathcal{U})\left|\left\|K_{\mathcal{U}}^{*}\right\|\|\| f\right| \operatorname{Co}(\mathcal{F}, Y)\right\|\right.\right.\right.
$$

We can immediately deduce an important result, which corresponds to [50, Lemma 3.12], concerning the sampling of $V_{\mathcal{G}} f$.

Corollary 2.37. With the same assumptions as in the previous lemma let $\left\{x_{i}\right\}_{i \in I}$ be a family of points such that $x_{i} \in U_{i}$. Then $\left\{V_{\mathcal{G}} f\left(x_{i}\right)\right\}_{i \in I} \in Y^{b}(\mathcal{U})$ and it holds

$$
\left\|\left\{V_{\mathcal{G}} f\left(x_{i}\right)\right\}_{i \in I}\left|Y^{b}\|=\| \sum_{i \in I}\right| V_{\mathcal{G}} f\left(x_{i}\right)\left|\chi_{U_{i}}\right| Y\right\| \leqslant \sigma(\mathcal{U})\left\|K_{\mathcal{U}}^{*}\right\|\|\| f \mid \operatorname{Co}(\mathcal{F}, Y) \| .
$$


Let us turn to the synthesis side. Here the following lemma is a key result, which generalizes [24, Lem. 5.10] and whose short direct proof is new and avoids technical difficulties. In particular, it does not rely on [24, Lem. 5.4].

Lemma 2.38. Let $Y$ be a rich solid $Q B F$-space on $X$ and let the analyzing frame $\mathcal{F}=\left\{\varphi_{x}\right\}_{x \in X}$ possess property $F(\nu, Y)$. Further, let $\mathcal{G}=\left\{\psi_{x}\right\}_{x \in X}$ be a family in $\mathcal{H}$ and $\mathcal{U}=\left\{U_{i}\right\}_{i \in I}$ an admissible covering such that $K_{\mathcal{U}}:=K_{\mathcal{U}}[\mathcal{G}, \mathcal{F}]$ defines a bounded operator on $Y$. Then for $\left\{\lambda_{i}\right\}_{i \in I} \in Y^{\natural}(\mathcal{U})$ and for points $x_{i} \in U_{i}$ the series $\sum_{i \in I} \lambda_{i} V_{\mathcal{F}} \psi_{x_{i}}(x)$ converges absolutely for a.e. $x \in X$ defining a function in $Y$ with

$$
\left\|\sum_{i \in I} \lambda_{i} V_{\mathcal{F}} \psi_{x_{i}}\left|Y\|\leqslant\| K_{\mathcal{U}}\|\|\left\{\lambda_{i}\right\}_{i \in I}\right| Y^{\natural}\right\|
$$

If the finite sequences are dense in $Y^{\natural}(\mathcal{U})$ the series also converges unconditionally in the quasinorm of $Y$.

Proof. We have for every $x \in X$ the estimate

$$
\begin{gathered}
\sum_{i \in I}\left|\lambda_{i}\right|\left|V_{\mathcal{F}} \psi_{x_{i}}(x)\right| \leqslant \sum_{i \in I} \mu\left(U_{i}\right)^{-1}\left|\lambda_{i}\right| \int_{X} \chi_{U_{i}}(y) K_{\mathcal{U}}(x, y) d \mu(y) \\
=\int_{X} \sum_{i \in I} \mu\left(U_{i}\right)^{-1}\left|\lambda_{i}\right| \chi_{U_{i}}(y) K_{\mathcal{U}}(x, y) d \mu(y)=K_{\mathcal{U}}\left(\sum_{i \in I} \mu\left(U_{i}\right)^{-1}\left|\lambda_{i}\right| \chi_{U_{i}}\right)(x),
\end{gathered}
$$

where summation and integration can be interchanged due to monotone convergence. Since $\left\{\lambda_{i}\right\}_{i \in I} \in Y^{\natural}$ the sum $\sum_{i \in I} \mu\left(U_{i}\right)^{-1}\left|\lambda_{i}\right| \chi_{U_{i}}$ defines pointwise a function in $Y$. By assumption $K_{\mathcal{U}}$ operates continuously on $Y$ and hence also $K_{\mathcal{U}}\left(\sum_{i \in I} \mu\left(U_{i}\right)^{-1}\left|\lambda_{i}\right| \chi_{U_{i}}\right) \in Y$, which implies $\left|K_{\mathcal{U}}\left(\sum_{i \in I} \mu\left(U_{i}\right)^{-1}\left|\lambda_{i}\right| \chi_{U_{i}}\right)(x)\right|<\infty$ for a.e. $x \in X$. It follows that $\sum_{i \in I} \lambda_{i} V_{\mathcal{F}} \psi_{x_{i}}(x)$ converges absolutely at these points. As a consequence of the solidity of $Y$ and the pointwise estimate

$$
\left|\sum_{i \in I} \lambda_{i} V_{\mathcal{F}} \psi_{x_{i}}\right| \leqslant \sum_{i \in I}\left|\lambda_{i}\right|\left|V_{\mathcal{F}} \psi_{x_{i}}\right| \leqslant K_{\mathcal{U}}\left(\sum_{i \in I} \mu\left(U_{i}\right)^{-1}\left|\lambda_{i}\right| \chi_{U_{i}}\right) \in Y,
$$

the measurable functions $\sum_{i \in I} \lambda_{i} V_{\mathcal{F}} \psi_{x_{i}}$ and $\sum_{i \in I}\left|\lambda_{i}\right|\left|V_{\mathcal{F}} \psi_{x_{i}}\right|$ belong to $Y$ with

$$
\left\|\sum_{i \in I} \lambda_{i} V_{\mathcal{F}} \psi_{x_{i}}\left|Y\|\leqslant\| \sum_{i \in I}\right| \lambda_{i}|| V_{\mathcal{F}} \psi_{x_{i}}|| Y\right\| \leqslant\left\|K_{\mathcal{U}}\right\|\|\|\left\{\lambda_{i}\right\}_{i \in I} \mid Y^{\natural} \| .
$$

It remains to show that $\sum_{i \in I} \lambda_{i} V_{\mathcal{F}} \psi_{x_{i}}$ converges unconditionally in $Y$ to its pointwise limit, if the finite sequences are dense in $Y^{\natural}(\mathcal{U})$. For this we fix an arbitrary bijection $\sigma: \mathbb{N} \rightarrow I$ and obtain as in (2.16)

$$
\left\|\sum_{m=n+1}^{\infty} \lambda_{\sigma(m)} V_{\mathcal{F}} \psi_{x_{\sigma(m)}}\left|Y\|\leqslant\| K_{\mathcal{U}}\|\| \Lambda-\Lambda_{n}^{\sigma}\right| Y^{\natural}\right\|,
$$

where the sequence $\Lambda_{n}^{\sigma}$ is given as in Lemma 2.11. According to this lemma the right-hand side of (2.17) tends to zero for $n \rightarrow \infty$, which finishes the proof.

Corollary 2.39. With the assumptions of the previous lemma $\mathcal{G}=\left\{\psi_{x}\right\}_{x \in X} \subset \operatorname{Co}(\mathcal{F}, Y)$. 
Proof. For every $x \in X$ there is an index $i_{0} \in I$ such that $x \in U_{i_{0}}$. Set $x_{i_{0}}:=x$ and choose arbitrary points $x_{i} \in U_{i}$ for $i \in I \backslash\left\{i_{0}\right\}$. Let $\delta^{i_{0}}$ denote the sequence, which has entry 1 at position $i_{0}$ and is zero elsewhere. Since $Y$ is assumed to be rich $\delta^{i_{0}} \in Y^{\natural}$ and by the previous lemma $V_{\mathcal{F}} \psi_{x}=\sum_{i \in I} \delta_{i}^{i_{0}} V_{\mathcal{F}} \psi_{x_{i}} \in Y$, whence $\psi_{x} \in \operatorname{Co}(\mathcal{F}, Y)$.

The correspondence principle allows to cast Lemma 2.38 in a different form, which corresponds to [50, Lem. 3.11]. However, due to the different deduction the technical assumption $Y^{\natural} \hookrightarrow\left(L_{\infty}^{1 / \nu}\right)^{\natural}$ is not required any more.

Lemma 2.40. With the same assumptions as in Lemma 2.38 the series $\sum_{i \in I} \lambda_{i} \psi_{x_{i}}$ converges unconditionally in the weak ${ }^{*}$-topology of $\left(\mathcal{H}_{1}^{\nu}\right)^{\urcorner}$to an element $f \in \operatorname{Co}(\mathcal{F}, Y)$ with

$$
V_{\mathcal{F}} f=V_{\mathcal{F}}\left(\sum_{i \in I} \lambda_{i} \psi_{x_{i}}\right)=\sum_{i \in I} \lambda_{i} V_{\mathcal{F}} \psi_{x_{i}}
$$

and the estimate $\quad\left\|f\left|\operatorname{Co}(\mathcal{F}, Y)\|=\| \sum_{i \in I} \lambda_{i} \psi_{x_{i}}\right| \operatorname{Co}(\mathcal{F}, Y)\right\| \leqslant\left\|K_{\mathcal{U}}\right\|\left\|\left\{\lambda_{i}\right\}_{i \in I} \mid Y^{\natural}\right\|$.

Moreover, if the finite sequences are dense in $Y^{\natural}(\mathcal{U})$ the series also converges unconditionally in the quasi-norm of $\operatorname{Co}(\mathcal{F}, Y)$.

Proof. If the subset $J \subset I$ is finite we have $V_{\mathcal{F}}\left(\sum_{i \in J} \lambda_{i} \psi_{x_{i}}\right)(x)=\sum_{i \in J} \lambda_{i} V_{\mathcal{F}} \psi_{x_{i}}(x)$ for all $x \in X$. Moreover, we have proved in Lemma 2.38 that $\sum_{i \in I} \lambda_{i} V_{\mathcal{F}} \psi_{x_{i}}$ converges pointwise absolutely a.e. to a function in $Y$. In order to apply the correspondence principle, Corollary 2.23, it remains to verify that the sums $\sum_{i \in J} \lambda_{i} \psi_{x_{i}}$ for finite subsets $J \subset I$ are uniformly bounded in $\left(\mathcal{H}_{1}^{\nu}\right)^{\urcorner}$. With the continuous embedding $\mathrm{Co}(Y) \hookrightarrow\left(\mathcal{H}_{1}^{\nu}\right)^{\urcorner}$from Theorem 2.31 we can conclude

$$
\left\|\sum_{i \in J} \lambda_{i} \psi_{x_{i}}\left|\left(\mathcal{H}_{1}^{\nu}\right)^{\urcorner}\|\lesssim\| \sum_{i \in J} \lambda_{i} \psi_{x_{i}}\right| \operatorname{Co}(Y)\right\|=\left\|\sum_{i \in J} \lambda_{i} V_{\mathcal{F}} \psi_{x_{i}}\left|Y\|\leqslant\| \sum_{i \in I}\right| \lambda_{i}|| V_{\mathcal{F}} \psi_{x_{i}}|| Y\right\|
$$

for every finite subset $J \subset I$, where we used that $\psi_{x_{i}} \in \operatorname{Co}(Y)$ for all $i \in I$ by Corollary 2.39, We have shown in the proof of Lemma 2.38 that $\sum_{i \in I}\left|\lambda_{i}\right|\left|V_{\mathcal{F}} \psi_{x_{i}}\right|$ is a function in $Y$. Hence the sums are uniformly bounded in $\left(\mathcal{H}_{1}^{\nu}\right)^{\urcorner}$and Corollary 2.23 implies the unconditional weak*convergence of $\sum_{i \in I} \lambda_{i} \psi_{x_{i}}$ to an element $\left.f \in\left(\mathcal{H}_{1}^{\nu}\right)\right\urcorner$. Moreover, $f \in \operatorname{Co}(Y)$ because Corollary 2.23 together with the previous lemma asserts that $V_{\mathcal{F}} f=\sum_{i \in I} \lambda_{i} V_{\mathcal{F}} \psi_{x_{i}} \in Y$.

It remains to show that $\sum_{i \in I} \lambda_{i} \psi_{x_{i}}$ converges unconditionally in $\operatorname{Co}(\mathcal{F}, Y)$, if the finite sequences are dense in $Y^{\natural}$. For a subset $\tilde{I} \subset I$ let $\tilde{\Lambda}$ denote the sequence which coincides with $\Lambda$ on $\tilde{I}$ and is trivial elsewhere. By solidity $\tilde{\Lambda} \in Y^{\natural}$ and - applying what we have proved so far - the sum $\sum_{i \in \tilde{I}} \lambda_{i} \psi_{x_{i}}$ converges in the weak*-topology to an element of $\mathrm{Co}(Y)$ and $V_{\mathcal{F}}\left(\sum_{i \in \tilde{I}} \lambda_{i} \psi_{x_{i}}\right)=\sum_{i \in \tilde{I}} \lambda_{i} V_{\mathcal{F}} \psi_{x_{i}}$. In view of (2.17) we conclude

$$
\left\|\sum_{m=n+1}^{\infty} \lambda_{\sigma(m)} \psi_{x_{\sigma(m)}}\left|\operatorname{Co}(Y)\|=\| \sum_{m=n+1}^{\infty} \lambda_{\sigma(m)} V_{\mathcal{F}} \psi_{x_{\sigma(m)}}\right| Y\right\| \rightarrow 0 \quad(n \rightarrow \infty),
$$

for an arbitrary bijection $\sigma: \mathbb{N} \rightarrow I$, which finishes the proof.

\section{Atomic decompositions}

Our first goal is to obtain atomic decompositions of the coorbit $\mathrm{Co}(Y)$. Since $\mathrm{Co}(Y)$ is isometrically isomorphic to the function space $R(Y)$ we initially focus on this space and recall from 
Theorem 2.31 that for functions $F \in R(Y)$ the reproducing formula holds, i.e.

$$
F=R(F)=\int_{X} F(y) R(\cdot, y) d \mu(y), \quad F \in R(Y) .
$$

This identity can be interpreted as a "continuous atomic decomposition" of $F$ with atoms $R(\cdot, y)$ indexed by $y \in X$. The strategy is to discretize the integral, an approach which originates from Feichtinger and Gröchenig [22] and was also used in subsequent papers e.g. in [24, 50]. To this end let $\mathcal{U}=\left\{U_{i}\right\}_{i \in I}$ be an admissible covering of $X$ and let $\Phi=\left\{\Phi_{i}\right\}_{i \in I}$ be a $\mathcal{U}$-PU, i.e. a partition of unity subordinate to the covering $\mathcal{U}$ consisting of measurable functions $\Phi_{i}$ which satisfy

(i) $0 \leqslant \Phi_{i}(x) \leqslant 1$ for all $x \in X$ and all $i \in I$,

(ii) $\operatorname{supp} \Phi_{i} \subset U_{i}$ for all $i \in I$,

(iii) $\sum_{i \in I} \Phi_{i}(x)=1$ for all $x \in X$.

We note that the construction of such a family $\Phi$ with respect to a locally finite covering is standard, see e.g. [23, p. 127]. Using $\Phi$ the integral operator $R$ can be written in the form

$$
R(F)(x)=\sum_{i \in I} \int_{X} \Phi_{i}(y) F(y) R(x, y) d \mu(y) .
$$

A formal discretization yields a discrete integral operator $U_{\Phi}$, called the discretization operator,

$$
U_{\Phi} F(x):=\sum_{i \in I} c_{i} F\left(x_{i}\right) R\left(x, x_{i}\right)
$$

where $c_{i}:=\int_{X} \Phi_{i}(y) d \mu(y)$ and the points $\left\{x_{i}\right\}_{i \in I}$ are chosen such that $x_{i} \in U_{i}$. Here we must give meaning to the point evaluations $F\left(x_{i}\right)$ since in general $F \in Y$ only determines an equivalence class of functions where point evaluations are not well-defined. However, the operator $U_{\Phi}$ is only applied to elements $F \in R(Y)$ and pointwise evaluation can be understood in the sense

$$
F\left(x_{i}\right)=(R F)\left(x_{i}\right)=\int_{X} R\left(x_{i}, y\right) F(y) d \mu(y) .
$$

Intuitively, $U_{\Phi} F$ approximates $R(F)$ because the discretization resembles a Riemannian sum of the integral. Hence we can hope to obtain an atomic decomposition from the relation

$$
F=R(F) \approx U_{\Phi} F=\sum_{i \in I} c_{i} F\left(x_{i}\right) R\left(\cdot, x_{i}\right) .
$$

So far our considerations were just formal. To make the argument precise we have to impose conditions on $\mathcal{F}$ so that $U_{\Phi}$ is a well-defined operator. It turns out that here mapping properties of the kernels $M_{\mathcal{U}}:=K_{\mathcal{U}}[\mathcal{F}, \mathcal{F}]$ and $M_{\mathcal{U}}^{*}:=K_{\mathcal{U}}^{*}[\mathcal{F}, \mathcal{F}]$ come into play. Recalling the definition (2.13) of $K_{\mathcal{U}}, K_{\mathcal{U}}^{*}$ we have for $x, y \in X$

$$
M_{\mathcal{U}}(x, y)=\sup _{z \in Q_{y}}\left|\left\langle\varphi_{x}, \varphi_{z}\right\rangle\right| \quad \text { and } \quad M_{\mathcal{U}}^{*}(x, y)=M_{\mathcal{U}}(y, x)
$$

with $Q_{y}=\bigcup_{i: y \in U_{i}} U_{i}$ for the covering $\mathcal{U}=\left\{U_{i}\right\}_{i \in I}$.

The lemma below provides definition (2.18) with a solid foundation. 
Lemma 2.41. If $M_{\mathcal{U}}$ and $M_{\mathcal{U}}^{*}$ given in (2.19) are bounded operators on $Y$ the discretization operator defined in (2.18) is a well-defined continuous operator $U_{\Phi}: R(Y) \rightarrow R(Y)$ with operator quasi-norm $\left\|U_{\Phi}\left|R(Y) \rightarrow R(Y)\|\leqslant \sigma(\mathcal{U})\| M_{\mathcal{U}}\|\|\left\|M_{\mathcal{U}}^{*}\right\|\right|\right.$. In general, the convergence of the sum in (2.18) is pointwise absolutely a.e.. If the finite sequences are dense in $Y^{\natural}$ the convergence is also in the quasi-norm of $Y$.

Proof. For $F \in R(Y)$ Lemma 2.30 gives an element $f \in \operatorname{Co}(Y)$ such that $F(x)=V f(x)$ for all $x \in X$. Thus, using Corollary 2.37 with $\mathcal{G}=\mathcal{F}$, we can conclude $\left\{F\left(x_{i}\right)\right\}_{i \in I} \in Y^{b}$ with $\left\|\left\{F\left(x_{i}\right)\right\}_{i \in I}\left|Y^{b}\|\leqslant \sigma(\mathcal{U})\| M_{\mathcal{U}}^{*}\|\|\|F \mid Y\|\right.\right.$. Since $\lambda_{i} \mapsto \mu\left(U_{i}\right) \lambda_{i}$ is an isometry from $Y^{b}$ to $Y^{\natural}$ and since $0 \leqslant c_{i} \leqslant \mu\left(U_{i}\right)$ for all $i \in I$ it follows $\left\{c_{i} F\left(x_{i}\right)\right\}_{i \in I} \in Y^{\natural}(\mathcal{U})$ and $\left\|\left\{c_{i} F\left(x_{i}\right)\right\}_{i \in I} \mid Y^{\natural}\right\| \leqslant$ $\left\|\left\{F\left(x_{i}\right)\right\}_{i \in I} \mid Y^{b}\right\|$. Therefore by Lemma 2.40 the sum $\sum_{i \in I} c_{i} F\left(x_{i}\right) \varphi_{x_{i}}$ converges in the weak*topology to an element in $\mathrm{Co}(Y)$ and $U_{\Phi} F=V\left(\sum_{i \in I} c_{i} F\left(x_{i}\right) \varphi_{x_{i}}\right)$. As a consequence $U_{\Phi} F \in$ $R(Y)$ and again with Lemma 2.40

$$
\begin{aligned}
\left\|U_{\Phi} F \mid Y\right\| & =\left\|\sum_{i \in I} c_{i} F\left(x_{i}\right) \varphi_{x_{i}} \mid \operatorname{Co}(Y)\right\| \\
& \leqslant\left\|M_{\mathcal{U}}\right\|\left\|\left\{F\left(x_{i}\right)\right\}_{i \in I}\left|Y^{b}\|\leqslant \sigma(\mathcal{U})\| M_{\mathcal{U}}\|\| M_{\mathcal{U}}^{*}\|\| F\right| Y\right\| .
\end{aligned}
$$

The operator $U_{\Phi}$ is self-adjoint in a certain sense.

Lemma 2.42. Let $\mathcal{U}=\left\{U_{i}\right\}_{i \in I}$ be an admissible covering and assume that the associated maximal kernels $M_{\mathcal{U}}$ and $M_{\mathcal{U}}^{*}$ of the analyzing frame $\mathcal{F}$ belong to $\mathcal{A}_{m_{\nu}}$. Then $U_{\Phi}$ is a welldefined operator on $R\left(L_{\infty}^{1 / \nu}\right)$ and $R\left(L_{1}^{\nu}\right)$ and for every $F \in R\left(L_{\infty}^{1 / \nu}\right)$ and $G \in R\left(L_{1}^{\nu}\right)$ it holds

$$
\left\langle U_{\Phi} F, G\right\rangle_{L_{\infty}^{1 / \nu} \times L_{1}^{\nu}}=\left\langle F, U_{\Phi} G\right\rangle_{L_{\infty}^{1 / \nu} \times L_{1}^{\nu}}
$$

Proof. For $F \in R\left(L_{\infty}^{1 / \nu}\right)$ we have $F(x)=\langle F, R(\cdot, x)\rangle_{L_{\infty}^{1 / \nu} \times L_{1}^{\nu}}$ and - by arguments in the proof of Lemma 2.41 for $Y=L_{\infty}^{1 / \nu}-\left\{c_{i} F\left(x_{i}\right)\right\}_{i \in I} \in\left(L_{\infty}^{1 / \nu}\right)^{\natural}$. Therefore, $\sum_{i \in I} c_{i}\left|F\left(x_{i}\right)\right|\left|R\left(\cdot, x_{i}\right)\right| \in L_{\infty}^{1 / \nu}$ by Lemma 2.38 and (2.15). Analogous statements hold for $G \in R\left(L_{1}^{\nu}\right)$. We conclude

$$
\begin{aligned}
\left\langle U_{\Phi} F, G\right\rangle_{L_{\infty}^{1 / \nu} \times L_{1}^{\nu}} & =\sum_{i \in I} c_{i} F\left(x_{i}\right)\left\langle R\left(\cdot, x_{i}\right), G\right\rangle_{L_{\infty}^{1 / \nu} \times L_{1}^{\nu}}=\sum_{i \in I} c_{i} F\left(x_{i}\right) \overline{G\left(x_{i}\right)} \\
& =\sum_{i \in I} c_{i} \overline{G\left(x_{i}\right)}\left\langle F, R\left(\cdot, x_{i}\right)\right\rangle_{L_{\infty}^{1 / \nu} \times L_{1}^{\nu}}=\left\langle F, U_{\Phi} G\right\rangle_{L_{\infty}^{1 / \nu} \times L_{1}^{\nu}},
\end{aligned}
$$

where Lebesgue's dominated convergence theorem was used.

Our next aim is to find suitable conditions on $\Phi$ and $\mathcal{U}$ such that the discretization operator $U_{\Phi}$ is invertible. The possible expansion

$$
F=U_{\Phi} U_{\Phi}^{-1} F=\sum_{i \in I} c_{i}\left(U_{\Phi}^{-1} F\right)\left(x_{i}\right) R\left(\cdot, x_{i}\right)
$$

then yields an atomic decomposition for $F \in R(Y)$. Intuitively, for the invertibility of $U_{\Phi}$ the functions $F \in R(Y)$ must be sufficiently "smooth", so that a discrete sampling is possible without loss of information. Since $R(Y)$ is the isomorphic image of $\mathrm{Co}(Y)$ under the voice transform, we have to ensure that the transforms $V_{\mathcal{F}} f$ of elements $f \in \operatorname{Co}(Y)$ are smooth enough. An appropriate tool for the control of the smoothness are the oscillation kernels, a 
concept originally due to Feichtinger and Gröchenig. We use the extended definition from 25], utilizing a phase function $\Gamma: X \times X \rightarrow \mathbb{S}^{1}$ where $\mathbb{S}^{1}=\{z \in \mathbb{C}:|z|=1\}$, namely

$$
\operatorname{osc}_{\mathcal{U}, \Gamma}(x, y):=\sup _{z \in Q_{y}}\left|R_{\mathcal{F}}(x, y)-\Gamma(y, z) R_{\mathcal{F}}(x, z)\right| \quad \text { and } \quad \operatorname{osc}_{\mathcal{U}, \Gamma}^{*}(x, y):=\operatorname{osc}_{\mathcal{U}, \Gamma}(y, x)
$$

with $x, y \in X$ and $Q_{y}$ as in (2.13). The choice $\Gamma \equiv 1$ yields the kernels used in [24, 50].

We can now formulate a condition on $\mathcal{F}$ which ensures invertibility of $U_{\Phi}$, but which is weaker than the assumptions made in [24, 50] since we allow a larger class of coverings and weights.

Definition 2.43. We say a tight continuous frame $\mathcal{F}=\left\{\varphi_{x}\right\}_{x \in X} \subset \mathcal{H}$ possesses property $D(\delta, \nu, Y)$ for a weight $\nu \geqslant 1$ and some $\delta>0$ if it has property $F(\nu, Y)$ and if there exists an admissible covering $\mathcal{U}$ and a phase function $\Gamma: X \times X \rightarrow \mathbb{S}^{1}$ so that

(i) $\left|R_{\mathcal{F}}\right|, \operatorname{osc}_{\mathcal{U}, \Gamma}, \operatorname{osc}_{\mathcal{U}, \Gamma}^{*} \in \mathcal{B}_{Y, m_{\nu}}$.

(ii) $\left\|\operatorname{osc}_{\mathcal{U}, \Gamma} \mid \mathcal{B}_{Y, m_{\nu}}\right\|<\delta$ and $\left\|\operatorname{osc}_{\mathcal{U}, \Gamma}^{*} \mid \mathcal{B}_{Y, m_{\nu}}\right\|<\delta$.

Remark 2.44. A frame $\mathcal{F}$ with property $D(\delta, \nu, Y)$ for a covering $\mathcal{U}$ and a phase function $\Gamma$ automatically possesses properties $D\left(\delta, \nu, L_{\infty}^{1 / \nu}\right)$ and $D\left(\delta, \nu, L_{1}^{\nu}\right)$ for the same covering $\mathcal{U}$ and the same phase function $\Gamma$.

Proof. Every $K \in \mathcal{A}_{m_{\nu}}$ operates continuously on $L_{\infty}^{1 / \nu}$ and $L_{1}^{\nu}$ with $\left\|K \mid L_{\infty}^{1 / \nu} \rightarrow L_{\infty}^{1 / \nu}\right\| \leqslant$ $\left\|K \mid \mathcal{A}_{m_{\nu}}\right\|$ and $\left\|K\left|L_{1}^{\nu} \rightarrow L_{1}^{\nu}\|\leqslant\| K\right| \mathcal{A}_{m_{\nu}}\right\|$. Moreover, for $Y=L_{\infty}^{1 / \nu}$ or $Y=L_{1}^{\nu}$ it holds $R(Y) \hookrightarrow$ $L_{\infty}^{1 / \nu}$ and the algebras $\mathcal{B}_{Y, m_{\nu}}$ and $\mathcal{A}_{m_{\nu}}$ coincide with equal norms.

Note that for a measurable kernel function $K: X \times X \rightarrow \mathbb{C}$ the equality $\|K|\|=|\||| K||\|$ does not hold in general. However, we have the following result.

Lemma 2.45. Let $K, L: X \times X \rightarrow \mathbb{C}$ be two measurable kernels and assume that $|K|$ acts continuously on $Y$. Then, if $|L(x, y)| \leqslant|K(x, y)|$ for almost all $x, y \in X$, also $L$ acts continuously on $Y$ with the estimate $\|L\||\leqslant||| K|| \|$. In particular, $K$ acts continuously on $Y$ with ||$K|||\leqslant||||K||| \mid$.

Let us record an important consequence of the previous lemma.

Corollary 2.46. If the frame $\mathcal{F}$ has property $D(\delta, \nu, Y)$ the kernels $R_{\mathcal{F}},\left|R_{\mathcal{F}}\right|, \operatorname{osc}_{\mathcal{U}, \Gamma}, \operatorname{osc}_{\mathcal{U}, \Gamma}^{*}$, $M_{\mathcal{U}}$, and $M_{\mathcal{U}}^{*}$ are continuous operators on $Y$.

Proof. For all $x, y \in X$ we have $\left|R_{\mathcal{F}}(x, y)\right| \leqslant M_{\mathcal{U}}(x, y)$ as well as the estimates

$$
M_{\mathcal{U}}(x, y) \leqslant \operatorname{osc}_{\mathcal{U}, \Gamma}(x, y)+\left|R_{\mathcal{F}}(x, y)\right| \quad \text { and } \quad \operatorname{osc}_{\mathcal{U}, \Gamma}(x, y) \leqslant M_{\mathcal{U}}(x, y)+\left|R_{\mathcal{F}}(x, y)\right| .
$$

The corresponding estimates for the involuted kernels also hold true. Hence Lemma 2.45yields the result.

The following lemma shows that $U_{\Phi} F$ approximates $F \in R(Y)$ if the analyzing frame possesses property $D(\delta, \nu, Y)$ for a suitably small $\delta>0$. It corresponds to [24, Thm. 5.13] and the proof is still valid in our setting - with the triangle inequality replaced by the corresponding quasi-triangle inequality. 
Lemma 2.47. Suppose that the analyzing frame $\mathcal{F}$ possesses property $D(\delta, \nu, Y)$ for some $\delta>0$ with associated covering $\mathcal{U}=\left\{U_{i}\right\}_{i \in I}$ and phase function $\Gamma$. Then the discretization operator $U_{\Phi}$ for some $\mathcal{U}-P U \Phi$ is a well-defined bounded operator $U_{\Phi}: R(Y) \rightarrow R(Y)$ and it holds

$$
\left\|I d-U_{\Phi} \mid R(Y) \rightarrow R(Y)\right\| \leqslant \delta\left(\|R\|+\left\|M_{\mathcal{U}}^{*}\right\|\right) C_{Y} .
$$

Proof. For $F \in R(Y)$ there is $f \in \operatorname{Co}(Y)$ with $F=V f$. By adapting the proof of Lemma 2.36, it can be shown that $\widetilde{H}:=\sum_{i \in I} \sup _{z \in U_{i}}|V f(z)| \Phi_{i} \in Y$ with $\left\|\widetilde{H}\left|Y\|\leqslant\| M_{\mathcal{U}}^{*}\|\|\|f \mid \operatorname{Co}(\mathcal{F}, Y)\|\right.\right.$. The intersection number $\sigma(\mathcal{U})$ does not come into play here, since the inequality (2.14) can be improved when using $\Phi_{i}$ instead of $\chi_{U_{i}}$. A solidity argument yields $H:=\sum_{i \in I}\left|F\left(x_{i}\right)\right| \Phi_{i} \in Y$ and also $\sum_{i \in I} F\left(x_{i}\right) \overline{\Gamma\left(\cdot, x_{i}\right)} \Phi_{i} \in Y$ with respective quasi-norms dominated by $\|\widetilde{H} \mid Y\|$.

Let us introduce the auxiliary operator $S_{\Phi}: R(Y) \rightarrow R(Y)$, given pointwise for $x \in X$ by

$$
S_{\Phi} F(x):=R\left(\sum_{i \in I} F\left(x_{i}\right) \overline{\Gamma\left(\cdot, x_{i}\right)} \Phi_{i}\right)(x) .
$$

Since $F=R(F)$ we can estimate

$$
\left\|F-S_{\Phi} F\left|Y\|=\| R\left(F-\sum_{i \in I} F\left(x_{i}\right) \overline{\Gamma\left(\cdot, x_{i}\right)} \Phi_{i}\right)\right| Y\right\| \leqslant\|R\|\left\|F-\sum_{i \in I} F\left(x_{i}\right) \overline{\Gamma\left(\cdot, x_{i}\right)} \Phi_{i} \mid Y\right\| .
$$

We further obtain for every $x \in X$, because $F(x)=R(F)(x)$ even pointwise,

$$
\begin{aligned}
& \left|F(x)-\sum_{i \in I} F\left(x_{i}\right) \overline{\Gamma\left(x, x_{i}\right)} \Phi_{i}(x)\right|=\left|\sum_{i \in I}\left(R(F)(x)-\overline{\Gamma\left(x, x_{i}\right)} R(F)\left(x_{i}\right)\right) \Phi_{i}(x)\right| \\
& \quad \leqslant \sum_{i \in I} \Phi_{i}(x) \int_{X}\left|R(y, x)-\Gamma\left(x, x_{i}\right) R\left(y, x_{i}\right)\right||F(y)| d \mu(y) \leqslant \operatorname{osc}_{\mathcal{U}, \Gamma}^{*}(|F|)(x) .
\end{aligned}
$$

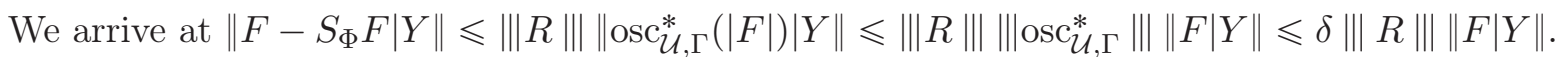
Let us now estimate the difference of $U_{\Phi}$ and $S_{\Phi}$. First we see that for $x \in X$

$$
S_{\Phi} F(x)=\int_{X} R(x, y) \sum_{i \in I} F\left(x_{i}\right) \overline{\Gamma\left(y, x_{i}\right)} \Phi_{i}(y) d \mu(y)=\sum_{i \in I} \int_{X} R(x, y) F\left(x_{i}\right) \overline{\Gamma\left(y, x_{i}\right)} \Phi_{i}(y) d \mu(y) .
$$

Here we used Lebesgue's dominated convergence theorem, which we use again to obtain

$$
\begin{gathered}
\left|U_{\Phi} F(x)-S_{\Phi} F(x)\right|=\left|\sum_{i \in I} \int_{X} \Phi_{i}(y) F\left(x_{i}\right)\left(R\left(x, x_{i}\right)-\overline{\Gamma\left(y, x_{i}\right)} R(x, y)\right) d \mu(y)\right| \\
\leqslant \sum_{i \in I} \int_{X}\left|F\left(x_{i}\right)\right| \Phi_{i}(y) \operatorname{osc}_{\mathcal{U}, \Gamma}(x, y) d \mu(y)=\int_{X} \sum_{i \in I}\left|F\left(x_{i}\right)\right| \Phi_{i}(y) \operatorname{osc}_{\mathcal{U}, \Gamma}(x, y) d \mu(y)=\operatorname{osc}_{\mathcal{U}, \Gamma}(H)(x),
\end{gathered}
$$

where $H=\sum_{i \in I}\left|F\left(x_{i}\right)\right| \Phi_{i}$ as above. We conclude

$$
\left\|U_{\Phi} F-S_{\Phi} F\left|Y\|\leqslant\| \operatorname{osc}_{\mathcal{U}, \Gamma}(H)\right| Y\right\| \leqslant\left\|\operatorname{osc}_{\mathcal{U}, \Gamma}|\|\| H| Y\right\| \leqslant \delta\|\| M_{\mathcal{U}}^{*}\|\|\|F \mid Y\| .
$$

Hence, altogether we have proved

$$
\left\|F-U_{\Phi} F\left|Y\left\|\leqslant C_{Y}\left(\left\|F-S_{\Phi} F\left|Y\|+\| S_{\Phi} F-U_{\Phi} F\right| Y\right\|\right) \leqslant \delta C_{Y}\right\| F\right| Y\right\|\left(\left\|M_{\mathcal{U}}^{*}\right\||+\|\mid R\|) .\right.
$$


If the righthand side of (2.21) is less than one, $U_{\Phi}: R(Y) \rightarrow R(Y)$ is boundedly invertible with the Neumann expansion $U_{\Phi}^{-1}=\sum_{n=0}^{\infty}\left(I d-U_{\Phi}\right)^{n}$, which is still valid in the quasi-Banach setting.

Finally, we are able to prove a cornerstone of the discretization theory, which generalizes [24, Thm. 5.7] and [50, Thm. 3.11]. Note that the characterization via the sequence spaces is a new result even in the Banach case and that we can drop many technical restrictions.

Theorem 2.48. Let $Y$ be a rich solid $Q B F$-space with quasi-norm constant $C_{Y}$ and suppose that the analyzing frame $\mathcal{F}=\left\{\varphi_{x}\right\}_{x \in X}$ possesses property $D(\delta, \nu, Y)$ for the covering $\mathcal{U}=\left\{U_{i}\right\}_{i \in I}$ and a small enough $\delta>0$ such that

$$
\delta\left(\left(1+C_{Y}\right)\left\|\mid R_{\mathcal{F}}\right\| \mathcal{B}_{Y, m_{\nu}} \|+\delta C_{Y}\right) C_{Y} \leqslant 1 .
$$

Choosing arbitrary points $x_{i} \in U_{i}$, the sampled frame $\mathcal{F}_{d}:=\left\{\varphi_{i}\right\}_{i \in I}:=\left\{\varphi_{x_{i}}\right\}_{i \in I}$ then possesses a "dual family" $\widehat{\mathcal{F}}_{d}=\left\{\psi_{i}\right\}_{i \in I} \subset \mathcal{H}_{1}^{\nu} \cap \operatorname{Co}(Y)$ such that the following holds true:

(i) (Analysis) An element $f \in\left(\mathcal{H}_{1}^{\nu}\right)^{\urcorner}$belongs to $\operatorname{Co}(Y)$ if and only if $\left\{\left\langle f, \varphi_{i}\right\rangle\right\}_{i \in I} \in Y^{b}(\mathcal{U})$ (or $\left.\left\{\left\langle f, \psi_{i}\right\rangle\right\}_{i \in I} \in Y^{\natural}(\mathcal{U})\right)$ and we have the quasi-norm equivalences

$$
\left\|f\left|\operatorname{Co}(Y)\|\asymp\|\left\{\left\langle f, \varphi_{i}\right\rangle\right\}_{i \in I}\right| Y^{b}(\mathcal{U})\right\| \quad \text { and } \quad\left\|f\left|\operatorname{Co}(Y)\|\asymp\|\left\{\left\langle f, \psi_{i}\right\rangle\right\}_{i \in I}\right| Y^{\natural}(\mathcal{U})\right\| .
$$

(ii) (Synthesis) For every sequence $\left\{\lambda_{i}\right\}_{i \in I} \in Y^{\natural}(\mathcal{U})$ it holds $f=\sum_{i \in I} \lambda_{i} \varphi_{i} \in \operatorname{Co}(Y)$ with $\left\|f\left|\operatorname{Co}(Y)\|\lesssim\|\left\{\lambda_{i}\right\}_{i \in I}\right| Y^{\natural}(\mathcal{U})\right\|$. In general, the convergence of the sum is in the weak* topology induced by $\left(\mathcal{H}_{1}^{\nu}\right)$. It is unconditional in the quasi-norm of $\mathrm{Co}(Y)$, if the finite sequences are dense in $Y^{\natural}$. Similarly, $f=\sum_{i \in I} \lambda_{i} \psi_{i} \in \operatorname{Co}(Y)$ with $\|f \mid \operatorname{Co}(Y)\| \lesssim$ $\left\|\left\{\lambda_{i}\right\}_{i \in I} \mid Y^{b}(\mathcal{U})\right\|$ in case $\left\{\lambda_{i}\right\}_{i \in I} \in Y^{b}(\mathcal{U})$.

(iii) (Reconstruction) For all $f \in \mathrm{Co}(Y)$ we have $f=\sum_{i \in I}\left\langle f, \psi_{i}\right\rangle \varphi_{i}$ and $f=\sum_{i \in I}\left\langle f, \varphi_{i}\right\rangle \psi_{i}$.

Proof. According to Remark 2.44 the frame $\mathcal{F}$ has properties $D\left(\delta, \nu, L_{1}^{\nu}\right)$ and $D\left(\delta, \nu, L_{\infty}^{1 / \nu}\right)$ with respect to the covering $\mathcal{U}$, and by Lemma 2.32 it holds $\left.\left(\mathcal{H}_{1}^{\nu}\right)\right\urcorner=\operatorname{Co}\left(L_{\infty}^{1 / \nu}\right)$ and $\mathcal{H}_{1}^{\nu}=\operatorname{Co}\left(L_{1}^{\nu}\right)$. In view of Theorem 2.31 the voice transform $\left.V:\left(\mathcal{H}_{1}^{\nu}\right)\right\urcorner \rightarrow R\left(L_{\infty}^{1 / \nu}\right)$ is thus a boundedly invertible operator with isometric restrictions $V: \mathrm{Co}(Y) \rightarrow R(Y)$ and $V: \mathcal{H}_{1}^{\nu} \rightarrow R\left(L_{1}^{\nu}\right)$.

Let us fix a $\mathcal{U}$-PU $\Phi=\left\{\Phi_{i}\right\}_{i \in I}$ and put $c_{i}:=\int_{X} \Phi_{i}(y) d \mu(y)$. According to Lemma 2.42 the corresponding discretization operator $U_{\Phi}$ is well-defined and bounded on $R\left(L_{\infty}^{1 / \nu}\right)$. Condition (2.22) on $\delta$ further implies that $U_{\Phi}: R\left(L_{\infty}^{1 / \nu}\right) \rightarrow R\left(L_{\infty}^{1 / \nu}\right)$ is boundedly invertible as a consequence of Lemma 2.47. Indeed, using the estimates $\left\|\left|M_{\mathcal{U}}^{*} \|\right| \leqslant C_{Y}\left(\left|\left\|\left|R_{\mathcal{F}}\right|\right\|\right|+\|\right.\right.$ osc $\left.\mathcal{U}^{*}, \Gamma \mid \|\right)$ and ||$\left|R_{\mathcal{F}}\right||\leqslant|||\left|R_{\mathcal{F}}\right| \| \mid$ together with the assumption $\| \operatorname{osc}_{\mathcal{U}, \Gamma}^{*}||<\delta$ we can deduce

$$
\begin{aligned}
\delta\left(\left\|\left|R_{\mathcal{F}}\left\|\left|+\left\|M_{\mathcal{U}}^{*}\right\|\right|\right) C_{Y}\right.\right.\right. & \leqslant \delta\left(( 1 + C _ { Y } ) \left\|| | R _ { \mathcal { F } } \left|\left\|\left|+C_{Y}\left\|\mid \operatorname{osc}_{\mathcal{U}, \Gamma}^{*}\right\|\right) C_{Y}\right.\right.\right.\right. \\
& <\delta\left(\left(1+C_{Y}\right)\left\|\mid R_{\mathcal{F}}\right\| \mathcal{B}_{Y, m_{\nu}} \|+C_{Y} \delta\right) C_{Y} \leqslant 1 .
\end{aligned}
$$

Analogously, it follows that $U_{\Phi}: R\left(L_{1}^{\nu}\right) \rightarrow R\left(L_{1}^{\nu}\right)$ and $U_{\Phi}: R(Y) \rightarrow R(Y)$ are boundedly invertible.

For the proof it is useful to note that the operator $T:=V^{-1} U_{\Phi}^{-1} V:\left(\mathcal{H}_{1}^{\nu}\right)^{\urcorner} \rightarrow\left(\mathcal{H}_{1}^{\nu}\right)^{\urcorner}$is a boundedly invertible isomorphism, whose restrictions $T: \mathcal{H}_{1}^{\nu} \rightarrow \mathcal{H}_{1}^{\nu}$ and $T: \operatorname{Co}(Y) \rightarrow \operatorname{Co}(Y)$ 
are also boundedly invertible. Moreover, $T$ is "self-adjoint". For this observe that relation (2.20) also holds for the inverse $U_{\Phi}^{-1}=\sum_{n=0}^{\infty}\left(I d-U_{\Phi}\right)^{n}$. Consequently, for $f \in\left(\mathcal{H}_{1}^{\nu}\right)^{\urcorner}$and $\zeta \in \mathcal{H}_{1}^{\nu}$

$$
\langle f, T \zeta\rangle=\left\langle f, V^{-1} U_{\Phi}^{-1} V \zeta\right\rangle=\left\langle V f, U_{\Phi}^{-1} V \zeta\right\rangle_{L_{\infty}^{1 / \nu} \times L_{1}^{\nu}}=\left\langle U_{\Phi}^{-1} V f, V \zeta\right\rangle_{L_{\infty}^{1 / \nu} \times L_{1}^{\nu}}=\langle T f, \zeta\rangle .
$$

It follows further that $T$ is sequentially continuous with respect to the weak*-topology of $\left(\mathcal{H}_{1}^{\nu}\right)^{\urcorner}$. To see this let $f_{n} \rightarrow f$ in the weak*-topology. Then $\left\langle T f_{n}, \zeta\right\rangle=\left\langle f_{n}, T \zeta\right\rangle \rightarrow\langle f, T \zeta\rangle=\langle T f, \zeta\rangle$ for every $\zeta \in \mathcal{H}_{1}^{\nu}$. By Lemma 2.39, Corollary 2.46 and Lemma 2.32 the atoms $\varphi_{x_{i}}$ lie in $\mathcal{H}_{1}^{\nu} \cap \mathrm{Co}(Y)$. Since $T$ respects these subspaces we can define

$$
\psi_{i}:=c_{i} T \varphi_{i} \in \mathcal{H}_{1}^{\nu} \cap \operatorname{Co}(Y)
$$

and claim that $\widehat{\mathcal{F}_{d}}=\left\{\psi_{i}\right\}_{i \in I}$ is the desired "dual" of $\mathcal{F}_{d}=\left\{\varphi_{i}\right\}_{i \in I}$.

After these preliminary considerations we now turn to the proof of the assertions.

Step 1. If $f \in \operatorname{Co}(Y)$ then $\left\{\left\langle f, \varphi_{i}\right\rangle\right\}_{i \in I}=\left\{V f\left(x_{i}\right)\right\}_{i \in I} \in Y^{b}$ and $\left\|\left\{\left\langle f, \varphi_{i}\right\rangle\right\}_{i \in I}\left|Y^{b}\|\lesssim\| f\right| \operatorname{Co}(Y)\right\|$ by Corollary 2.37. Furthermore, it holds $T f \in \operatorname{Co}(Y)$ and Corollary 2.37 yields $\left\{\left\langle f, \psi_{i}\right\rangle\right\}_{i \in I}=$ $\left\{c_{i}\left\langle T f, \varphi_{i}\right\rangle\right\}_{i \in I} \in Y^{\natural}$ with the estimate $\left\|\left\{\left\langle f, \psi_{i}\right\rangle\right\}_{i \in I}\left|Y^{\natural}\|\leqslant\|\left\{\left\langle T f, \varphi_{i}\right\rangle\right\}_{i \in I}\right| Y^{b}\right\| \lesssim\|T f \mid \operatorname{Co}(Y)\| \lesssim$ $\|f \mid \mathrm{Co}(Y)\|$.

Step 2. If $\left\{\lambda_{i}\right\}_{i \in I} \in Y^{\natural}$ then by Lemma 2.40 the sum $\sum_{i \in I} \lambda_{i} \varphi_{i}$ converges in the weak*-topology to an element in $\operatorname{Co}(Y)$ with estimate $\left\|\sum_{i \in I} \lambda_{i} \varphi_{i}\left|\operatorname{Co}(Y)\|\lesssim\|\left\{\lambda_{i}\right\}_{i \in I}\right| Y^{\natural}\right\|$. If the finite sequences are dense in $Y^{b}$ (or equivalently $Y^{\natural}$ ) the convergence is even in the quasi-norm of $\mathrm{Co}(Y)$.

A similar statement holds for the dual family $\left\{\psi_{i}\right\}_{i \in I}$. Indeed, for $\left\{\lambda_{i}\right\}_{i \in I} \in Y^{b}$ we have $\left\{c_{i} \lambda_{i}\right\}_{i \in I} \in Y^{\natural}$ and hence $\sum_{i \in I} c_{i} \lambda_{i} \varphi_{i}$ converges in the weak*-topology to an element in $\operatorname{Co}(Y)$. Since $T$ is sequentially continuous it follows that

$$
\sum_{i \in I} \lambda_{i} \psi_{i}=\sum_{i \in I} c_{i} \lambda_{i} T \varphi_{i}=T\left(\sum_{i \in I} c_{i} \lambda_{i} \varphi_{i}\right) \in \operatorname{Co}(Y)
$$

with weak*-convergence in the sums. The operator $T$ is also continuous on $\mathrm{Co}(Y)$, proving the quasi-norm convergence if the finite sequences are dense. Moreover, we have the estimate

$$
\left\|\sum_{i \in I} \lambda_{i} \psi_{i}\left|\operatorname{Co}(Y)\|\lesssim\| \sum_{i \in I} c_{i} \lambda_{i} \varphi_{i}\right| \operatorname{Co}(Y)\right\| \lesssim\left\|\left\{c_{i} \lambda_{i}\right\}_{i \in I}\left|Y^{\natural}\|\leqslant\|\left\{\lambda_{i}\right\}_{i \in I}\right| Y^{\natural}\right\| .
$$

Step 3. In this step we prove the expansions in (iii). For $f \in\left(\mathcal{H}_{1}^{\nu}\right)^{\urcorner}$we have the identity

$$
V f=U_{\Phi}\left(U_{\Phi}^{-1} V f\right)=\sum_{i \in I} c_{i}\left(U_{\Phi}^{-1} V f\right)\left(x_{i}\right) R\left(\cdot, x_{i}\right)=\sum_{i \in I}\left\langle f, \psi_{i}\right\rangle V \varphi_{i}
$$

with pointwise absolute convergence a.e. in the sums. Since $\left.\left(\mathcal{H}_{1}^{\nu}\right)\right\urcorner=\operatorname{Co}\left(L_{\infty}^{1 / \nu}\right)$ the coefficients $\left\{\left\langle f, \psi_{i}\right\rangle\right\}_{i \in I}$ belong to $\left(L_{\infty}^{1 / \nu}\right)^{\natural}$ according to Step 1. Hence, by Lemma 2.40 it holds $V f=$ $V\left(\sum_{i \in I}\left\langle f, \psi_{i}\right\rangle \varphi_{i}\right)$ with weak*-convergence of the sum. The injectivity of $V$ finally yields

$$
f=\sum_{i \in I}\left\langle f, \psi_{i}\right\rangle \varphi_{i}
$$

Using the sequential continuity of $T$ with respect to the weak*-topology we can further deduce

$$
f=T T^{-1} f=\sum_{i \in I}\left\langle T^{-1} f, \psi_{i}\right\rangle T \varphi_{i}=\sum_{i \in I}\left\langle T^{-1} f, c_{i} T \varphi_{i}\right\rangle T \varphi_{i}=\sum_{i \in I}\left\langle f, \varphi_{i}\right\rangle \psi_{i} .
$$


In particular, these expansions are valid for $f \in \operatorname{Co}(Y)$ with coefficients $\left\{\left\langle f, \psi_{i}\right\rangle\right\}_{i \in I} \in Y^{\natural}$ and $\left\{\left\langle f, \varphi_{i}\right\rangle\right\}_{i \in I} \in Y^{b}$ by Step 1.

Step 4. If $f \in\left(\mathcal{H}_{1}^{\nu}\right)^{\urcorner}$and either $\left\{\left\langle f, \varphi_{i}\right\rangle\right\}_{i \in I} \in Y^{b}$ or $\left\{\left\langle f, \psi_{i}\right\rangle\right\}_{i \in I} \in Y^{\natural}$ we can conclude from the expansions (2.23) and (2.24) together with Step 2 that $f \in \operatorname{Co}(Y)$. Moreover, $\left\|\left\{\left\langle f, \psi_{i}\right\rangle\right\}_{i \in I} \mid Y^{\natural}\right\|$ and $\left\|\left\{\left\langle f, \varphi_{i}\right\rangle\right\}_{i \in I} \mid Y^{b}\right\|$ are equivalent quasi-norms on $\operatorname{Co}(Y)$ because using Steps 1 and 2

and

$$
\begin{gathered}
\left\|f\left|\operatorname{Co}(Y)\|=\| \sum_{i \in I}\left\langle f, \psi_{i}\right\rangle \varphi_{i}\right| \operatorname{Co}(Y)\right\| \lesssim\left\|\left\{\left\langle f, \psi_{i}\right\rangle\right\}_{i \in I}\left|Y^{\natural}\|\lesssim\| f\right| \operatorname{Co}(Y)\right\| \\
\left\|f\left|\operatorname{Co}(Y)\|=\| \sum_{i \in I}\left\langle f, \varphi_{i}\right\rangle \psi_{i}\right| \operatorname{Co}(Y)\right\| \lesssim\left\|\left\{\left\langle f, \varphi_{i}\right\rangle\right\}_{i \in I}\left|Y^{b}\|\lesssim\| f\right| \operatorname{Co}(Y)\right\| .
\end{gathered}
$$

Remark 2.49. Properties (i)-(iii) in particular show that the discrete families $\mathcal{F}_{d}$ and $\widehat{\mathcal{F}}_{d}$ both constitute atomic decompositions for $\mathrm{Co}(Y)$, as well as quasi-Banach frames, compare e.g. [50, 48].

\section{Frame expansion}

Now we come to another main discretization result, which allows to discretize the coorbit space $\mathrm{Co}(Y)=\operatorname{Co}(\mathcal{F}, Y)$ by samples of a frame $\mathcal{G}=\left\{\psi_{x}\right\}_{x \in X}$ different from the analyzing frame $\mathcal{F}$. It is a generalization of [50, Thm. 3.14], whose original proof carries over to the quasi-Banach setting based on Corollary 2.37 and Lemma 2.40. In contrast to Theorem 2.48, here we require the additional property of the covering $\mathcal{U}=\left\{U_{i}\right\}_{i \in I}$ that for some constant $D>0$

$$
\mu\left(U_{i}\right) \geqslant D \text { for all } i \in I \text {. }
$$

Theorem 2.50. Let $Y$ be a rich solid QBF-space on $X$ and assume that the analyzing frame $\mathcal{F}=\left\{\varphi_{x}\right\}_{x \in X}$ has property $F(\nu, Y)$. For $r \in\{1, \ldots, n\}$ let $\mathcal{G}_{r}=\left\{\psi_{x}^{r}\right\}_{x \in X}$ and $\tilde{\mathcal{G}}_{r}=\left\{\tilde{\psi}_{x}^{r}\right\}_{x \in X}$ be families in $\mathcal{H}$, and suppose that for some admissible covering $\mathcal{U}=\left\{U_{i}\right\}_{i \in I}$ with the additional property 2.25) the kernels $K_{r}:=K_{\mathcal{U}}\left[\mathcal{G}_{r}, \mathcal{F}\right]$ and $\tilde{K}_{r}^{*}:=K_{\mathcal{U}}^{*}\left[\tilde{\mathcal{G}}_{r}, \mathcal{F}\right]$ belong to $\mathcal{B}_{Y, m_{\nu}}$. Then, if every $f \in \mathcal{H}$ has an expansion

$$
f=\sum_{r=1}^{n} \sum_{i \in I}\left\langle f, \tilde{\psi}_{x_{i}}^{r}\right\rangle \psi_{x_{i}}^{r}
$$

with fixed points $x_{i} \in U_{i}$, this expansion extends to all $f \in \operatorname{Co}(Y)=\operatorname{Co}(\mathcal{F}, Y)$. Furthermore, $\left.f \in\left(H_{\nu}^{1}\right)\right\urcorner$ belongs to $\mathrm{Co}(Y)$ if and only if $\left\{\left\langle f, \tilde{\psi}_{x_{i}}^{r}\right\rangle\right\}_{i \in I} \in Y^{\natural}(\mathcal{U})$ for each $r \in\{1, \ldots, n\}$, and in this case we have $\left\|f\left|\mathrm{Co}(Y)\left\|=\sum_{r=1}^{n}\right\|\left\{\left\langle f, \tilde{\psi}_{x_{i}}^{r}\right\rangle\right\}_{i \in I}\right| Y^{\natural}(\mathcal{U})\right\|$. The convergence in (2.26) is in the quasi-norm of $\mathrm{Co}(Y)$ if the finite sequences are dense in $Y^{\natural}(\mathcal{U})$. In general, we have weak*-convergence induced by $\left(\mathcal{H}_{1}^{\nu}\right)^{\urcorner}$.

Observe that the technical assumption $Y^{\natural} \hookrightarrow\left(L_{\infty}^{1 / v}\right)^{\natural}$ made in [50, Thm. 3.14] is not necessary. In view of Lemma 2.13 it is further not necessary to require $\mathcal{G}_{r}, \tilde{\mathcal{G}}_{r} \subset \mathcal{H}_{1}^{\nu}$. In fact, $K_{r}, \tilde{K}_{r}^{*} \in \mathcal{A}_{m_{\nu}}$ is a stronger condition than $G\left[\mathcal{G}_{r}, \mathcal{F}\right], G^{*}\left[\tilde{\mathcal{G}}_{r}, \mathcal{F}\right] \in \mathcal{A}_{m_{\nu}}$ and implies $\mathcal{G}_{r}, \tilde{\mathcal{G}}_{r} \subset \mathcal{H}_{1}^{\nu}$.

\section{$3 \quad$ Variable exponent spaces}

In the remainder we give a demonstration of the theory. As an example we will show that variable exponent spaces, which have caught some attention recently, fall into the framework of coorbit theory and can be handled conveniently within the theory. 


\subsection{Spaces of variable integrability}

The spaces of variable integrability $L_{p(\cdot)}\left(\mathbb{R}^{d}\right)$ were first introduced by Orlicz [46] in 1931 as a generalization of the Lebesgue spaces $L_{p}\left(\mathbb{R}^{d}\right)$. Before defining them let us introduce some standard notation from [41]. For a measurable function $p: \mathbb{R}^{d} \rightarrow(0, \infty]$ and a set $\Omega \subset \mathbb{R}^{d}$ we define the quantities $p_{\Omega}^{-}=\underset{x \in \Omega}{\operatorname{essinf}} p(x)$ and $p_{\Omega}^{+}=\underset{x \in \Omega}{\operatorname{ess} \sup } p(x)$. Furthermore, we abbreviate $p^{-}=p_{\mathbb{R}^{d}}^{-}$and $p^{+}=p_{\mathbb{R}^{d}}^{+}$and say that $p(\cdot)$ belongs to the class of admissible exponents $\mathcal{P}\left(\mathbb{R}^{d}\right)$ if $p^{-}>0$. Having an admissible exponent $p \in \mathcal{P}\left(\mathbb{R}^{d}\right)$ we define the set $\mathbb{R}_{\infty}^{d}=\left\{x \in \mathbb{R}^{d}: p(x)=\infty\right\}$ and for every measurable function $f: \mathbb{R}^{d} \rightarrow \mathbb{C}$ the modular

$$
\varrho_{p(\cdot)}(f)=\int_{\mathbb{R}^{d} \backslash \mathbb{R}_{\infty}^{d}}|f(x)|^{p(x)} d x+\underset{x \in \mathbb{R}_{\infty}^{d}}{\operatorname{ess} \sup }|f(x)| .
$$

Definition 3.1. The space $L_{p(\cdot)}\left(\mathbb{R}^{d}\right)$ is the collection of all functions $f$ such that there exists a $\lambda>0$ with $\varrho_{p(\cdot)}(\lambda f)<\infty$. It is equipped with the Luxemburg quasi-norm

$$
\left\|f \mid L_{p(\cdot)}\left(\mathbb{R}^{d}\right)\right\|=\inf \left\{\lambda>0: \varrho_{p(\cdot)}\left(\frac{f}{\lambda}\right)<1\right\} .
$$

The spaces $L_{p(\cdot)}\left(\mathbb{R}^{d}\right)$ share many properties with the constant exponent spaces $L_{p}\left(\mathbb{R}^{d}\right)$. Let us mention a few; the proofs can be found in [41] and in [16]:

- If $p(x)=p$ then $L_{p(\cdot)}\left(\mathbb{R}^{d}\right)=L_{p}\left(\mathbb{R}^{d}\right)$,

- if $|f(x)| \geqslant|g(x)|$ for a.e. $x \in \mathbb{R}^{d}$ then $\varrho_{p(\cdot)}(f) \geqslant \varrho_{p(\cdot)}(g)$ and $\left\|f\left|L_{p(\cdot)}\left(\mathbb{R}^{d}\right)\|\geqslant\| g\right| L_{p(\cdot)}\left(\mathbb{R}^{d}\right)\right\|$,

- $\varrho_{p(\cdot)}(f)=0$ if and only if $f=0$,

- for $p(\cdot) \geqslant 1$ Hölder's inequality holds [41, Theorem 2.1]

$$
\int_{\mathbb{R}^{d}}|f(x) g(x)| d x \leqslant 4\left\|f\left|L_{p(\cdot)}\left(\mathbb{R}^{d}\right)\|\| g\right| L_{p^{\prime}(\cdot)}\left(\mathbb{R}^{d}\right)\right\|
$$

where $1 / p(\cdot)+1 / p^{\prime}(\cdot)=1$ pointwise.

There are also some properties of the usual constant exponent spaces which the $L_{p(\cdot)}\left(\mathbb{R}^{d}\right)$ spaces do not share. For example in general the $L_{p(\cdot)}\left(\mathbb{R}^{d}\right)$ spaces are not translation invariant, i.e. $f \in L_{p(\cdot)}\left(\mathbb{R}^{d}\right)$ does not automatically imply that $f(\cdot+h)$ belongs to $L_{p(\cdot)}\left(\mathbb{R}^{d}\right)$ for $h \in \mathbb{R}^{d}$. As a consequence also Young's convolution inequality is not valid (see again [41] for details).

The breakthrough for $L_{p(\cdot)}\left(\mathbb{R}^{d}\right)$ spaces was made by Diening in [14] when he showed that the Hardy-Littlewood maximal operator $\mathcal{M}$ is bounded on $L_{p(\cdot)}\left(\mathbb{R}^{d}\right)$ under certain regularity conditions on $p(\cdot)$. His result has been generalized in many cases (see [15, [45] and [7]) and it turned out that logarithmic Hölder continuity classes are well adapted to the boundedness of the maximal operator.

Definition 3.2. Let $g \in C\left(\mathbb{R}^{d}\right)$. We say that $g$ is locally log-Hölder continuous, abbreviated $g \in C_{\mathrm{loc}}^{\log }\left(\mathbb{R}^{d}\right)$, if there exists $c_{\log }>0$ such that

$$
|g(x)-g(y)| \leqslant \frac{c_{\log }}{\log (e+1 /|x-y|)} \quad \text { for all } x, y \in \mathbb{R}^{d}
$$


We say that $g$ is globally log-Hölder continuous, abbreviated $g \in C^{\log }\left(\mathbb{R}^{d}\right)$, if $g$ is locally $\log$ Hölder continuous and there exists $g_{\infty} \in \mathbb{R}$ such that

$$
\left|g(x)-g_{\infty}\right| \leqslant \frac{c_{\log }}{\log (e+|x|)} \quad \text { for all } x \in \mathbb{R}^{d} .
$$

With the help of the above logarithmic Hölder continuity the following result holds.

Lemma 3.3 ([15, Thm. 3.6]). Let $p \in \mathcal{P}\left(\mathbb{R}^{d}\right)$ with $1<p^{-} \leqslant p^{+} \leqslant \infty$. If $\frac{1}{p} \in C^{\log }\left(\mathbb{R}^{d}\right)$, then $\mathcal{M}$ is bounded on $L_{p(\cdot)}\left(\mathbb{R}^{d}\right)$ i.e., there exists $c>0$ such that for all $f \in L_{p(\cdot)}\left(\mathbb{R}^{d}\right)$

$$
\left\|\mathcal{M} f\left|L_{p(\cdot)}\left(\mathbb{R}^{d}\right)\|\leqslant c\| f\right| L_{p(\cdot)}\left(\mathbb{R}^{d}\right)\right\|
$$

Since logarithmic Hölder continuous exponents play an essential role we introduce the class $\mathcal{P}^{\log }\left(\mathbb{R}^{d}\right)$ of admissible exponents $p(\cdot)$ with $1 / p \in C^{\log }\left(\mathbb{R}^{d}\right)$ and $0<p^{-} \leqslant p^{+} \leqslant \infty$. As a consequence of Lemma 3.3. for exponents $p \in \mathcal{P}^{\log }\left(\mathbb{R}^{d}\right)$ the maximal operator $\mathcal{M}$ is bounded on $L_{\frac{p(\cdot)}{t}}\left(\mathbb{R}^{d}\right)$ for every $0<t<p^{-}$.

\section{$3.2 \quad 2$-microlocal function spaces with variable integrability}

We proceed with spaces of Besov-Triebel-Lizorkin type featuring variable integrability and

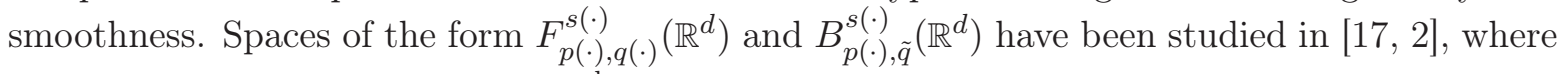
$s: \mathbb{R}^{d} \rightarrow \mathbb{R}$ with $s \in L_{\infty}\left(\mathbb{R}^{d}\right) \cap C_{\mathrm{loc}}^{\log }\left(\mathbb{R}^{d}\right)$. A further generalization was pursued in [36, 37] replacing the smoothness parameter $s(\cdot)$ by a more general weight function $w$. We make some reasonable restrictions on $w$ and use the class $\mathcal{W}_{\alpha_{1}, \alpha_{2}}^{\alpha_{3}}$ of admissible weights introduced in [36].

Definition 3.4. For real numbers $\alpha_{3} \geqslant 0$ and $\alpha_{1} \leqslant \alpha_{2}$ a weight function $w: X \rightarrow(0, \infty)$ on the index set $X=\mathbb{R}^{d} \times[(0,1) \cup\{\infty\}]$ belongs to the class $\mathcal{W}_{\alpha_{1}, \alpha_{2}}^{\alpha_{3}}$ if and only if for $\mathbf{x}=(x, t) \in X$,

$$
\begin{aligned}
& \text { (W1) }\left\{\begin{array}{l}
\left(\frac{s}{t}\right)^{\alpha_{1}} w(x, s) \leqslant w(x, t) \leqslant\left(\frac{s}{t}\right)^{\alpha_{2}} w(x, s) \quad, \quad s \geqslant t \\
t^{-\alpha_{1}} w(x, \infty) \leqslant w(x, t) \leqslant t^{-\alpha_{2}} w(x, \infty) \quad, \quad s=\infty,
\end{array}\right. \\
& \text { (W2) } w(x, t) \leqslant w(y, t)\left\{\begin{array}{cc}
(1+|x-y| / t)^{\alpha_{3}}, & t \in(0,1) \\
(1+|x-y|)^{\alpha_{3}}, & , t=\infty
\end{array} \text { for all } y \in \mathbb{R}^{d} .\right.
\end{aligned}
$$

Example 3.5. The main examples are weights of the form

$$
w_{s, s^{\prime}}(x, t)=\left\{\begin{array}{cl}
t^{-s\left(1+\frac{\left|x-x_{0}\right|}{t}\right)^{s^{\prime}}}, \quad t \in(0,1) \\
\left(1+\left|x-x_{0}\right|\right)^{s^{\prime}}, & t=\infty
\end{array} .\right.
$$

where $s, s^{\prime} \in \mathbb{R}$. These weights are continuous versions of 2-microlocal weights, used to define 2-microlocal function spaces of Besov-Lizorkin-Triebel type, see [36, 38, 37].

By choosing $s^{\prime}=0$ we get back to usual Besov-Lizorkin-Triebel spaces with smoothness $s \in \mathbb{R}$.

The special weights from this example are usually called 2-microlocal weights. Furthermore, function spaces which are defined with admissible weights $w \in \mathcal{W}_{\alpha_{1}, \alpha_{2}}^{\alpha_{3}}$ are usually called 2microlocal spaces. This term was coined by Bony [4] and Jaffard [35], who also introduced the concept of 2-microlocal analysis to study local regularity of functions. 
Remark 3.6. By the conditions on admissible weights $w \in \mathcal{W}_{\alpha_{1}, \alpha_{2}}^{\alpha_{3}}$ we obtain the following estimates which will be useful later on:

1. For $s \leqslant t$ we get from (W1)

$$
\left(\frac{s}{t}\right)^{\alpha_{2}} w(x, s) \leqslant w(x, t) \leqslant\left(\frac{s}{t}\right)^{\alpha_{1}} w(x, s) .
$$

2. For $0<c<s / t$ we have from (W1) and (3.1)

$$
\frac{w(x, t)}{w(x, s)} \leqslant \max \left\{1, c^{\alpha_{1}-\alpha_{2}}\right\}\left(\frac{s}{t}\right)^{\alpha_{2}} .
$$

3. For $0<c<t / s$ we obtain similarly from (W1) and (3.1)

$$
\frac{w(x, t)}{w(x, s)} \leqslant \max \left\{1, c^{\alpha_{1}-\alpha_{2}}\right\}\left(\frac{s}{t}\right)^{\alpha_{1}} \text {. }
$$

4. Consequently, we have for $0<c_{1}<s / t<c_{2}$ from (3.2) and (3.3)

$$
w(x, t)=w(x, s) \quad \text { for all } x \in \mathbb{R}^{d} .
$$

5. Using (W2) and the inequalities (3.2) and (3.3) we can relate $w(x, t)$ to $w(0,1 / 2)$ by

$$
w(0,1 / 2) t^{-\alpha_{1}}(1+|x|)^{-\alpha_{3}} \lesssim w(x, t) \lesssim w(0,1 / 2) t^{-\alpha_{2}}(1+|x|)^{\alpha_{3}} .
$$

A weight $w \in \mathcal{W}_{\alpha_{1}, \alpha_{2}}^{\alpha_{3}}$ gives rise to a semi-discrete counterpart $\left(w_{j}\right)_{j \in \mathbb{N}_{0}}$, corresponding to an admissible weight sequence in the sense of [36, 38, 37, given by

$$
w_{j}(x)=\left\{\begin{array}{rll}
w\left(x, 2^{-j}\right) & , j \in \mathbb{N} \\
w(x, \infty) & , \quad j=0
\end{array}\right.
$$

In [37, Lemma 2.6] it was shown that it is equivalent to consider a smoothness function $s \in L_{\infty}\left(\mathbb{R}^{d}\right) \cap C_{\operatorname{loc}}^{\log }\left(\mathbb{R}^{d}\right)$ or an admissible weight sequence stemming from $w \in \mathcal{W}_{\alpha_{1}, \alpha_{2}}^{\alpha_{3}}$ if they are connected by $w_{j}(x)=2^{j s(x)}$, see (3.4). But there exist weight sequences (Example 3.5 with $s^{\prime} \neq 0$ ) where it is not possible to find a smoothness function $s: \mathbb{R}^{d} \rightarrow \mathbb{R}$ such that the above relation holds.

Recently in [58] the concept of admissible weight sequences was extended to include more general weights. We will not follow this generalization of admissible weights, but we remark that by this definition we can have local Muckenhoupt weights as components in the sequence.

The spaces $B_{p(\cdot), \tilde{q}}^{w}\left(\mathbb{R}^{d}\right)$ and $F_{p(\cdot), q(\cdot)}^{w}\left(\mathbb{R}^{d}\right)$ are defined Fourier analytical as subspaces of the tempered distributions $\mathcal{S}^{\prime}\left(\mathbb{R}^{d}\right)$. As usual the Schwartz space $\mathcal{S}\left(\mathbb{R}^{d}\right)$ denotes the locally convex space of rapidly decreasing infinitely differentiable functions on $\mathbb{R}^{d}$. Its topology is generated by the seminorms

$$
\|\varphi\|_{k, l}=\sup _{x \in \mathbb{R}^{d}}(1+|x|)^{k} \sum_{|\beta| \leqslant l}\left|D^{\beta} \varphi(x)\right|
$$

for every $k, l \in \mathbb{N}_{0}$. Its topological dual, the space of tempered distributions on $\mathbb{R}^{d}$, is denoted by $\mathcal{S}^{\prime}\left(\mathbb{R}^{d}\right)$. The Fourier transform and its inverse are defined on both $\mathcal{S}\left(\mathbb{R}^{d}\right)$ and $\mathcal{S}^{\prime}\left(\mathbb{R}^{d}\right.$ ) (see 
Appendix A.1) and we denote them by $\hat{f}$ and $f^{\vee}$. Finally, we introduce the subspace $\mathcal{S}_{0}\left(\mathbb{R}^{d}\right)$ of $\mathcal{S}\left(\mathbb{R}^{d}\right)$ by

$$
\mathcal{S}_{0}\left(\mathbb{R}^{d}\right):=\left\{f \in \mathcal{S}\left(\mathbb{R}^{d}\right): D^{\bar{\alpha}} \widehat{f}(0)=0 \text { for every multi-index } \bar{\alpha} \in \mathbb{N}_{0}^{d}\right\} .
$$

The definition of $B_{p(\cdot), \tilde{q}^{w}}^{w}\left(\mathbb{R}^{d}\right)$ and $F_{p(\cdot), q(\cdot)}^{w}\left(\mathbb{R}^{d}\right)$ relies on a dyadic decomposition of unity, see also [55, 2.3.1].

Definition 3.7. Let $\Pi\left(\mathbb{R}^{d}\right)$ be the collection of all systems $\left\{\varphi_{j}\right\}_{j \in \mathbb{N}_{0}} \subset \mathcal{S}\left(\mathbb{R}^{d}\right)$ such that

(i) there is a function $\varphi \in \mathcal{S}\left(\mathbb{R}^{d}\right)$ with $\varphi_{j}(\xi)=\varphi\left(2^{-j} \xi\right), j \in \mathbb{N}$,

(ii) $\operatorname{supp} \varphi_{0} \subset\left\{\xi \in \mathbb{R}^{d}:|\xi| \leqslant 2\right\}, \quad \operatorname{supp} \varphi \subset\left\{\xi \in \mathbb{R}^{d}: 1 / 2 \leqslant|\xi| \leqslant 2\right\}$,

(iii) $\sum_{j=0}^{\infty} \varphi_{j}(\xi)=1$ for every $\xi \in \mathbb{R}^{d}$.

Definition 3.8. Let $\left\{\varphi_{j}\right\}_{j=0}^{\infty} \in \Pi\left(\mathbb{R}^{d}\right)$ and put $\widehat{\Phi}_{j}=\varphi_{j}$ for $j \in \mathbb{N}_{0}$. Let further $w \in \mathcal{W}_{\alpha_{1}, \alpha_{2}}^{\alpha_{3}}$ with associated weight sequence $\left\{w_{j}\right\}_{j \in \mathbb{N}_{0}}$ defined as in (3.4).

(i) For $p \in \mathcal{P}\left(\mathbb{R}^{d}\right), \tilde{q} \in(0, \infty]$, we define $B_{p(\cdot), \tilde{q}}^{w}\left(\mathbb{R}^{d}\right)=\left\{f \in \mathcal{S}^{\prime}\left(\mathbb{R}^{d}\right):\left\|f \mid B_{p(\cdot), \tilde{q}}^{w}\left(\mathbb{R}^{d}\right)\right\|<\infty\right\}$ with

$$
\left\|f \mid B_{p(\cdot), \tilde{q}}^{w}\left(\mathbb{R}^{d}\right)\right\|=\left(\sum_{j=0}^{\infty}\left\|w_{j}(\cdot)\left(\Phi_{j} * f\right)(\cdot) \mid L_{p(\cdot)}\left(\mathbb{R}^{d}\right)\right\|^{\tilde{q}}\right)^{1 / \tilde{q}} .
$$

(ii) For $p, q \in \mathcal{P}\left(\mathbb{R}^{d}\right)$ we define $F_{p(\cdot), q(\cdot)}^{w}\left(\mathbb{R}^{d}\right)=\left\{f \in \mathcal{S}^{\prime}\left(\mathbb{R}^{d}\right):\left\|f \mid F_{p(\cdot), q(\cdot)}^{w}\left(\mathbb{R}^{d}\right)\right\|<\infty\right\}$ with

$$
\left\|f\left|F_{p(\cdot), q(\cdot)}^{w}\left(\mathbb{R}^{d}\right)\|=\|\left(\sum_{j=0}^{\infty}\left|w_{j}(\cdot)\left(\Phi_{j} * f\right)(\cdot)\right|^{q(\cdot)}\right)^{1 / q(\cdot)}\right| L_{p(\cdot)}\left(\mathbb{R}^{d}\right)\right\| .
$$

Remark 3.9. It is also possible to consider Besov spaces $B_{p(\cdot), q(\cdot)}^{w}\left(\mathbb{R}^{d}\right)$ with variable index $q(\cdot)$, which were introduced and studied in [2]. The definition of these spaces is very technical since they require a new modular. Surprisingly it is much harder to work with Besov spaces with variable indices $p(\cdot)$ and $q(\cdot)$ than to work with variable Triebel-Lizorkin spaces, in sharp contrast to the constant exponent case. For example, Besov spaces with variable $q(\cdot)$ are not always normed spaces for $\min \{p(\cdot), q(\cdot)\} \geqslant 1$, even if $p(\cdot)$ is a constant (see [40] for details). So we restrict our studies on Besov spaces to the case were the index $q(\cdot)$ remains a constant $\tilde{q}$ and we leave the fully variable case for further research.

Formally, the definition of $F_{p(\cdot), q(\cdot)}^{w}\left(\mathbb{R}^{d}\right)$ and $B_{p(\cdot), \tilde{q}}^{w}\left(\mathbb{R}^{d}\right)$ depends on the chosen decomposition of unity $\left\{\varphi_{j}\right\}_{j=0}^{\infty} \in \Pi\left(\mathbb{R}^{d}\right)$. The following characterization by local means shows that under certain regularity conditions on the indices $p(\cdot), q(\cdot)$ it is in fact independent, in the sense of equivalent quasi-norms.

To get useful further characterizations of the spaces defined above we need a replacement for the classical Fefferman-Stein maximal inequality since it does not hold in our case if $q(\cdot)$ is non-constant. We will use the following convolution inequality. 
Lemma 3.10 (Theorem 3.2 in [17]). Let $p, q \in \mathcal{P}^{\log }\left(\mathbb{R}^{d}\right)$ with $1<p^{-} \leqslant p^{+}<\infty$ and $1<q^{-} \leqslant$ $q^{+}<\infty$, then for $m>d$ there exists a constant $c>0$ such that

$$
\|\|\left(\eta_{\nu, m} * f_{\nu}\right)_{\nu \in \mathbb{N}_{0}}\left|\ell _ { q ( \cdot ) } \left\|\left|L _ { p ( \cdot ) } ( \mathbb { R } ^ { d } ) \| \leqslant c \| \left\|( f _ { \nu } ) _ { \nu \in \mathbb { N } _ { 0 } } \left|\ell_{q(\cdot)}\left\|\mid L_{p(\cdot)}\left(\mathbb{R}^{d}\right)\right\|,\right.\right.\right.\right.\right.
$$

where $\eta_{\nu, m}(x)=2^{\nu d}\left(1+2^{\nu}|x|\right)^{-m}$.

\subsection{Continuous local means characterization}

For our purpose, it is more convenient to reformulate Definition 3.8 in terms of a continuous characterization, where the discrete dilation parameter $j \in \mathbb{N}_{0}$ is replaced by $t>0$ and the sums become integrals over $t$. Characterizations of this type have some history and are usually referred to as characterizations via (continuous) local means. For further references and some historical facts we mainly refer to [57, 5, 52] and in particular to the recent contribution [59], which provides a complete and self-contained reference.

The system $\left\{\varphi_{j}\right\}_{j \in \mathbb{N}_{0}} \in \Pi\left(\mathbb{R}^{d}\right)$ may be replaced by a more general one. Essential are functions $\Phi_{0}, \Phi \in \mathcal{S}\left(\mathbb{R}^{d}\right)$ satisfying the so-called Tauberian conditions

$$
\begin{array}{rll}
\left|\widehat{\Phi}_{0}(\xi)\right|>0 & \text { on } & \{|\xi|<2 \varepsilon\}, \\
|\widehat{\Phi}(\xi)|>0 & \text { on } & \{\varepsilon / 2<|\xi|<2 \varepsilon\}
\end{array}
$$

for some $\varepsilon>0$, and - for some $R+1 \in \mathbb{N}_{0}$ - the moment conditions

$$
D^{\beta} \widehat{\Phi}(0)=0 \text { for all }|\beta|_{1} \leqslant R .
$$

If $R+1=0$ the condition (3.6) is void. We will call the functions $\Phi_{0}$ and $\Phi$ kernels for local means and use the notations $\Phi_{k}=2^{k d} \Phi\left(2^{k} \cdot\right), k \in \mathbb{N}$, as well as $\Phi_{t}=\mathcal{D}_{t} \Phi=t^{-d} \Phi(\cdot / t)$ for $t>0$. The associated Peetre maximal function

$$
\left(\Phi_{t}^{*} f\right)_{a}(x)=\sup _{y \in \mathbb{R}^{d}} \frac{\left|\left(\Phi_{t} * f\right)(x+y)\right|}{(1+|y| / t)^{a}} \quad, \quad x \in \mathbb{R}^{d}, t>0,
$$

was introduced in [47] for $f \in \mathcal{S}^{\prime}\left(\mathbb{R}^{d}\right)$ and $a>0$. We also need the stronger version

$$
\left\langle\Phi_{t}^{*} f\right\rangle_{a}(x)=\sup _{\substack{t \\ \frac{t}{\tau<\tau \leqslant 2 t} \\ \tau<1}}\left(\Phi_{\tau}^{*} f\right)_{a}(x) \quad, \quad x \in \mathbb{R}^{d}, t>0, \quad(\text { Convention: } \sup \varnothing=0)
$$

which we will refer to as Peetre-Wiener maximal function and which was utilized for the coorbit characterization of the classical Besov-Lizorkin-Triebel-spaces in [53]. To adapt to the inhomogeneous setting we further put $\left\langle\Phi_{0}^{*} f\right\rangle_{a}=\left(\Phi_{0}^{*} f\right)_{a}=\left(\left(\Phi_{0}\right)_{1}^{*} f\right)_{a}$.

Using these maximal functions we now state several different characterizations.

Theorem 3.11. Let $w \in \mathcal{W}_{\alpha_{1}, \alpha_{2}}^{\alpha_{3}}$ and choose functions $\Phi_{0}, \Phi \in \mathcal{S}\left(\mathbb{R}^{d}\right)$ satisfying (3.5) and (3.6) with $R+1>\alpha_{2}$. For $x \in \mathbb{R}^{d}$ and $t \in(0,1)$ define $A_{1} f(x, t):=\left(\Phi_{t} * f\right)(x), A_{2} f(x, t):=$ $\left(\Phi_{t}^{*} f\right)_{a}(x)$, and $A_{3} f(x, t):=\left\langle\Phi_{t}^{*} f\right\rangle_{a}(x), a>0$. Further, put $A_{1} f(x, \infty):=\left(\Phi_{0} * f\right)(x)$, $A_{2} f(x, \infty):=\left(\Phi_{0}^{*} f\right)_{a}(x)$, and $A_{3} f(x, \infty):=\left\langle\Phi_{0}^{*} f\right\rangle_{a}(x)$. 
(i) If $p \in \mathcal{P}^{\log }\left(\mathbb{R}^{d}\right), 0<\tilde{q} \leqslant \infty$, and $a>\frac{d}{p^{-}}+\alpha_{3}$ then

$$
B_{p(\cdot), \tilde{q}}^{w}\left(\mathbb{R}^{d}\right)=\left\{f \in \mathcal{S}^{\prime}\left(\mathbb{R}^{d}\right):\left\|f \mid B_{p(\cdot), \tilde{q}}^{w}\left(\mathbb{R}^{d}\right)\right\|_{i}<\infty\right\} \quad, \quad i=1,2,3,4,
$$

where for $i=1,2,3$

$$
\begin{aligned}
\left\|f \mid B_{p(\cdot), \tilde{q}}^{w}\left(\mathbb{R}^{d}\right)\right\|_{i} & =\left\|w(\cdot, \infty) A_{i} f(\cdot, \infty) \mid L_{p(\cdot)}\left(\mathbb{R}^{d}\right)\right\| \\
& +\left(\int_{0}^{1}\left\|w(\cdot, t) A_{i} f(\cdot, t) \mid L_{p(\cdot)}\left(\mathbb{R}^{d}\right)\right\|^{\tilde{q}} \frac{d t}{t}\right)^{1 / \tilde{q}}, \\
\left\|f \mid B_{p(\cdot), \tilde{q}}^{w}\left(\mathbb{R}^{d}\right)\right\|_{4} & =\left\|w(\cdot, \infty)\left(\Phi_{0}^{*} f\right)_{a}(\cdot) \mid L_{p(\cdot)}\left(\mathbb{R}^{d}\right)\right\| \\
& +\left(\sum_{j=1}^{\infty}\left\|w_{j}(\cdot)\left(\Phi_{2^{-j}}^{*} f\right)_{a}(\cdot) \mid L_{p(\cdot)}\left(\mathbb{R}^{d}\right)\right\|^{\tilde{q}}\right)^{1 / \tilde{q}} .
\end{aligned}
$$

and

Moreover, $\left\|\cdot \mid B_{p(\cdot), \tilde{q}}^{w}\left(\mathbb{R}^{d}\right)\right\|_{i}, i=1,2,3,4$, are equivalent quasi-norms in $B_{p(\cdot), \tilde{q}^{w}}^{w}\left(\mathbb{R}^{d}\right)$.

(ii) If $p, q \in \mathcal{P}^{\log }\left(\mathbb{R}^{d}\right)$ with $0<q^{-} \leqslant q^{+}<\infty, 0<p^{-} \leqslant p^{+}<\infty$, and $a>\max \left\{\frac{d}{p^{-}}, \frac{d}{q^{-}}\right\}+\alpha_{3}$ then

$$
F_{p(\cdot), q(\cdot)}^{w}\left(\mathbb{R}^{d}\right)=\left\{f \in \mathcal{S}^{\prime}\left(\mathbb{R}^{d}\right):\left\|f \mid F_{p(\cdot), q(\cdot)}^{w}\left(\mathbb{R}^{d}\right)\right\|_{i}<\infty\right\} \quad, \quad i=1,2,3,4,
$$

where for $i=1,2,3$

$$
\begin{aligned}
\left\|f \mid F_{p(\cdot), q(\cdot)}^{w}\left(\mathbb{R}^{d}\right)\right\|_{i} & =\left\|w(\cdot, \infty) A_{i} f(\cdot, \infty) \mid L_{p(\cdot)}\left(\mathbb{R}^{d}\right)\right\| \\
& +\left\|\left(\int_{0}^{1}\left|w(\cdot, t) A_{i} f(\cdot, t)\right|^{q(\cdot)} \frac{d t}{t}\right)^{1 / q(\cdot)} \mid L_{p(\cdot)}\left(\mathbb{R}^{d}\right)\right\|,
\end{aligned}
$$

and

$$
\begin{aligned}
\left\|f \mid F_{p(\cdot), q(\cdot)}^{w}\left(\mathbb{R}^{d}\right)\right\|_{4} & =\left\|w(\cdot, \infty)\left(\Phi_{0}^{*} f\right)_{a}(\cdot) \mid L_{p(\cdot)}\left(\mathbb{R}^{d}\right)\right\| \\
& +\left\|\left(\sum_{j=1}^{\infty}\left|w_{j}(\cdot)\left(\Phi_{2^{-j}}^{*} f\right)_{a}(\cdot)\right|^{q(\cdot)}\right)^{1 / q(\cdot)} \mid L_{p(\cdot)}\left(\mathbb{R}^{d}\right)\right\| .
\end{aligned}
$$

Moreover, $\left\|\cdot \mid F_{p(\cdot), q(\cdot)}^{w}\left(\mathbb{R}^{d}\right)\right\|_{i}, i=1,2,3,4$, are equivalent quasi-norms in $F_{p(\cdot), q(\cdot)}^{w}\left(\mathbb{R}^{d}\right)$.

Before we present a sketch of the proof recall an important convolution inequality from [36].

Lemma 3.12. Let $0<\tilde{q} \leqslant \infty, \delta>0$ and $p, q \in \mathcal{P}\left(\mathbb{R}^{d}\right)$. Let $\left(g_{k}\right)_{k \in \mathbb{N}_{0}}$ be a sequence of nonnegative measurable functions on $\mathbb{R}^{d}$ and denote $G_{\ell}=\sum_{k=0}^{\infty} 2^{-|\ell-k| \delta} g_{k}$ for $\ell \in \mathbb{N}_{0}$. Then there exist constants $C_{1}, C_{2} \geqslant 0$ such that

$\left\|\left\{G_{\ell}\right\}_{\ell}\left|\ell_{\tilde{q}}\left(L_{p(\cdot)}\right)\left\|\leqslant C_{1}\right\|\left\{g_{k}\right\}_{k}\right| \ell_{\tilde{q}}\left(L_{p(\cdot)}\right)\right\| \quad$ and $\quad\left\|\left\{G_{\ell}\right\}_{\ell}\left|L_{p(\cdot)}\left(\ell_{q(\cdot)}\right)\left\|\leqslant C_{2}\right\|\left\{g_{k}\right\}_{k}\right| L_{p(\cdot)}\left(\ell_{q(\cdot)}\right)\right\|$.

Proof of Theorem 3.11. We only prove (ii) and comment afterwards briefly on the necessary modifications for (i). The arguments are more or less the same as in the proofs of [59, Thm. 2.6] and [53, Thm. 9.6]. We remark that the equivalences $\left\|\cdot\left|F_{p(\cdot), q(\cdot)}^{w}\left(\mathbb{R}^{d}\right)\|=\| \cdot\right| F_{p(\cdot), q(\cdot)}^{w}\left(\mathbb{R}^{d}\right)\right\|_{4}$ and $\left\|\cdot\left|B_{p(\cdot), \tilde{q}}^{w}\left(\mathbb{R}^{d}\right)\|=\| \cdot\right| B_{p(\cdot), \tilde{q}}^{w}\left(\mathbb{R}^{d}\right)\right\|_{4}$ are already known, see [36].

Step 1. First, we prove a central estimate (3.9) between different start functions $\Phi$ and $\Psi$ incorporating the different types of Peetre maximal operators. The needed norm inequalities in the theorem are consequences of this central estimate (3.9), and are subsequently deduced in the following steps. 
Let us put $\varphi_{0}:=\widehat{\Phi}_{0}$ and $\varphi_{k}:=\widehat{\Phi}_{k}$ for $k \in \mathbb{N}$. We can find a pair of functions $\lambda_{0}, \lambda \in \mathcal{S}\left(\mathbb{R}^{d}\right)$ with $\operatorname{supp} \lambda_{0} \subset\left\{\zeta \in \mathbb{R}^{d}:|\zeta| \leqslant 2 \varepsilon\right\}$ and $\operatorname{supp} \lambda \subset\left\{\zeta \in \mathbb{R}^{d}: \varepsilon / 2 \leqslant|\zeta| \leqslant 2 \varepsilon\right\}$ such that $\sum_{k \in \mathbb{N}_{0}} \lambda_{k} \varphi_{k} \equiv 1$, where $\lambda_{k}=\lambda\left(2^{-k}\right.$.) for $k \in \mathbb{N}$. Let us shortly demonstrate how to do that. We use the special dyadic decomposition of unity given by $\eta_{0}(t)=1$ if $|t| \leqslant 4 / 3$ and $\eta_{0}(t)=0$ if $|t|>3 / 2$. We put $\eta_{k}:=\eta_{0}\left(\cdot / 2^{k}\right)-\eta_{0}\left(\cdot / 2^{k-1}\right)$ for $k \in \mathbb{N}$. Then clearly $\eta_{0}+\sum_{k=1}^{\infty} \eta_{k} \equiv 1$ and we obtain $\sum_{k \in \mathbb{N}_{0}} \lambda_{k} \varphi_{k} \equiv 1$ by defining $\lambda_{k}:=\eta_{k}(\cdot / \varepsilon) / \varphi_{k}$ for $k \in \mathbb{N}_{0}$ and $\lambda:=\lambda_{1}(2 \cdot)$.

The support of the function $\theta:=1-\sum_{k \in \mathbb{N}} \lambda_{k} \varphi_{k} \in C_{0}^{\infty}\left(\mathbb{R}^{d}\right)$ is fully contained in $M:=\{|x| \leqslant$ $3 \varepsilon / 2\}$. Due to the Tauberian conditions, $\varphi_{0}$ is positive on $M$. Inverting $\varphi_{0}$ on $M$ and extending appropriately outside, we can construct a function $\gamma \in C_{0}^{\infty}\left(\mathbb{R}^{d}\right)$, which coincides with $1 / \varphi_{0}$ on $M$. Since $\lambda_{0} \varphi_{0}=\theta$ we thus have the factorization $\lambda_{0}=\gamma \theta$.

We now put $\lambda_{0, u}(\cdot):=\gamma(\cdot) \theta(u \cdot)$ for $u \in[1,2]$, which gives

$$
\lambda_{0, u} \varphi_{0}+\sum_{k \in \mathbb{N}} \lambda_{k}(u \cdot) \varphi_{k}(u \cdot)=1 .
$$

We then define $\Xi, \Theta, \Lambda, \Lambda_{0, u}$, and $\Lambda_{k}$ for $k \in \mathbb{N}_{0}$, all elements of $\mathcal{S}\left(\mathbb{R}^{d}\right)$, via inverse Fourier transform of the functions $\gamma, \theta, \lambda, \lambda_{0, u}$, and $\lambda_{k}$, respectively. We get $\Lambda_{0, u}=\Xi * \Theta_{u}$ and it holds $g=\Lambda_{0, u} * \Phi_{0} * g+\sum_{k \in \mathbb{N}} \Lambda_{2^{-k} u} * \Phi_{2^{-k} u} * g$ for every $g \in \mathcal{S}^{\prime}\left(\mathbb{R}^{d}\right)$.

Let $\Psi_{0}, \Psi \in \mathcal{S}\left(\mathbb{R}^{d}\right)$ be another system which satisfies the Tauberian conditions (3.5) and (3.6). Choosing $g=\Psi_{2^{-\ell} v} * f$, where $f \in \mathcal{S}^{\prime}\left(\mathbb{R}^{d}\right), \ell \in \mathbb{N}$, and $v \in[1 / 2,4]$, we get

$$
\Psi_{2^{-\ell} v} * f=\sum_{k \in \mathbb{N}} \Psi_{2^{-\ell} v} * \Lambda_{2^{-k} u} * \Phi_{2^{-k} u} * f+\Psi_{2^{-\ell} v} * \Lambda_{0, u} * \Phi_{0} * f
$$

Defining $J_{\ell, k}=\int_{\mathbb{R}^{d}}\left|\Psi_{2^{-\ell} v} * \Lambda_{2^{-k} u}(z)\right|\left(1+2^{k}|z| / u\right)^{a} d z$ for $k \in \mathbb{N}$ we have for $y \in \mathbb{R}^{d}$

$$
\begin{aligned}
\left|\left(\Psi_{2^{-\ell} v} * \Lambda_{2^{-k} u} * \Phi_{2^{-k} u} * f\right)(y)\right| & \leqslant \int_{\mathbb{R}^{d}}\left|\Psi_{2^{-\ell} v} * \Lambda_{2^{-k} u}(z)\right|\left|\Phi_{2^{-k} u} * f(y-z)\right| d z \\
& \leqslant\left(\Phi_{2^{-k} u}^{*} f\right)_{a}(y) J_{\ell, k},
\end{aligned}
$$

For $k=0$ we get with $J_{\ell, 0}=\int_{\mathbb{R}^{d}}\left|\Psi_{2^{-\ell} v} * \Lambda_{0, u}(z)\right|(1+|z|)^{a} d z$

$$
\left|\left(\Psi_{2^{-\ell} v} * \Lambda_{0, u} * \Phi_{0} * f\right)(y)\right| \leqslant \int_{\mathbb{R}^{d}}\left|\Psi_{2^{-\ell} v} * \Lambda_{0, u}(z)\right|\left|\Phi_{0} * f(y-z)\right| d z \leqslant\left(\Phi_{0}^{*} f\right)_{a}(y) J_{\ell, 0} .
$$

To estimate $J_{\ell, k}$ the following identity for functions $\mu, \nu \in \mathcal{S}\left(\mathbb{R}^{d}\right)$ is used,

$$
\left(\mu_{u} * \nu_{v}\right)(x)=\frac{1}{u^{d}}\left[\mu * \nu_{v / u}\right](x / u)=\frac{1}{v^{d}}\left[\mu_{u / v} * \nu\right](x / v),
$$

valid for $u, v>0$ and $x \in \mathbb{R}^{d}$. In case $\ell \geqslant k>0$ we obtain

$$
J_{\ell, k}=\int_{\mathbb{R}^{d}}\left|\left(\Psi_{2^{k-\ell} \frac{v}{u}} * \Lambda\right)(z)\right|(1+|z|)^{a} d z \lesssim \sup _{z \in \mathbb{R}^{d}}\left|\left(\Psi_{2^{k-\ell} \frac{v}{u}} * \Lambda\right)(z)(1+|z|)^{a+d+1}\right| \lesssim 2^{(k-\ell)(R+1)}
$$

where we used [52, Lemma 1] in the last step. In case $0<\ell<k$ we estimate similarly to obtain

$$
J_{\ell, k}=\int_{\mathbb{R}^{d}}\left|\left(\Psi * \Lambda_{2^{-(k-\ell)} u / v}(z)\right)\right|\left(1+2^{k-\ell} u|z| / v\right)^{a} d z \lesssim 2^{(\ell-k)(L+1-a)}
$$


where $L$ can be chosen arbitrarily large since $\Lambda \in \mathcal{S}_{0}\left(\mathbb{R}^{d}\right)$ fulfills moment conditions for all $L \in \mathbb{N}_{0}$.

For $\ell>k=0$ we estimate as follows, taking advantage of $\Xi \in \mathcal{S}\left(\mathbb{R}^{d}\right)$,

$$
\begin{aligned}
J_{\ell, 0} & =\int_{\mathbb{R}^{d}}\left|\left(\Psi_{2^{-\ell} v} * \Theta_{u}\right) * \Xi(z)\right|(1+|z|)^{a} d z \\
& \lesssim \sup _{y \in \mathbb{R}^{d}}\left|\left(\Psi_{2^{-\ell} v} * \Theta_{u}\right)(y)(1+|y|)^{a+d+1}\right| \int_{\mathbb{R}^{d}} \int_{\mathbb{R}^{d}}|\Xi(z-y)|(1+|z-y|)^{a}(1+|y|)^{-d-1} d z d y \\
& \lesssim \sup _{y \in \mathbb{R}^{d}}\left|\left(\Psi_{2^{-\ell} v / u} * \Theta\right)(y)(1+|y|)^{a+d+1}\right| \int_{\mathbb{R}^{d}} \int_{\mathbb{R}^{d}}(1+|z|)^{-d-1}(1+|y|)^{-d-1} d z d y \lesssim 2^{-\ell(R+1)} .
\end{aligned}
$$

Using $1+t|x| \leqslant \max \{1, t\}(1+|x|)$ and $1+|x+y| / t \leqslant(1+|y| / t)(1+|x| / t)$ for $t>0$ and $x, y \in \mathbb{R}^{d}$ we further deduce for $k \in \mathbb{N}$

$$
\begin{aligned}
\left(\Phi_{2^{-k} u}^{*} f\right)_{a}(y) & \leqslant\left(\Phi_{2^{-k} u}^{*} f\right)_{a}(x)\left(1+2^{k}|x-y| / u\right)^{a} \\
& \lesssim\left(\Phi_{2^{-k} u}^{*} f\right)_{a}(x)\left(1+2^{\ell}|x-y| / v\right)^{a} \max \left\{1,2^{(k-\ell)}\right\}^{a} .
\end{aligned}
$$

and $\left(\Phi_{0}^{*} f\right)_{a}(y) \lesssim\left(\Phi_{0}^{*} f\right)_{a}(x)\left(1+2^{\ell}|x-y| / v\right)^{a}$. Altogether, we arrive - for $k \geqslant 1$ - at

$$
\sup _{y \in \mathbb{R}^{d}} \frac{\left|\left(\Psi_{2^{-\ell} v} * \Lambda_{2^{-k} u} *\left(\Phi_{2^{-k} u} * f\right)\right)(y)\right|}{\left(1+2^{\ell}|x-y| / v\right)^{a}} \lesssim\left(\Phi_{2^{-k} u}^{*} f\right)_{a}(x) \begin{cases}2^{(k-\ell)(R+1)} & : \ell \geqslant k, \\ 2^{(\ell-k)(L+1-2 a)} & : \ell<k,\end{cases}
$$

with an implicit constant independent of $u \in[1,2]$ and $v \in[1 / 2,4]$. For $k=0$ we obtain

$$
\sup _{y \in \mathbb{R}^{d}} \frac{\left|\left(\Psi_{2^{-\ell}} * \Lambda_{0, u} * \Phi_{0} * f\right)(y)\right|}{\left(1+2^{\ell}|x-y| / v\right)^{a}} \lesssim\left(\Phi_{0}^{*} f\right)_{a}(x) 2^{-\ell(R+1)} .
$$

We thus conclude from (3.8) that uniformly in $t, u \in[1,2]$

$$
\begin{aligned}
\left\langle\Psi_{2^{-\ell} t}^{*} f\right\rangle_{a}(x) & =\sup _{t / 2 \leqslant v \leqslant 2 t, v<1}\left(\Psi_{2^{-\ell} v}^{*} f\right)_{a}(x) \\
& \lesssim\left(\Phi_{0}^{*} f\right)_{a}(x) 2^{-\ell(R+1)}+\sum_{k \in \mathbb{N}}\left(\Phi_{2^{-k} u}^{*} f\right)_{a}(x) \begin{cases}2^{(k-\ell)(R+1)} & : \ell \geqslant k, \\
2^{(\ell-k)(L+1-2 a)} & : \ell<k .\end{cases}
\end{aligned}
$$

Writing $\tilde{w}_{\ell, t}(x)=w\left(x, 2^{-\ell} t\right)$ for $\ell \in \mathbb{N}$ and $\tilde{w}_{0, t}(x)=w(x, \infty)$ we have

$$
\tilde{w}_{\ell, t}(x) \tilde{w}_{k, u}(x)^{-1} \lesssim \begin{cases}2^{(\ell-k) \alpha_{2}} & \ell \geqslant k, \\ 2^{(\ell-k) \alpha_{1}} & \ell<k,\end{cases}
$$

as a consequence of (W1), (3.2), and (3.3). Multiplying both sides with $w\left(x, 2^{-\ell} t\right)$ we finally derive with an implicit constant independent of $t, u \in[1,2]$

$$
\begin{aligned}
w\left(x, 2^{-\ell} t\right)\left\langle\Psi_{2^{-\ell} t}^{*} f\right\rangle_{a}(x) & \lesssim w(x, \infty)\left(\Phi_{0}^{*} f\right)_{a}(x) 2^{-\ell\left(R+1-\alpha_{2}\right)} \\
& +\sum_{k \in \mathbb{N}} w\left(x, 2^{-k} u\right)\left(\Phi_{2^{-k} u}^{*} f\right)_{a}(x) \begin{cases}2^{(k-\ell)\left(R+1-\alpha_{2}\right)} & : \ell \geqslant k, \\
2^{(\ell-k)\left(L+1-2 a+\alpha_{1}\right)} & : \quad \ell<k\end{cases}
\end{aligned}
$$


Choosing $L \geqslant 2 a-\alpha_{1}$ we have with $0<\delta=\min \left\{1, R+1-\alpha_{2}\right\}$ the central estimate

$$
\left\langle\Psi_{2^{-\ell} t}^{*} f\right\rangle_{a}(x) \lesssim 2^{-\ell \delta} \frac{w(x, \infty)}{w\left(x, 2^{-\ell} t\right)}\left(\Phi_{0}^{*} f\right)_{a}(x)+\sum_{k \in \mathbb{N}} 2^{-|k-\ell| \delta} \frac{w\left(x, 2^{-k} u\right)}{w\left(x, 2^{-\ell} t\right)}\left(\Phi_{2^{-k} u}^{*} f\right)_{a}(x)
$$

Step 2. We show $\left\|f\left|F_{p(\cdot), q(\cdot)}^{w}\left(\mathbb{R}^{d}\right)\left\|_{1}=\right\| f\right| F_{p(\cdot), q(\cdot)}^{w}\left(\mathbb{R}^{d}\right)\right\|_{2,3}$. The direction $\left\|f \mid F_{p(\cdot), q(\cdot)}^{w}\left(\mathbb{R}^{d}\right)\right\|_{1} \lesssim$ $\left\|f \mid F_{p(\cdot), q(\cdot)}^{w}\left(\mathbb{R}^{d}\right)\right\|_{2,3}$ is obvious and it remains to verify $\left\|f\left|F_{p(\cdot), q(\cdot)}^{w}\left(\mathbb{R}^{d}\right)\left\|_{3} \lesssim\right\| f\right| F_{p(\cdot), q(\cdot)}^{w}\left(\mathbb{R}^{d}\right)\right\|_{1}$.

We use (3.9) with $\Psi=\Phi$. Choosing $0<\tilde{\delta} \leqslant \delta$ we obtain for any $r>0$, using an embedding argument if $0<r \leqslant 1$ and Hölder's inequality otherwise,

$$
\left\langle\Phi_{2^{-\ell} t}^{*} f\right\rangle_{a}^{r}(x) w^{r}\left(x, 2^{-\ell} t\right) \lesssim 2^{-\ell \tilde{\delta} r} w^{r}(x, \infty)\left(\Phi_{0}^{*} f\right)_{a}^{r}(x)+\sum_{k \in \mathbb{N}} 2^{-|k-\ell| \tilde{\delta} r} w^{r}\left(x, 2^{-k} u\right)\left(\Phi_{2^{-k}}^{*} f\right)_{a}^{r}(x) .
$$

To estimate the sum on the right hand side we use (2.66) proved in Substep 1.3 of the proof of [59. Thm. 2.6]. It states that for $x \in \mathbb{R}^{d}, f \in \mathcal{S}^{\prime}\left(\mathbb{R}^{d}\right), k \in \mathbb{N}, u \in[1,2), r>0$, and $0<a \leqslant N$ for some arbitrary but fixed $N \in \mathbb{N}_{0}$

$$
\left(\Phi_{2^{-k} u}^{*} f\right)_{a}(x)^{r} \leqslant C_{N} \sum_{j \in \mathbb{N}_{0}} 2^{-j N r} 2^{(k+j) d} \int_{\mathbb{R}^{d}} \frac{\left|\left(\Phi_{2^{-(k+j)} u} * f\right)(y)\right|^{r}}{\left(1+2^{k}|x-y|\right)^{a r}} d y
$$

where the constant $C_{N}$ is independent of $x, f, k$, and $u \in[1,2)$, but may depend on $r, a$ and $N$. Taking into account $(W 2)$ and (3.1), which give the relation $w\left(x, 2^{-k} u\right) \lesssim 2^{-j \alpha_{1}}\left(1+2^{k} \mid x-\right.$ $y \mid)^{\alpha_{3}} w\left(y, 2^{-(j+k)} u\right)$ and $\left(1+2^{k}|z|\right)^{-M} \leqslant 2^{j M}\left(1+2^{k+j}|z|\right)^{-M}$, this leads to

$$
\begin{aligned}
&\left\langle\Phi_{2^{-\ell} t}^{*} f\right\rangle_{a}^{r}(x) w^{r}\left(x, 2^{-\ell} t\right) \lesssim 2^{-\ell \tilde{\delta} r} w^{r}(x, \infty)\left(\Phi_{0}^{*} f\right)_{a}^{r}(x) \\
&+\sum_{k \in \mathbb{N}} 2^{-|k-\ell| \tilde{\delta} r} \sum_{j \in \mathbb{N}_{0}} 2^{-j r \tilde{N}} 2^{(k+j) d} \int_{\mathbb{R}^{d}} \frac{\mid\left(\left.\Phi_{2^{-(k+j)} u^{*} f} f(y) w\left(y, 2^{-(j+k)} u\right)\right|^{r}\right.}{\left(1+2^{k+j}|x-y|\right)^{\left(a-\alpha_{3}\right) r}} d y
\end{aligned}
$$

with $\tilde{N}=N-a+\alpha_{1}+\alpha_{3}>0$. Since $x \in \mathbb{R}^{d}$ is fixed we can apply in $t$ the $L_{q(x) / r}\left([1,2) ; \frac{d t}{t}\right)$ norm with $r<\min \left\{p^{-}, q^{-}\right\}$. This changes only the constant and the left-hand side of (3.11). The $L_{q^{-} / r}\left([1,2) ; \frac{d u}{u}\right)$ (quasi-)norm in the variable $u$ only affects the right-hand side of (3.11). With Minkowski's integral inequality we obtain

$$
\begin{aligned}
& \left(\int_{1}^{2}\left|\left\langle\Phi_{2^{-\ell} t}^{*} f\right\rangle_{a}(x) w\left(x, 2^{-\ell} t\right)\right|^{q(x)} \frac{d t}{t}\right)^{r / q(x)}-2^{-\ell \tilde{\delta} r} w^{r}(x, \infty)\left(\Phi_{0}^{*} f\right)_{a}^{r}(x) \\
& \quad \lesssim \sum_{k \in \mathbb{N}} 2^{-|k-\ell| \tilde{\delta} r} \sum_{j \in \mathbb{N}_{0}} 2^{-|j-k| \tilde{N} r} 2^{j d} \int_{\mathbb{R}^{d}} \frac{\left(\int_{1}^{2}\left|\left(\Phi_{2^{-j} u} * f\right)(y) w\left(y, 2^{-j} u\right)\right|^{q^{-}} \frac{d u}{u}\right)^{r / q^{-}}}{\left(1+2^{j}|x-y|\right)^{\left(a-\alpha_{3}\right) r}} d y \\
& \quad \lesssim \sum_{k \in \mathbb{N}} 2^{-|k-\ell| \tilde{\delta} r} \sum_{j \in \mathbb{N}_{0}} 2^{-|j-k| \tilde{N} r}\left[\eta_{j,\left(a-\alpha_{3}\right) r} *\left(\int_{1}^{2}\left|\left(\Phi_{2^{-j} u} * f\right)(\cdot) w\left(\cdot, 2^{-j} u\right)\right|^{q^{-}} \frac{d u}{u}\right)^{r / q^{-}}\right](x)
\end{aligned}
$$

with functions $\eta_{\nu, m}(x)=2^{\nu d}\left(1+2^{\nu}|x|\right)^{-m}$.

Now we choose $r>0$ such that $\frac{d}{a-\alpha_{3}}<r<\min \left\{p^{-}, q^{-}\right\}$, which is possible since $a>\alpha_{3}+$ $\frac{d}{\min \left\{p^{-}, q^{-}\right\}}$, and $N$ such that $\tilde{N}>0$. Applying the $L_{p(\cdot) / r}\left(\ell_{q(\cdot) / r}\right)$ norm with respect to $x \in \mathbb{R}^{d}$ 
and $\ell \in \mathbb{N}$ and using Lemma 3.12 twice together with Lemma 3.10 (note $\left(a-\alpha_{3}\right) r>d$ ) then yields

$$
\begin{aligned}
& \left\|\left(\int_{1}^{2}\left|\left\langle\Phi_{2^{-\ell} t}^{*} f\right\rangle_{a}(\cdot) w\left(\cdot, 2^{-\ell} t\right)\right|^{q(\cdot)} \frac{d t}{t}\right)^{r / q(\cdot)}\left|L_{p(\cdot) / r}\left(\ell_{q(\cdot) / r}\right)\|-c\| w(\cdot, \infty)\left(\Phi_{0}^{*} f\right)_{a}(\cdot)\right| L_{p(\cdot)}\left(\mathbb{R}^{d}\right)\right\|^{r} \\
& \quad \lesssim\left\|\left[\eta_{\ell,\left(a-\alpha_{3}\right) r} *\left(\int_{1}^{2}\left|\left(\Phi_{2^{-\ell} u} * f\right)(\cdot) w\left(\cdot, 2^{-\ell} u\right)\right|^{q^{-}} \frac{d u}{u}\right)^{r / q^{-}}\right](x) \mid L_{p(\cdot) / r}\left(\ell_{q(\cdot) / r}\right)\right\| \\
& \quad \lesssim\left\|\left(\int_{1}^{2}\left|\left(\Phi_{2^{-\ell} u} * f\right)(\cdot) w\left(\cdot, 2^{-\ell} u\right)\right|^{q^{-}} \frac{d u}{u}\right)^{r / q^{-}} \mid L_{p(\cdot) / r}\left(\ell_{q(\cdot) / r}\right)\right\|
\end{aligned}
$$

Finally, we use Hölder's inequality to estimate the integral in the last norm. We use $0<q^{-} \leqslant$ $q(x)$ and get

$$
\begin{aligned}
\left(\int_{1}^{2} \mid\left(\Phi_{2^{-\ell} u}\right.\right. & \left.* f)\left.(x) w\left(x, 2^{-\ell} u\right)\right|^{q^{-}} \frac{d u}{u}\right)^{r / q^{-}} \\
& \leqslant\left(\int_{1}^{2}\left|\left(\Phi_{2^{-\ell} u} * f\right)(x) w\left(x, 2^{-\ell} u\right)\right|^{q(x)} \frac{d u}{u}\right)^{r / q(x)}\left(\int_{1}^{2} \frac{d u}{u}\right)^{r / q^{-} \cdot \frac{1}{\left(\frac{q(x)}{q^{-}}\right)^{\prime}}} \\
& \leqslant\left(\int_{1}^{2}\left|\left(\Phi_{2^{-\ell} u} * f\right)(x) w\left(x, 2^{-\ell} u\right)\right|^{q(x)} \frac{d u}{u}\right)^{r / q(x)} .
\end{aligned}
$$

Using this estimate we can reformulate (3.12) into

$$
\begin{aligned}
& \left\|\left(\int_{0}^{1}\left|\left\langle\Phi_{\lambda}^{*} f\right\rangle_{a}(\cdot) w(\cdot, \lambda)\right|^{q(\cdot)} \frac{d \lambda}{\lambda}\right)^{1 / q(\cdot)} \mid L_{p(\cdot)}\right\| \\
& \quad \lesssim\left\|w(\cdot, \infty)\left(\Phi_{0}^{*} f\right)_{a}(\cdot)\left|L_{p(\cdot)}\left(\mathbb{R}^{d}\right)\|+\|\left(\int_{0}^{1}\left|\left(\Phi_{\lambda} * f\right)(\cdot) w(\cdot, \lambda)\right|^{q(\cdot)} \frac{d \lambda}{\lambda}\right)^{1 / q(\cdot)}\right| L_{p(\cdot)}\right\| .
\end{aligned}
$$

The inhomogeneous term $\left(\Phi_{0}^{*} f\right)_{a}(x)$ needs to be treated separately. The argumentation, however, is analogous to the exposition before with (3.10) replaced by the inequality

$$
\left(\Phi_{0}^{*} f\right)_{a}(x)^{r} \lesssim \sum_{k \in \mathbb{N}} 2^{-k N r} 2^{k d} \int_{\mathbb{R}^{d}} \frac{\left|\left(\Phi_{2^{-k} u} * f\right)(y)\right|^{r}}{(1+|x-y|)^{a r}} d y+\int_{\mathbb{R}^{d}} \frac{\left|\left(\Phi_{0} * f\right)(y)\right|^{r}}{(1+|x-y|)^{a r}} d y .
$$

In the Besov space case we do not need the functions $\eta_{\nu, m}$ and one can work with the usual maximal operator $\mathcal{M}$ together with Lemma 3.3, see [36] for details.

Step 3. In the third step we show $\left\|f\left|F_{p(\cdot), q(\cdot)}^{w}\left(\mathbb{R}^{d}\right)\left\|_{2}=\right\| f\right| F_{p(\cdot), q(\cdot)}^{w}\left(\mathbb{R}^{d}\right)\right\|_{3}=\left\|f \mid F_{p(\cdot), q(\cdot)}^{w}\left(\mathbb{R}^{d}\right)\right\|_{4}$. We immediately observe $\left\|f\left|F_{p(\cdot), q(\cdot)}^{w}\left(\mathbb{R}^{d}\right)\left\|_{2} \lesssim\right\| f\right| F_{p(\cdot), q(\cdot)}^{w}\left(\mathbb{R}^{d}\right)\right\|_{3}$.

Substep 3.1. To prove $\left\|f\left|F_{p(\cdot), q(\cdot)}^{w}\left(\mathbb{R}^{d}\right)\left\|_{3} \lesssim\right\| f\right| F_{p(\cdot), q(\cdot)}^{w}\left(\mathbb{R}^{d}\right)\right\|_{4}$ we apply (3.9) with $u=1$ and $\Psi=\Phi$. Since the inhomogeneous terms are identical, it suffices to estimate the homogeneous part. Integration with respect to $d t / t$ yields for $\ell \in \mathbb{N}$

$$
\left(\int_{1}^{2}\left|w\left(x, 2^{-\ell} t\right)\left\langle\Phi_{2^{-\ell} t}^{*} f\right\rangle_{a}(x)\right|^{q(x)} \frac{d t}{t}\right)^{\frac{1}{q(x)}} \lesssim 2^{-\ell \delta} w_{0}(x)\left(\Phi_{0}^{*} f\right)_{a}(x)+\sum_{k \in \mathbb{N}} 2^{-|k-\ell| \delta} w_{k}(x)\left(\Phi_{2^{-k}}^{*} f\right)_{a}(x) .
$$


Let us denote the function on the right-hand side of the previous estimate by $G_{\ell}$. Applying the vector-valued convolution inequality of Lemma 3.12 then proves

$$
\begin{aligned}
& \left\|\left(\sum_{\ell=1}^{\infty} \int_{1}^{2}\left|w\left(\cdot, 2^{-\ell} t\right)\left\langle\Phi_{2^{-\ell} t}^{*} f\right\rangle_{a}(\cdot)\right|^{q(\cdot)} \frac{d t}{t}\right)^{1 / q(\cdot)}\left|L_{p(\cdot)}\|\lesssim\|\left\{G_{\ell}\right\}_{\ell \in \mathbb{N}}\right| L_{p(\cdot)}\left(\ell_{q(\cdot)}\right)\right\| \\
& \quad \lesssim\left\|w_{0}\left(\Phi_{0}^{*} f\right)_{a}\left|L_{p(\cdot)}\|+\|\left\{w_{k}\left(\Phi_{2^{-k}}^{*} f\right)_{a}\right\}_{k \in \mathbb{N}}\right| L_{p(\cdot)}\left(\ell_{q(\cdot)}\right)\right\|=\left\|f \mid F_{p(\cdot), q(\cdot)}^{w}\right\|_{4} .
\end{aligned}
$$

Substep 3.2: Let us finish by proving $\left\|f\left|F_{p(\cdot), q(\cdot)}^{w}\left(\mathbb{R}^{d}\right)\left\|_{4} \lesssim\right\| f\right| F_{p(\cdot), q(\cdot)}^{w}\left(\mathbb{R}^{d}\right)\right\|_{2}$. Again it suffices to estimate the homogeneous part. For this we let $t=1$ and $\Psi=\Phi$ in (3.9). If $q(x) \geqslant 1$ we can use Minkowski's inequality to deduce

$$
\begin{aligned}
w_{\ell}(x)\left(\Phi_{2^{-\ell}}^{*} f\right)_{a}(x) & \lesssim 2^{-\ell \delta} w(x, \infty)\left(\Phi_{0}^{*} f\right)_{a}(x) \\
& +\sum_{k \in \mathbb{N}} 2^{-|k-\ell| \delta}\left(\int_{1}^{2}\left|\tilde{w}_{k, u}(x)\left(\Phi_{2^{-k} u}^{*} f\right)_{a}(x)\right|^{q(x)} \frac{d u}{u}\right)^{1 / q(x)} .
\end{aligned}
$$

Applying the $\ell_{q(x)}$-norm on both sides, Young's convolution inequality then yields

$$
\begin{aligned}
\sum_{\ell \in \mathbb{N}} w_{\ell}(x)^{q(x)}\left(\Phi_{2^{-\ell}}^{*} f\right)_{a}(x)^{q(x)} & \lesssim\left(w(x, \infty)\left(\Phi_{0}^{*} f\right)_{a}(x)\right)^{q(x)} \\
& +\sum_{k \in \mathbb{N}} \int_{1}^{2}\left|\tilde{w}_{k, u}(x)\left(\Phi_{2^{-k} u}^{*} f\right)_{a}(x)\right|^{q(x)} \frac{d u}{u} .
\end{aligned}
$$

If $q(x)<1$ we use the $q(x)$-triangle inequality

$$
\begin{aligned}
\left(w_{\ell}(x)\left(\Phi_{2^{-\ell}}^{*} f\right)_{a}(x)\right)^{q(x)} & \lesssim 2^{-\ell \delta q(x)}\left(w(x, \infty)\left(\Phi_{0}^{*} f\right)_{a}(x)\right)^{q(x)} \\
& +\sum_{k \in \mathbb{N}} 2^{-|k-\ell| q(x) \delta} \int_{1}^{2}\left|\tilde{w}_{k, u}(x)\left(\Phi_{2^{-k} u}^{*} f\right)_{a}(x)\right|^{q(x)} \frac{d u}{u} .
\end{aligned}
$$

Now we take on both sides the $\ell_{1}$-norm with respect to the index $\ell \in \mathbb{N}$ and take into account $\sum_{k \in \mathbb{N}_{0}} 2^{-|k| q(x) \delta} \leqslant C$. We thus arrive at the same estimate (3.13). Taking the $L_{p(\cdot) \text {-quasi-norm }}$ of (3.13) finishes the proof of Substep 3.2 and hence Step 3.

Step 4: Relation (3.9) also immediately allows to change to a different system $\Psi_{0}, \Psi$, however in the discrete setting the change of systems has already been shown in [36].

Remark 3.13. The previous theorem ensures in particular the independence of Besov-LizorkinTriebel type spaces with variable exponents from the chosen resolution of unity if $p, q \in \mathcal{P}^{\log }\left(\mathbb{R}^{d}\right)$ with $p^{+}<\infty, q^{+}<\infty$ in the $F$-case and $p \in \mathcal{P}^{\log }\left(\mathbb{R}^{d}\right), \tilde{q} \in(0, \infty]$ in the $B$-case.

\section{Variable exponent spaces as coorbits}

In order to treat the spaces $B_{p(\cdot), \tilde{q}^{w}}^{w}\left(\mathbb{R}^{d}\right)$ and $F_{p(\cdot), q(\cdot)}^{w}\left(\mathbb{R}^{d}\right)$ as coorbits we utilize an inhomogeneous version of the continuous wavelet transform, which uses high scale wavelets together with a base scale for the analysis. The corresponding index set is $X=\mathbb{R}^{d} \times[(0,1) \cup\{\infty\}]$, where $\infty$ denotes an isolated point, equipped with the Radon measure $\mu$ defined by

$$
\int_{X} F(\mathbf{x}) d \mu(\mathbf{x})=\int_{\mathbb{R}^{d}} \int_{0}^{1} F(x, s) \frac{d s}{s^{d+1}} d x+\int_{\mathbb{R}^{d}} F(x, \infty) d x .
$$


The wavelet transform is then given by $V_{\mathcal{F}} f(\mathbf{x})=\left\langle f, \varphi_{\mathbf{x}}\right\rangle, \mathbf{x} \in X$, for a continuous frame $\mathcal{F}=\left\{\varphi_{\mathbf{x}}\right\}_{\mathbf{x} \in X}$ on $\mathcal{H}=L_{2}\left(\mathbb{R}^{d}\right)$ of the form

$$
\varphi_{(x, \infty)}=T_{x} \Phi_{0}=\Phi_{0}(\cdot-x) \quad \text { and } \quad \varphi_{(x, t)}=T_{x} \mathcal{D}_{t}^{L_{2}} \Phi=t^{-d / 2} \Phi((\cdot-x) / t),
$$

with suitable functions $\Phi_{0}, \Phi \in L_{2}\left(\mathbb{R}^{d}\right)$. Such a frame $\mathcal{F}=\mathcal{F}\left(\Phi_{0}, \Phi\right)$ will in our context be referred to as a continuous wavelet frame in $L_{2}\left(\mathbb{R}^{d}\right)$.

Definition 4.1. A continuous wavelet frame $\mathcal{F}=\mathcal{F}\left(\Phi_{0}, \Phi\right)$ is admissible if $\Phi_{0} \in \mathcal{S}\left(\mathbb{R}^{d}\right)$ and $\Phi \in \mathcal{S}_{0}\left(\mathbb{R}^{d}\right)$ are chosen such that they satisfy the Tauberian conditions (3.5), (3.6) and the condition

$$
\left|\widehat{\Phi}_{0}(\xi)\right|^{2}+\int_{0}^{1}|\widehat{\Phi}(t \xi)|^{2} \frac{d t}{t}=C \quad \text { for a.e. } \xi \in \mathbb{R}^{d} .
$$

An admissible wavelet frame $\mathcal{F}\left(\Phi_{0}, \Phi\right)$ represents a tight continuous frame in the sense of (1.1). To see this, apply Fubini's and Plancherel's theorem to get

$$
C\left\|\left.f\left|L_{2}\left(\mathbb{R}^{d}\right) \|^{2}=\int_{\mathbb{R}^{d}}\right| \widehat{f}(\xi)\right|^{2}\left(\left|\widehat{\Phi}_{0}(\xi)\right|^{2}+\int_{0}^{1}|\widehat{\Phi}(t \xi)|^{2} \frac{d t}{t}\right) d \xi=(2 \pi)^{-d} \int_{X}\left|\left\langle f, \varphi_{\mathbf{x}}\right\rangle\right|^{2} d \mu(\mathbf{x}) .\right.
$$

\subsection{Peetre-Wiener type spaces on $X$}

We intend to define two general scales of spaces on $X$, for which we need a Peetre type maximal function, given for a measurable function $F: X \rightarrow \mathbb{C}$ by

$$
\begin{gathered}
\mathcal{P}_{a}^{*} F(x, t):=\operatorname{ess}_{\substack{z \in \mathbb{R}^{d}, \tau<1 \\
\frac{t}{2} \leqslant \tau \leqslant 2 t}} \frac{|F(x+z, \tau)|}{(1+|z| / \tau)^{a}} \quad, \quad x \in \mathbb{R}^{d}, 0<t<1, \\
\mathcal{P}_{a}^{*} F(x, \infty):=\underset{z \in \mathbb{R}^{d}}{\operatorname{ess} \sup } \frac{|F(x+z, \infty)|}{(1+|z|)^{a}} \quad, \quad x \in \mathbb{R}^{d} .
\end{gathered}
$$

The operator $\mathcal{P}_{a}^{*}$ is a stronger version of the usual Peetre maximal operator $\mathcal{P}_{a}$, which does not take the supremum over $t$ and was used e.g. in [50].

Definition 4.2. Let $p, q \in \mathcal{P}^{\log }\left(\mathbb{R}^{d}\right)$ with $0<p^{-} \leqslant p^{+}<\infty$ and $0<q^{-} \leqslant q^{+}<\infty$ and let $0<\tilde{q} \leqslant \infty$. Further, let $a>0$ and $w \in \mathcal{W}_{\alpha_{1}, \alpha_{2}}^{\alpha_{3}}$. Then we define by

$$
\begin{aligned}
P_{p(\cdot), q(\cdot), a}^{w}(X) & =\left\{F: X \rightarrow \mathbb{C}:\left\|F \mid P_{p(\cdot), q(\cdot), a}^{w}\right\|<\infty\right\} \\
L_{p(\cdot), \tilde{q}, a}^{w}(X) & =\left\{F: X \rightarrow \mathbb{C}:\left\|F \mid L_{p(\cdot), \tilde{q}, a}^{w}\right\|<\infty\right\}
\end{aligned}
$$

two scales of function spaces on $X$ with respective quasi-norms

$$
\begin{aligned}
\left\|F \mid P_{p(\cdot), q(\cdot), a}^{w}\right\|:= & \left\|w(\cdot, \infty) \mathcal{P}_{a}^{*} F(\cdot, \infty) \mid L_{p(\cdot)}\left(\mathbb{R}^{d}\right)\right\| \\
& +\left\|\left(\int_{0}^{1}\left[w(\cdot, t) \mathcal{P}_{a}^{*} F(\cdot, t)\right]^{q(\cdot)} \frac{d t}{t}\right)^{1 / q(\cdot)} \mid L_{p(\cdot)}\left(\mathbb{R}^{d}\right)\right\|, \\
\left\|F \mid L_{p(\cdot), \tilde{q}, a}^{w}\right\|:= & \left.\| w(\cdot, \infty) \mathcal{P}_{a}^{*} F(\cdot, \infty) \mid L_{p(\cdot)} \mathbb{R}^{d}\right) \| \\
& +\left(\int_{0}^{1}\left\|w(\cdot, t) \mathcal{P}_{a}^{*} F(\cdot, t) \mid L_{p(\cdot)}\left(\mathbb{R}^{d}\right)\right\| \frac{\tilde{q}}{d t} \frac{1}{t}\right)^{1 / \tilde{q}} .
\end{aligned}
$$


It is not hard to verify that in case $a>d / p^{-}+\alpha_{3}$ these spaces are rich solid QBF-spaces as defined and studied in Subsection 2.1. Moreover, the utilization of the Peetre-Wiener operator $\mathcal{P}_{a}^{*}$ ensures that they are locally integrable, even in the quasi-Banach case in contrast to the ordinary Peetre spaces where $\mathcal{P}_{a}$ is used instead of $\mathcal{P}_{a}^{*}$. In fact, there is an associated locally bounded weight function given by

$$
\nu_{w, p(\cdot), q(\cdot)}(x, t)=\left\{\begin{aligned}
t^{\alpha_{1}-d / p^{-}}(1+|x|)^{\alpha_{3}} & , \quad x \in \mathbb{R}^{d}, 0<t<1, \\
(1+|x|)^{\alpha_{3}} & , \quad x \in \mathbb{R}^{d}, t=\infty
\end{aligned}\right.
$$

such that the following lemma holds true.

Lemma 4.3. We have the continuous embeddings

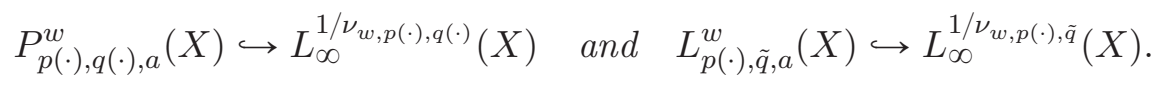

Proof. It is useful to interpret the component $\mathbb{R}^{d} \times(0,1)$ of the index $X$ as a subset of the $a x+b$ group $\mathcal{G}=\mathbb{R}^{d} \times(0, \infty)$ with multiplication $(x, t)(y, s)=(x+t y, t s)$ and $(x, t)^{-1}=$ $(-x / t, 1 / t)$. Let $U^{-1}$ be the inversion of $U:=[-2,2]^{d} \times\left[\frac{1}{2}, 2\right]$ and define $U_{(x, t)}:=(x, t) U^{-1}$ and $\widetilde{U}_{(x, t)}:=(x, t) U$. Further put $Q_{(x, t)}:=x+t[-1,1]^{d}$ and $U_{(x, \infty)}:=\widetilde{U}_{(x, \infty)}:=Q_{(x, 1)} \times\{\infty\}$. Then we can estimate for $F: X \rightarrow \mathbb{C}$ and almost all $(x, t) \in X$ at every fixed $(y, s) \in X$

$$
|F(x, t)| \chi_{U_{(x, t)}}(y, s) \leqslant \operatorname{essiup}_{(x, t) \in X \cap \widetilde{U}_{(y, s)}}|F(x, t)| \lesssim \mathcal{P}_{a}^{*} F(y, s) .
$$

For convenience, let us introduce

$$
\left\|F\left|M_{p(\cdot), q(\cdot)}^{w}\|:=\| w(\cdot, \infty) F(\cdot, \infty)\right| L_{p(\cdot)}\right\|+\left\|\left(\int_{0}^{1}|w(\cdot, t) F(\cdot, t)|^{q(\cdot)} \frac{d t}{t}\right)^{1 / q(\cdot)} \mid L_{p(\cdot)}\right\| .
$$

We obtain for almost all $(x, t) \in X$

$$
|F(x, t)| \cdot\left\|\chi_{U_{(x, t)}}\left|M_{p(\cdot), q(\cdot)}^{w}\|\lesssim\| \mathcal{P}_{a}^{*} F\right| M_{p(\cdot), q(\cdot)}^{w}\right\|=\left\|F \mid P_{p(\cdot), q(\cdot), a}^{w}\right\| \cdot
$$

It remains to prove $\nu_{w, p(\cdot), q(\cdot)}(x, t) \gtrsim\left\|\chi_{U_{(x, t)}} \mid M_{p(\cdot), q(\cdot)}^{w}\right\|^{-1}$. Since $U^{-1} \supset[-1,1]^{d} \times\left[\frac{1}{2}, 2\right]$ we have $U_{(x, t)} \supset Q_{(x, t)} \times\left[\frac{t}{2}, 2 t\right]$. If $0<t<1$ it follows for $x, y \in \mathbb{R}^{d}$

$$
\left(\int_{0}^{1}\left[w(y, s) \chi_{U_{(x, t)}}(y, s)\right]^{q(y)} \frac{d s}{s}\right)^{1 / q(y)} \gtrsim \ln (4)^{1 / q(y)} w(y, t) \chi_{Q_{(x, t)}}(y) \gtrsim w(y, t) \chi_{Q_{(x, t)}}(y)
$$

and $\chi_{U_{(x, t)}}(y, \infty)=0$. The properties (W1) and (W2) of $w \in \mathcal{W}_{\alpha_{1}, \alpha_{2}}^{\alpha_{3}}$ further imply

$$
w(y, t) \gtrsim w(x, t)(1+|x-y| / t)^{-\alpha_{3}} \gtrsim t^{-\alpha_{1}}(1+|x|)^{-\alpha_{3}}(1+|x-y| / t)^{-\alpha_{3}} .
$$

This leads to $\quad\left\|\chi_{U_{(x, t)}}\left|M_{p(\cdot), q(\cdot)}^{w}\left\|\gtrsim t^{-\alpha_{1}}(1+|x|)^{-\alpha_{3}}\right\| \chi_{Q_{(x, t)}}(\cdot)(1+|x-\cdot| / t)^{-\alpha_{3}}\right| L_{p(\cdot)}\right\|$. Since $\left\|\chi_{Q_{(x, t)}} \mid L_{p(\cdot)}\right\| \geqslant \frac{1}{2} \min \left\{\left|Q_{(x, t)}\right|^{1 / p^{+}},\left|Q_{(x, t)}\right|^{1 / p^{-}}\right\}$by [16, Lemma 3.2.12] and $\left|Q_{(x, t)}\right|=(2 t)^{d}$ we obtain $\left\|\chi_{Q_{(x, t)}} \mid L_{p(\cdot)}\right\| \gtrsim t^{d / p^{-}}$and finally arrive at

$$
\left\|\chi_{U_{(x, t)}}\left|M_{p(\cdot), q(\cdot)}^{w}\left\|\gtrsim t^{-\alpha_{1}}(1+|x|)^{-\alpha_{3}}\right\| \chi_{Q_{(x, t)}}\right| L_{p(\cdot)}\left(\mathbb{R}^{d}\right)\right\| \gtrsim\left(\nu_{w, p(\cdot), q(\cdot)}(x, t)\right)^{-1},
$$

where $\chi_{Q_{(x, t)}}(y)(1+|x-y| / t)^{-\alpha_{3}}=\chi_{Q_{(x, t)}}(y)$ was used. If $t=\infty$ we can argue analogously. 


\subsection{Coorbit identification}

As the following lemma shows, every admissible wavelet frame $\mathcal{F}=\mathcal{F}\left(\Phi_{0}, \Phi\right)$ in the sense of Definition 4.1 is suitable for the definition of coorbits of Peetre-Wiener spaces.

Standing assumptions: For the rest of the paper the indices fulfill $p, q \in \mathcal{P}^{\log }\left(\mathbb{R}^{d}\right)$ with $0<p^{-} \leqslant p^{+}<\infty, 0<q^{-} \leqslant q^{+}<\infty$. Further $\tilde{q} \in(0, \infty]$ and $w \in \mathcal{W}_{\alpha_{1}, \alpha_{2}}^{\alpha_{3}}$ for arbitrary but fixed $\alpha_{2} \geqslant \alpha_{1}$ and $\alpha_{3} \geqslant 0$.

Lemma 4.4. An admissible continuous wavelet frame $\mathcal{F}$ in the sense of (4.1) with generators $\Phi_{0} \in \mathcal{S}\left(\mathbb{R}^{d}\right)$ and $\Phi \in \mathcal{S}_{0}\left(\mathbb{R}^{d}\right)$ has property $F(\nu, Y)$ for $Y=P_{p(\cdot), q(\cdot), a}^{w}(X)$ and $Y=L_{p(\cdot), \tilde{q}, a}^{w}(X)$, and where $\nu=\nu_{w, p(\cdot), q(\cdot)}$ is the corresponding weight from (4.2).

Proof. The proof goes along the lines of [50, Lem. 4.18]. The kernel estimates in [50, Lem. $4.8,4.24]$ have to be adapted to the Peetre-Wiener space. This is a straight-forward procedure and allows for treating as well the quasi-Banach situation .

Now we are ready for the coorbit characterization of $B_{p(\cdot), \tilde{q}^{w}}^{w}\left(\mathbb{R}^{d}\right)$ and $F_{p(\cdot), q(\cdot)}^{w}\left(\mathbb{R}^{d}\right)$. Note that the weight $\tilde{w}$ defined in (4.4) is an element of the class $\mathcal{W}_{\alpha_{1}+d / 2, \alpha_{2}+d / 2}^{\alpha_{3}}$.

Theorem 4.5. Let $p(\cdot), q(\cdot), \tilde{q}, w$ fulfill the standing assumptions (4.3). We choose an admissible continuous wavelet frame $\mathcal{F}=\mathcal{F}\left(\Phi_{0}, \Phi\right)$ according to Definition 4.1. Putting

$$
\tilde{w}(x, t):=\left\{\begin{aligned}
t^{-d / 2} w(x, t) & , \quad 0<t<1, \\
w(x, \infty) & , \quad t=\infty
\end{aligned}\right.
$$

we have $B_{p(\cdot), \tilde{q}}^{w}\left(\mathbb{R}^{d}\right)=\operatorname{Co}\left(\mathcal{F}, L_{p(\cdot), \tilde{q}, a}^{\tilde{w}}\right)$ if $a>\frac{d}{p^{-}}+\alpha_{3}$ and $F_{p(\cdot), q(\cdot)}^{w}\left(\mathbb{R}^{d}\right)=\operatorname{Co}\left(\mathcal{F}, P_{p(\cdot), q(\cdot), a}^{\tilde{w}}\right)$ if $a>\max \left\{\frac{d}{p^{-}}, \frac{d}{q^{-}}\right\}+\alpha_{3}$ in the sense of equivalent quasi-norms.

Proof. By Lemma 4.4 the coorbits exist in accordance with the theory. Now, let $f \in \mathcal{S}\left(\mathbb{R}^{d}\right)$ and $F(x, t):=V_{\mathcal{F}} f(x, t)=\left\langle f, \varphi_{(x, t)}\right\rangle$ with $\varphi_{(x, t)}$ as in 44.1). According to [59, Lem. A.3]

$$
\left|V_{\mathcal{F}} f(x, t)\right| \leqslant C_{N}(f) G_{N}(x, t) \quad \text { with } \quad G_{N}(x, t)= \begin{cases}t^{N}(1+|x|)^{-N} & , 0<t<1 \\ (1+|x|)^{-N} & , t=\infty\end{cases}
$$

where $N \in \mathbb{N}$ is arbitrary but fixed and $C_{N}(f)>0$ is a constant depending on $N$ and $f$. Choosing $N$ large, we have $G_{N} \in L_{1}^{\nu}(X)$ and thus $F \in L_{1}^{\nu}(X)$ with $\left\|F\left|L_{1}^{\nu}\left\|\leqslant C_{N}(f)\right\| G_{N}\right| L_{1}^{\nu}\right\|$. This proves $f \in \mathcal{H}_{1}^{\nu}$. Even more, given a sequence $\left(f_{n}\right)_{n \in \mathbb{N}} \subset \mathcal{S}\left(\mathbb{R}^{d}\right)$ we have $C_{N}\left(f_{n}\right) \rightarrow 0$ if $f_{n} \rightarrow 0$ in $\mathcal{S}\left(\mathbb{R}^{d}\right)$. This is due to the fact that the constants $C_{N}\left(f_{n}\right)$ can be estimated by the Schwartz semi-norms of $f_{n}$ up to order $N$ (see proof of [59, Lem. A.3]). Hence, $\mathcal{F} \subset \mathcal{S}\left(\mathbb{R}^{d}\right) \hookrightarrow \mathcal{H}_{1}^{\nu}$ and the voice transform $V_{\mathcal{F}}$ extends to $\mathcal{S}^{\prime}\left(\mathbb{R}^{d}\right)$. Moreover, by a straight-forward modification of the argument in [34, Cor. 20.0.2], the reproducing formula is still valid on $\mathcal{S}^{\prime}\left(\mathbb{R}^{d}\right)$. Therefore we may apply Lemma 2.28 and use the larger reservoir $\mathcal{S}^{\prime}\left(\mathbb{R}^{d}\right)$.

To see that the coorbits coincide with $B_{p(\cdot), \tilde{q}}^{w}\left(\mathbb{R}^{d}\right)$ and $F_{p(\cdot), q(\cdot)}^{w}\left(\mathbb{R}^{d}\right)$, note that the functions $\tilde{\Phi}=\overline{\Phi(-\cdot)}$ and $\tilde{\Phi}_{0}=\overline{\Phi_{0}(-\cdot)}$ satisfy the Tauberian conditions (3.5), (3.6) and can thus be used in the continuous characterization of Theorem 3.11. Recall the notation $\tilde{\Phi}_{t}=t^{-d} \tilde{\Phi}(\cdot / t)$. The assertion is now a direct consequence of the possible reformulation $\left(V_{\mathcal{F}} f\right)(\cdot, \infty)=\tilde{\Phi}_{0} * f$ and

$$
\left(V_{\mathcal{F}} f\right)(x, t)=\left(\mathcal{D}_{t}^{L_{2}} \overline{\Phi(-\cdot)} * f\right)(x)=t^{d / 2}\left(\tilde{\Phi}_{t} * f\right)(x) \quad, 0<t<1, x \in \mathbb{R}^{d} .
$$




\subsection{Atomic decompositions and quasi-Banach frames}

Based on the coorbit characterizations of Theorem 4.5 we can now apply the abstract theory from Section 2 in our concrete setup, in particular the discretization machinery. We will subsequently use the following covering of the space $X$. For $\alpha>0$ and $\beta>1$ we consider the family $\mathcal{U}^{\alpha, \beta}=\left\{U_{j, k}\right\}_{j \in \mathbb{N}_{0}, k \in \mathbb{Z}^{d}}$ of subsets

$$
\begin{aligned}
U_{0, k} & =Q_{0, k} \times\{\infty\} \quad, \quad k \in \mathbb{Z}^{d}, \\
U_{j, k} & =Q_{j, k} \times\left[\beta^{-j}, \beta^{-j+1}\right) \quad, \quad j \in \mathbb{N}, k \in \mathbb{Z}^{d},
\end{aligned}
$$

where $Q_{j, k}=\alpha \beta^{-j} k+\alpha \beta^{-j}[0,1]^{d}$. Clearly, we have $X \subset \bigcup_{j \in \mathbb{N}_{0}, k \in \mathbb{Z}^{d}} U_{j, k}$ and $\mathcal{U}=\mathcal{U}^{\alpha, \beta}$ is an admissible covering of $X$.

The abstract Theorem 2.48 provides atomic decompositions for $B_{p(\cdot), \tilde{q}^{w}}^{w}\left(\mathbb{R}^{d}\right)$ and $F_{p(\cdot), q(\cdot)}^{w}\left(\mathbb{R}^{d}\right)$. To apply it we need to analyze the oscillation kernels $\operatorname{osc}_{\alpha, \beta}:=\operatorname{osc}_{\mathcal{U}, \Gamma}$ and $\operatorname{osc}_{\alpha, \beta}^{*}:=\operatorname{osc}_{\mathcal{U}, \Gamma}^{*}$, where we choose the trivial phase function $\Gamma \equiv 1$. This goes along the lines of [50, Sect. 4.4]

Proposition 4.6. Let $\mathcal{F}=\mathcal{F}\left(\Phi_{0}, \Phi\right)$ be an admissible wavelet frame, $Y=L_{p(\cdot), \tilde{q}, a}^{w}(X)$ or $Y=P_{p(\cdot), q(\cdot), a}^{w}(X)$, and $\nu=\nu_{w, p(\cdot), q(\cdot)}$ the associated weight (4.2).

(i) The kernels $\operatorname{osc}_{\alpha, \beta}$ and $\mathrm{osc}_{\alpha, \beta}^{*}$ are bounded operators on $Y$ and belong to $\mathcal{A}_{m_{\nu}}$.

(ii) If $\alpha \downarrow 0$ and $\beta \downarrow 1$ then $\left\|\operatorname{osc}_{\alpha, \beta} \mid \mathcal{B}_{Y, m_{\nu}}\right\| \rightarrow 0$ and $\left\|\operatorname{osc}_{\alpha, \beta}^{*} \mid \mathcal{B}_{Y, m_{\nu}}\right\| \rightarrow 0$.

Proof. The proof is a straight-forward modification of [50, Lem. 4.22]. Similar as in Lemma 4.4 above we have to adapt the kernel estimates to the Peetre-Wiener spaces.

Finally, Theorem 2.48 yields the following discretization result in our concrete setting, which we only state for $F_{p(\cdot), q(\cdot)}^{w}\left(\mathbb{R}^{d}\right)$ since for $B_{p(\cdot), \tilde{q}^{w}}^{w}\left(\mathbb{R}^{d}\right)$ it is essentially the same.

Theorem 4.7. Let $p(\cdot), q(\cdot), w$ fulfill the standing assumptions (4.3), assume further $a>$ $\max \left\{d / p^{-}, d / q^{-}\right\}+\alpha_{3}$ and let $\tilde{w}$ be given as in (4.4). For an admissible continuous wavelet frame $\mathcal{F}=\left\{\varphi_{\mathbf{x}}\right\}_{\mathbf{x} \in X}$ there exist $\alpha_{0}>0$ and $\beta_{0}>1$, such that for all $0<\alpha \leqslant \alpha_{0}$ and $1<\beta \leqslant \beta_{0}$ the family $\mathcal{F}_{d}=\left\{\varphi_{\mathbf{x}_{j, k}}\right\}_{j \in \mathbb{N}_{0}, k \in \mathbb{Z}^{d}}$ with $\mathbf{x}_{j, k}=\left(\alpha k \beta^{-j}, \beta^{-j}\right)$ for $j \in \mathbb{N}$ and $\mathbf{x}_{0, k}=(\alpha k, \infty)$ is a discrete wavelet frame with a corresponding dual frame $\mathcal{E}_{d}=\left\{e_{j, k}\right\}_{j \in \mathbb{N}_{0}, k \in \mathbb{Z}^{d}}$ such that

(a) If $f \in F_{p(\cdot), q(\cdot)}^{w}\left(\mathbb{R}^{d}\right)$ we have the quasi-norm equivalence

$$
\begin{aligned}
\left\|f \mid F_{p(\cdot), q(\cdot)}^{w}\left(\mathbb{R}^{d}\right)\right\| & =\left\|\left\{\left\langle f, \varphi_{x_{j, k}}\right\rangle\right\}_{j \in \mathbb{N}_{0}, k \in \mathbb{Z}^{d}} \mid\left(P_{p(\cdot), q(\cdot), a}^{\tilde{w}}\right)^{b}\right\| \\
& \simeq\left\|\left\{\left\langle f, e_{j, k}\right\rangle\right\}_{j \in \mathbb{N}_{0}, k \in \mathbb{Z}^{d}} \mid\left(P_{p(\cdot), q(\cdot), a}^{\tilde{w}}\right)^{\natural}\right\| .
\end{aligned}
$$

(b) For every $f \in F_{p(\cdot), q(\cdot)}^{w}\left(\mathbb{R}^{d}\right)$ the series $f=\sum_{j \in \mathbb{N}_{0}} \sum_{k \in \mathbb{Z}^{d}}\left\langle f, e_{j, k}\right\rangle \varphi_{x_{j, k}}=\sum_{j \in \mathbb{N}_{0}} \sum_{k \in \mathbb{Z}^{d}}\left\langle f, \varphi_{x_{j, k}}\right\rangle e_{j, k}$ converge unconditionally in the quasi-norm of $F_{p(\cdot), q(\cdot)}^{w}\left(\mathbb{R}^{d}\right)$.

Proof. The assertion is a consequence of the representation $F_{p(\cdot), q(\cdot)}^{w}\left(\mathbb{R}^{d}\right)=\operatorname{Co}\left(\mathcal{F}, P_{p(\cdot), q(\cdot), a}^{\tilde{w}}\right)$ and Theorem 2.48. In fact, Proposition 4.6 proves that $\mathcal{F}$ has property $D(\delta, \nu, Y)$ and $D\left(\delta, \nu, L_{2}\right)$ for every $\delta>0$. Also note that $\left(P_{p(\cdot), q(\cdot), a}^{w}\right)^{b}=\left(P_{p(\cdot), q(\cdot), a}^{w}\right)^{\natural}$ with equivalent quasi-norms. 


\subsection{Wavelet bases}

According to Appendix A.2 we obtain a family of systems $\mathcal{G}_{c}, c \in E:=\{0,1\}^{d}$, whose union constitutes a tensor wavelet system in $L_{2}\left(\mathbb{R}^{d}\right)$. Our aim is now to apply the abstract result in Theorem 2.50 to achieve wavelet basis characterizations of $B_{p(\cdot), \tilde{q}}^{w}\left(\mathbb{R}^{d}\right)$ and $F_{p(\cdot), q(\cdot)}^{w}\left(\mathbb{R}^{d}\right)$. We have to consider the Gramian cross kernels $K_{c}=K_{\mathcal{U}}\left[\mathcal{G}_{c}, \mathcal{F}\right]$ and $K_{c}^{*}=K_{\mathcal{U}}^{*}\left[\mathcal{G}_{c}, \mathcal{F}\right]$ from (2.13) in our concrete setup.

Lemma 4.8. Let $Y=L_{p(\cdot), \tilde{q}, a}^{w}(X)$ or $Y=P_{p(\cdot), q(\cdot), a}^{w}(X)$ with associated weight $\nu=\nu_{w, p(\cdot), q(\cdot)}$ given in (4.2). Assume that $a>0$ and $p(\cdot), q(\cdot), \tilde{q}, w$ fulfill the standing assumptions (4.3). Let further $\mathcal{F}=\mathcal{F}\left(\Phi_{0}, \Phi\right)$ be an admissible wavelet frame, $\mathcal{G}_{c}$ be the systems from above, and $K_{c}=K_{\mathcal{U}}\left[\mathcal{G}_{c}, \mathcal{F}\right], K_{c}^{*}=K_{\mathcal{U}}^{*}\left[\mathcal{G}_{c}, \mathcal{F}\right], c \in E$, the corresponding Gramian cross kernels. Then the kernels $K_{c}$ and $K_{c}^{*}$ define bounded operators from $Y$ to $Y$.

Proof. The proof is analogous to the treatment of the kernels osc in Proposition 4.6, see also [50, Lem. 4.24].

Now we are ready for the discretization of $B_{p(\cdot), \tilde{q}^{w}}^{w}\left(\mathbb{R}^{d}\right)$ and $F_{p(\cdot), q(\cdot)}^{w}\left(\mathbb{R}^{d}\right)$ in terms of orthonormal wavelet bases. We again only state the result for $F_{p(\cdot), q(\cdot)}^{w}\left(\mathbb{R}^{d}\right)$ for the sake of brevity.

Theorem 4.9. Let $p(\cdot), q(\cdot), w \in \mathcal{W}_{\alpha_{1}, \alpha_{2}}^{\alpha_{3}}$ fulfill the standing assumptions (4.3), assume further $a>\max \left\{d / p^{-}, d / q^{-}\right\}+\alpha_{3}$ and let $\tilde{w}$ be given as in (4.4). Let $\psi^{0}, \psi^{1} \in L_{2}(\mathbb{R})$ be the Meyer scaling function and associated wavelet. Then every $f \in F_{p(\cdot), q(\cdot)}^{w}\left(\mathbb{R}^{d}\right)$ has the decomposition

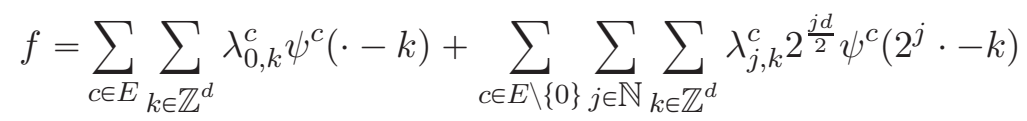

with quasi-norm convergence in $F_{p(\cdot), q(\cdot)}^{w}\left(\mathbb{R}^{d}\right)$ and sequences $\lambda^{c}=\left\{\lambda_{j, k}^{c}\right\}_{j \in \mathbb{N}_{0}, k \in \mathbb{Z}^{d}}$ defined by

$$
\lambda_{j, k}^{c}=\left\langle f, 2^{\frac{j d}{2}} \psi^{c}\left(2^{j} \cdot-k\right)\right\rangle_{\mathcal{S}^{\prime} \times \mathcal{S}} \quad, \quad j \in \mathbb{N}_{0}, k \in \mathbb{Z}^{d},
$$

which belong to the sequence space $\left(P_{p(\cdot), q(\cdot), a}^{\tilde{w}}\right)^{\natural}$ for every $c \in E$. Conversely, an element $f \in$ $\left(\mathcal{H}_{\nu_{\tilde{w}, p(\cdot), q(\cdot)}^{1}}\right)^{\urcorner}$belongs to $F_{p(\cdot), q(\cdot)}^{w}\left(\mathbb{R}^{d}\right)$ if all sequences $\lambda^{c}(f)$ belong to $\left(P_{p(\cdot), q(\cdot), a}^{\tilde{w}}\right)^{\natural}$.

Proof. The statement is a direct consequence of Theorem 4.5 and Theorem 2.50, The required conditions of the kernels $K_{c}, K_{c}^{*}, c \in E$, have been proved in Lemma 4.8 .

\section{A Appendix: Wavelet transforms}

\section{A.1 The continuous wavelet transform}

As usual $\mathcal{S}\left(\mathbb{R}^{d}\right)$ denotes the locally convex space of rapidly decreasing infinitely differentiable functions on $\mathbb{R}^{d}$ and its topological dual is denoted by $\mathcal{S}^{\prime}\left(\mathbb{R}^{d}\right)$. The Fourier transform defined on both $\mathcal{S}\left(\mathbb{R}^{d}\right)$ and $\mathcal{S}^{\prime}\left(\mathbb{R}^{d}\right)$ is given by $\hat{f}(\varphi):=f(\hat{\varphi})$, where $f \in \mathcal{S}^{\prime}\left(\mathbb{R}^{d}\right), \varphi \in \mathcal{S}\left(\mathbb{R}^{d}\right)$, and

$$
\widehat{\varphi}(\xi):=(2 \pi)^{-d / 2} \int_{\mathbb{R}^{d}} e^{-i x \cdot \xi} \varphi(x) d x .
$$

The Fourier transform is a bijection (in both cases) and its inverse is given by $\varphi^{\vee}=\hat{\varphi}(-\cdot)$. 
Let us introduce the continuous wavelet transform. A general reference is provided by the monograph [13, 2.4]. For $x \in \mathbb{R}^{d}$ and $t>0$ we define the unitary dilation and translation operators $\mathcal{D}_{t}^{L_{2}}$ and $T_{x}$ by

$$
\mathcal{D}_{t}^{L_{2}} g=t^{-d / 2} g\left(\frac{\cdot}{t}\right) \quad \text { and } \quad T_{x} g=g(\cdot-x) \quad, \quad g \in L_{2}\left(\mathbb{R}^{d}\right) .
$$

The vector $g$ is said to be the analyzing vector for a function $f \in L_{2}\left(\mathbb{R}^{d}\right)$. The continuous wavelet transform $W_{g} f$ is then defined by

$$
W_{g} f(x, t)=\left\langle T_{x} \mathcal{D}_{t}^{L_{2}} g, f\right\rangle \quad, \quad x \in \mathbb{R}^{d}, t>0,
$$

where the bracket $\langle\cdot, \cdot\rangle$ denotes the inner product in $L_{2}\left(\mathbb{R}^{d}\right)$. We call $g$ an admissible wavelet if

$$
c_{g}:=\int_{\mathbb{R}^{d}} \frac{|\widehat{g}(\xi)|^{2}}{|\xi|^{d}} d \xi<\infty
$$

If this is the case, then the family $\left\{T_{x} \mathcal{D}_{t}^{L_{2}} g\right\}_{t>0, x \in \mathbb{R}^{d}}$ represents a tight continuous frame in $L_{2}(\mathbb{R})$ where $C_{1}=C_{2}=c_{g}$.

Many consideration in this paper are based on decay results of the continuous wavelet transform $W_{g} f(x, t)$. This decay mainly depends on moment conditions of the analyzing vector $g$ as well as on the smoothness of $g$ and the function $f$ to be analyzed, see [59, Lem. A.3] which is based on [52, Lem. 1]

\section{A.2 Orthonormal wavelets}

\section{The Meyer wavelets}

Meyer wavelets were introduced in 44] and are an important example of wavelets which belong to the Schwartz class $\mathcal{S}(\mathbb{R})$. The scaling function $\psi^{0} \in \mathcal{S}(\mathbb{R})$ and the wavelet $\psi^{1} \in \mathcal{S}(\mathbb{R})$ are real, their Fourier transforms are compactly supported and they fulfill

$$
\hat{\psi}^{0}(0)=(2 \pi)^{-1 / 2} \quad \text { and } \quad \operatorname{supp} \hat{\psi}^{1} \subset\left[-\frac{8}{3} \pi,-\frac{2}{3} \pi\right] \cup\left[\frac{2}{3} \pi, \frac{8}{3} \pi\right] .
$$

Due to the support condition we have infinitely many moment conditions (3.6) on $\psi^{1}$ and both functions are fast decaying and infinitly often differentiable, see [60, Section 3.2] for more properties.

\section{Wavelets on $\mathbb{R}^{d}$}

In order to treat function spaces on $\mathbb{R}^{d}$ let us recall the construction of a $d$-variate wavelet basis out of a resolution of unity in $\mathbb{R}^{d}$, see for instance Wojtaszczyk 60. It starts with a scaling function $\psi^{0}$ and a wavelet $\psi^{1}$ belonging to $L_{2}(\mathbb{R})$. For $c \in E=\{0,1\}^{d}$ the function $\psi^{c}: \mathbb{R}^{d} \rightarrow \mathbb{R}$ is then defined by the tensor product $\psi^{c}=\bigotimes_{i=1}^{d} \psi^{c_{i}}$, i.e., $\psi^{c}(x)=\prod_{i=1}^{d} \psi^{c_{i}}\left(x_{i}\right)$, and we let $\mathcal{G}_{c}=\left\{\psi_{(x, t)}^{c}\right\}_{(x, t) \in X}$ be the system with

$$
\psi_{(x, t)}^{c}=\left\{\begin{aligned}
T_{x} \mathcal{D}_{t}^{L_{2}} \psi^{c}, & 0<t<1, \\
T_{x} \psi^{c}, & t=\infty,
\end{aligned} \quad \text { if } c \neq 0 \quad \text { and } \quad \psi_{(x, t)}^{0}=\left\{\begin{aligned}
0, & 0<t<1 \\
T_{x} \psi^{0}, & t=\infty
\end{aligned}\right.\right.
$$




\section{Acknowledgement}

The authors would like to thank Felix Voigtlaender for a careful reading of the manuscript and many valuable comments and corrections. They would further like to thank the anonymous referees for their careful proofreading and many valuable remarks. In particular, Lemma 2.14 should be pointed out, where now - based on their input - also questions of Bochner-measurability are discussed. Furthermore, a serious technical issue in the proof of Theorem 3.11 has been fixed. M.S. would like to thank Holger Rauhut for support during his diploma studies where some ideas of this paper were developed.

\section{References}

[1] S. T. Ali, J.-P. Antoine, and J.-P. Gazeau. Continuous frames in Hilbert space. Ann. Physics, 222(1):1-37, 1993.

[2] A. Almeida and P. Hästö. Besov spaces with variable smoothness and integrability. J. Funct. Anal., 258(5):1628-1655, 2010.

[3] T. Aoki. Locally bounded linear topological spaces. Proc. Imp. Acad. Tokyo, 18:588-594, 1942.

[4] J.-M. Bony. Second Microlocalization and Propagation of Singularities for Semi-Linear Hyperbolic Equations. Taniguchi Symp. HERT. Katata, 11-49, 1984.

[5] H.-Q. Bui, M. Paluszyński, and M. H. Taibleson. A maximal function characterization of weighted Besov-Lipschitz and Triebel-Lizorkin spaces. Stud. Math., 119(3):219-246, 1996.

[6] A. Cohen, I. Daubechies, and J.-C. Feauveau. Biorthogonal bases of compactly supported wavelets. Comm. Pure Appl. Math., 45(5):485-560, 1992.

[7] D. Cruz-Uribe, A. Fiorenza, and C. J. Neugebauer. The maximal function on variable $L^{p}$ spaces. Ann. Acad. Sci. Fenn. Math., 28(1):223-238, 2003.

[8] S. Dahlke, M. Fornasier, H. Rauhut, G. Steidl, and G. Teschke. Generalized coorbit theory, Banach frames, and the relation to alpha-modulation spaces. Proc. London Math. Soc. (3), 96:464-506, 2008.

[9] S. Dahlke, G. Kutyniok, G. Steidl, and G. Teschke. Shearlet coorbit spaces and associated Banach frames. Appl. Comput. Harmon. Anal., 27(2):195-214, 2009.

[10] S. Dahlke, G. Steidl, and G. Teschke. Coorbit spaces and Banach frames on homogeneous spaces with applications to the sphere. Adv. Comput. Math., 21(1-2):147-180, 2004.

[11] S. Dahlke, G. Steidl, and G. Teschke. Weighted coorbit spaces and Banach frames on homogeneous spaces. J. Fourier Anal. Appl., 10(5):507-539, 2004.

[12] S. Dahlke, F. De Mari, E. De Vito, D. Labate, G. Steidl, G. Teschke, S. Vigogna. Coorbit spaces with voice in a Fréchet space. J. Fourier Anal. Appl., DOI 10.1007/s00041-0169466-x. 
[13] I. Daubechies. Ten Lectures on Wavelets, volume 61 of CBMS-NSF Regional Conference Series in Applied Mathematics. Society for Industrial and Applied Mathematics (SIAM), Philadelphia, PA, 1992.

[14] L. Diening. Maximal function on generalized Lebesgue spaces $L^{p(\cdot)}$. Math. Inequal. Appl., $7(2): 245-253,2004$.

[15] L. Diening, P. Harjulehto, P. Hästö, Y. Mizuta, and T. Shimomura. Maximal functions in variable exponent spaces: limiting cases of the exponent. Ann. Acad. Sci. Fenn. Math., 34(2):503-522, 2009.

[16] L. Diening, P. Harjulehto, P. Hästö, and M. Ružička. Lebesgue and Sobolev Spaces with Variable Exponents. Number 2017 in Lecture Notes in Mathematics. Springer, 2011.

[17] L. Diening, P. Hästö, and S. Roudenko. Function spaces of variable smoothness and integrability. J. Funct. Anal., 256(6):1731-1768, 2009.

[18] H. G. Feichtinger. Banach convolution algebras of Wiener's type. In Functions, Series, Operators; Proc. Conf. Budapest 1980, Vol. I, pages 509-524, 1983.

[19] H. G. Feichtinger. Modulation spaces on locally compact Abelian groups. Technical report, January 1983.

[20] H. G. Feichtinger and P. Gröbner. Banach spaces of distributions defined by decomposition methods. I. Math. Nachr., 123:97-120, 1985.

[21] H. G. Feichtinger and K. Gröchenig. A unified approach to atomic decompositions via integrable group representations. In Function spaces and applications (Lund, 1986), volume 1302 of Lecture Notes in Math., pages 52-73. Springer, Berlin, 1988.

[22] H. G. Feichtinger and K. Gröchenig. Banach spaces related to integrable group representations and their atomic decompositions, I. Journ. Funct. Anal., 21:307-340, 1989.

[23] G. B. Folland. Real analysis. Pure and Applied Mathematics (New York). John Wiley \& Sons, Inc., New York, 1984. Modern techniques and their applications, A WileyInterscience Publication.

[24] M. Fornasier and H. Rauhut. Continuous frames, function spaces, and the discretization problem. J. Fourier Anal. Appl., 11(3):245-287, 2005.

[25] P. Balazs and N. Holighaus. Discretization in generalized coorbit spaces: extensions, annotations and errata for " Continuous frames, function spaces, and the discretization problem" by M. Fornasier and H. Rauhut. Online available: https://www . univie.ac.at/nonstatgab/warping/baho15.pdf

[26] P. Balazs, N. Holighaus, and C. Wiesmeyr. Construction of warped time-frequency representations on nonuniform frequency scales, part ii: Integral transforms, functions spaces, atomic decompositions and Banach frames. Online available: arXiv: 1503.05439, 2005.

[27] H. Führ. Coorbit spaces and wavelet coefficient decay over general dilation groups. Trans. Amer. Math. Soc., 367:7373-7401, 2015. 
[28] H. Führ. Vanishing moment conditions for wavelet atoms in higher dimensions. Adv. Comput. Math., 42(1):127-153, 2015.

[29] H. Führ and R. Raisi-Tousi. Simplified vanishing moment criteria for wavelets over general dilation groups, with applications to abelian and shearlet dilation groups. ArXiv e-prints, 2015. arXiv:1407.0824 [math.FA].

[30] H. Führ and F. Voigtlaender. Wavelet coorbit spaces viewed as decomposition spaces. J. Funct. Anal., 269:80-154, 2015.

[31] K. Gröchenig. Unconditional bases in translation and dilation invariant function spaces on $\mathbb{R}^{n}$. In Constructive theory of functions (Varna, 1987), pages 174-183. Publ. House Bulgar. Acad. Sci., Sofia, 1988.

[32] K. Gröchenig. Describing functions: atomic decompositions versus frames. Monatsh. Mathem., 112:1-41, 1991.

[33] K. Gröchenig. Foundations of Time-Frequency Analysis. Appl. Numer. Harmon. Anal. Birkhäuser Boston, 2001.

[34] M. Holschneider. Wavelets, An Analysis Tool. Oxford University Press, 1995.

[35] S. Jaffard, Y. Meyer. Wavelet methods for pointwise regularity and local oscillations of functions. Memoirs of the AMS, vol. 123, 1996.

[36] H. Kempka. 2-microlocal Besov and Triebel-Lizorkin spaces of variable integrability. Rev. Mat. Complut., 22(1):227-251, 2009.

[37] H. Kempka. Atomic, molecular and wavelet decomposition of 2-microlocal Besov and Triebel-Lizorkin spaces with variable integrability. Funct. Appr., 43(2):171-208, 2010.

[38] H. Kempka. Atomic, molecular and wavelet decomposition of generalized 2-microlocal Besov spaces. Journ. Function Spaces Appl., 8:129-165, 2010.

[39] H. Kempka and J. Vybíral. Spaces of variable smoothness and integrability: characterizations by local means and ball means of differences. J. Fourier Anal. Appl., 18(4):852-891, 2012.

[40] H. Kempka and J. Vybíral. A note on the spaces of variable integrability and summability of Almeida and Hästö. Proc. Amer. Math. Soc., 141(9):3207-3212, 2013.

[41] O. Kováčik and J. Rákosník. On spaces $L^{p(x)}$ and $W^{k, p(x)}$. Czechoslovak Math. J., 41(116)(4):592-618, 1991.

[42] Y. Liang, Y. Sawano, T. Ullrich, D. Yang, and W. Yuan. New characterizations of BesovTriebel-Lizorkin-Hausdorff spaces including coorbits and wavelets. J. Fourier Anal. Appl., 18(5):1067-1111, 2012.

[43] Y. Liang, Y. Sawano, T. Ullrich, D. Yang, and W. Yuan. A new framework for generalized Besov-type and Triebel-Lizorkin-type spaces. Diss. Math., 489, 2013.

[44] Y. Meyer. Principe d'incertitude, bases hilbertiennes et algèbres d'opérateurs. Sém. Bourbaki, 28:209-223, 1985-1986. 
[45] A. Nekvinda. Hardy-Littlewood maximal operator on $L^{p(x)}(\mathbb{R})$. Math. Inequal. Appl., $7(2): 255-265,2004$.

[46] W. Orlicz. Über konjugierte Exponentenfolgen. Studia Math., 3:200-212, 1931.

[47] J. Peetre. On spaces of Triebel-Lizorkin type. Ark. Mat., 13:123-130, 1975.

[48] H. Rauhut. Coorbit space theory for quasi-Banach spaces. Studia Math., 180(3):237-253, 2007.

[49] H. Rauhut. Wiener amalgam spaces with respect to quasi-Banach spaces. Colloq. Math., 109(2):345-362, 2007.

[50] H. Rauhut and T. Ullrich. Generalized coorbit space theory and inhomogeneous function spaces of Besov-Lizorkin-Triebel type. J. Funct. Anal., 260(11):3299-3362, 2011.

[51] S. Rolewicz. On a certain class of linear metric spaces. Bull. Acad. Polon. Sci. Cl. III., 5:471-473, XL, 1957.

[52] V. S. Rychkov. On a theorem of Bui, Paluszyński and Taibleson. Proc. Steklov Inst., 227:280-292, 1999.

[53] M. Schäfer. Generalized coorbit space theory for quasi-banach spaces. Diplomarbeit, Rheinische Friedrich-Wilhelms-Universität Bonn, Germany, 2012.

[54] F. Voigtlaender. Embedding theorems for decomposition spaces with applications to wavelet coorbit spaces. PhD thesis, RWTH Aachen University, Germany, 2015. Online available: https://publications.rwth-aachen.de/record/564979/files/564979.pdf

[55] H. Triebel. Theory of Function Spaces. Birkhäuser, Basel, 1983.

[56] H. Triebel. Characterizations of Besov-Hardy-Sobolev spaces: a unified approach. Journ. of Approx. Theory, 52:162-203, 1988.

[57] H. Triebel. Theory of Function Spaces II. Birkhäuser, Basel, 1992.

[58] A. I. Tyulenev. Some new function spaces of variable smoothness. Mat. Sb., 206(6): 85-128, 2015.

[59] T. Ullrich. Continuous characterizations of Besov-Lizorkin-Triebel spaces and new interpretations as coorbits. Journ. Funct. Spaces Appl., Article ID 163213, 2012.

[60] P. Wojtaszczyk. A Mathematical Introduction to Wavelets. Cambridge University Press, 1997. 